\title{
DEVELOPMENT AND CHARACTERIZATION OF TISSUE ENGINEERED BLOOD VESSEL MIMICS UNDER "DIABETIC" CONDITIONS
}

\author{
A Thesis \\ presented to \\ the Faculty of California Polytechnic State University, \\ San Luis Obispo
}

In Partial Fulfillment of the Requirements for the Degree Master of Science in Biomedical Engineering

by

Shelby Gabrielle Kunz

June 2017 
(C) 2017

Shelby Gabrielle Kunz

\section{ALL RIGHTS RESERVED}




\section{COMMITTEE MEMBERSHIP}

TITLE: Development and Characterization of Tissue

Engineered Blood Vessel Mimics Under

"Diabetic" Conditions

AUTHOR: $\quad$ Shelby Gabrielle Kunz

DATE SUBMITTED: June 2017

COMMITTEE CHAIR: Kristen O’Halloran Cardinal, Ph. D.

Associate Professor of Biomedical Engineering

COMMITTEE MEMBER: Trevor Ryan Cardinal, Ph. D.

Associate Professor of Biomedical Engineering

COMMITTEE MEMBER: Jo Ellen Hose, Ph. D.

Lecturer in Biomedical Engineering 


\begin{abstract}
Development and Characterization of Tissue Engineered Blood Vessel Mimics Under "Diabetic" Conditions Shelby Gabrielle Kunz
\end{abstract}

The development of tissue engineered blood vessel mimics for the testing of intravascular devices in vitro has been established in the Cal Poly tissue engineering lab. Due to the prevalence of cardiovascular disease in diabetic patients and minimal accessible studies regarding the interactions between diabetes and intravascular devices used to treat vascular disease, there is a need for the development of diabetic models that more accurately represents diabetic processes occurring in the blood vessels, primarily endothelial dysfunction. This thesis aimed to create a diabetic blood vessel mimic by implementing a high glucose environment for culturing human endothelial cells from healthy umbilical veins (HUVECs) and from diabetic coronary arteries (DHCAECs). The characterization of these BVMs was achieved using immunofluorescence, scanning electron microscopy (SEM), and qPCR gene expression analysis.

From this study, it was determined that HUVECs and DHCAECs are robust enough to be cultured in a high glucose environment - analogous to hyperglycemia - and these cells exhibited different characteristics when evaluated under microscopy and qPCR gene expression. The immunofluorescence and SEM imaging showed presence of cells within each blood vessel mimic. The qPCR gene expression analysis demonstrated that mRNA expression of endothelial nitric oxide synthase (eNOS), platelet endothelial cell adhesion molecule (PECAM), and receptor for advanced glycation end products (RAGE) differs between HUVECs and DHCAECs, as well as between cells cultured in 
normal and elevated glucose concentrations. These differences in gene regulation indicate the potential of the diabetic BVM to more accurately represent the endothelial response to diabetes and to the implementation of intravascular devices in the future. It was determined that culturing DHCAECs in a high glucose cell media for use in blood vessel mimics results in a model that differs considerably from HUVECs grown in normal glucose media. It was also determined that there was a difference between DHCAECs cultured in high glucose media and normal glucose media, as well as HUVECs cultured in high glucose media and normal glucose media. This study aided the development of a diabetic BVM; however, there are still improvements to be made, namely the inclusion of vascular smooth muscle cells in the model and improving the confluency of the BVM. 


\section{ACKNOWLEDGMENTS}

First, I would like to thank Dr. Kristen Cardinal for her guidance and support of my endeavor to achieve a Master of Science degree; without her unending care and compassion for her students - especially those involved in her research laboratory - this thesis project would not have been possible.

To the members of my committee, thank you for your interest in this project and for your patience and expertise throughout this process. I would also like to thank my fellow research laboratory members, especially Zach Cutts, Ella Sugerman, Conor Hedigan, and Kristen Temnyk for their assistance in the laboratory, wealth of knowledge, and moral support.

Most importantly, to my parents and my sister, thank you for your limitless patience, unending love and support. 


\section{TABLE OF CONTENTS}

Page

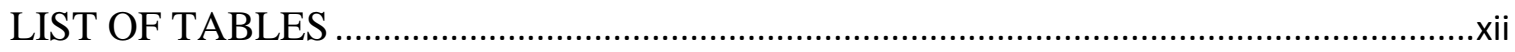

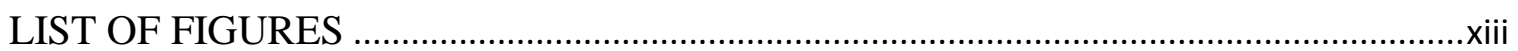

\section{CHAPTER}

1. Introduction to Diabetes and Tissue Engineered Blood Vessel Mimics ........................... 1

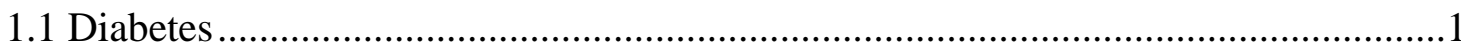

1.1.1 Endothelial Dysfunction in Diabetes Mellitus......................................................

1.1.2 Coronary Artery Disease and Diabetes ........................................................

1.1.3 Efficacy of Coronary Artery Stents ...........................................................

1.2 Tissue Engineered Blood Vessel Mimics...................................................................10

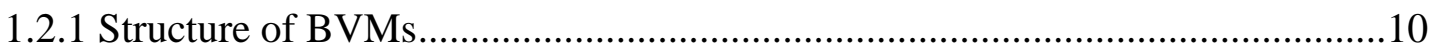

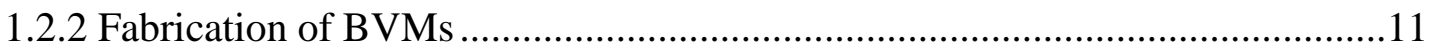

1.3 Development of a Diabetic BVM Model .............................................................12

1.3.1 Background on Immunofluorescence Analysis of BVMs ..................................14

1.3.2 Background on qPCR Analysis of BVMs ....................................................15

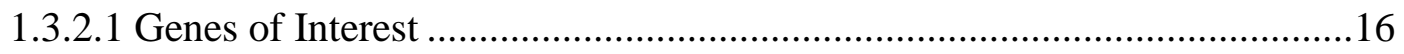

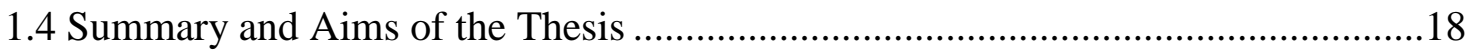

1.4.1 Establishing qPCR Methods for BVM Analysis ..............................................18

1.4.2 Establishing Glucose Concentrations to Model Hyperglycemia ........................19

1.4.3 Applying High Glucose Concentrations to Diabetic BVMs .............................20 
2. Preliminary Study to Establish Methods for qPCR Analysis of HUVECs and DHCAECs

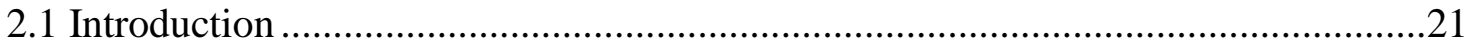

2.2 Principles of Real-Time qPCR ..................................................................21

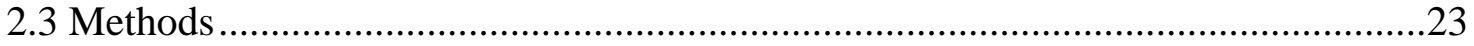

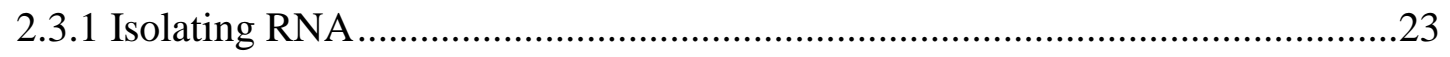

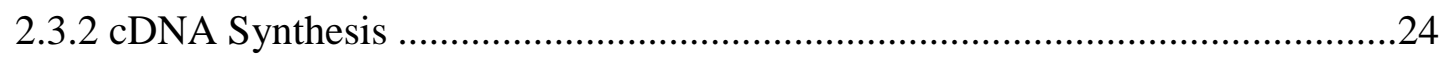

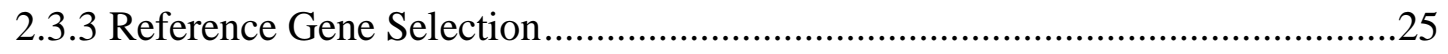

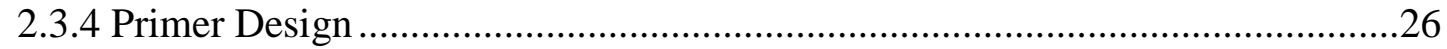

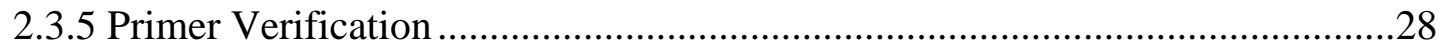

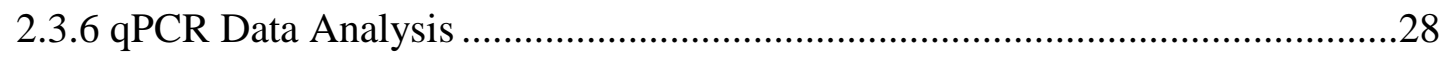

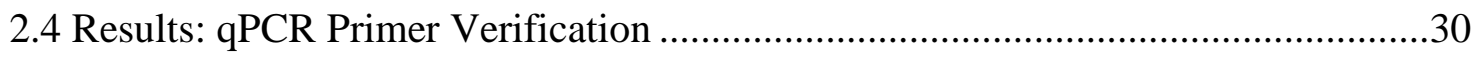

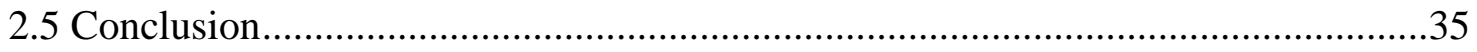

3. Preliminary Study to Develop a Hyperglycemic Condition In Vitro............................... 36

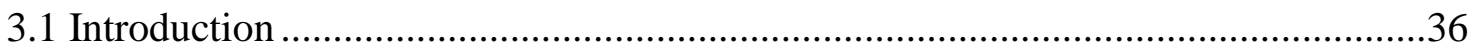

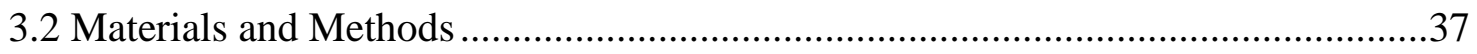

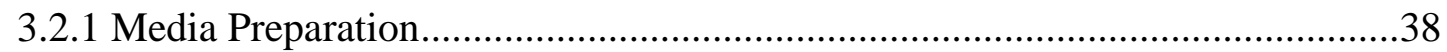

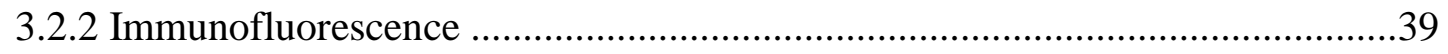

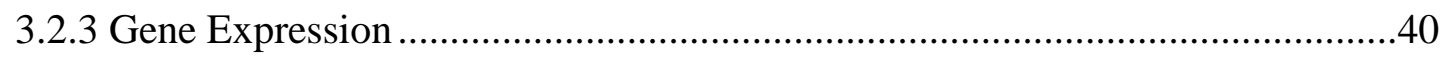

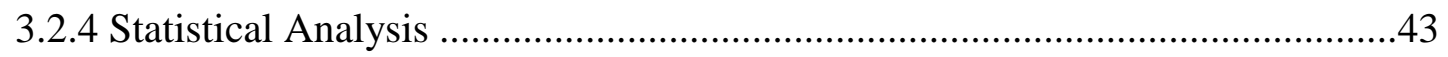


3.3.1 Observations of Culture

3.3.2 Immunofluorescence

3.3.3 Gene Expression .45

3.3.3.1 Comparisons Between Glucose Concentrations

3.3.3.2 Comparisons Between Cell Type

3.3.3.3 Reference Gene Selection

3.4 Discussion

3.5 Conclusion

4. Applying High Glucose Conditions to HUVECs and DHCAECs in Tissue Engineered Blood Vessel Mimics

4.1 Introduction

4.2 Materials and Methods .62

4.2.1 BVM Set Up .62

4.2.2 Take Down and Evaluation .63

4.2.3 Statistical Analysis . .65

4.3 Results .65

4.3.1 Immunofluorescence .65

4.3.2 SEM Imaging...... 69

4.3.3 Gene Expression. .70 


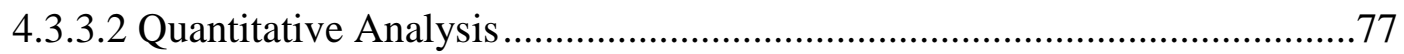

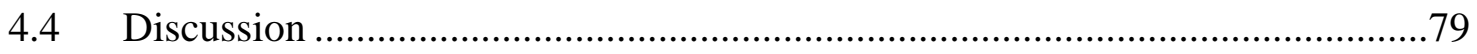

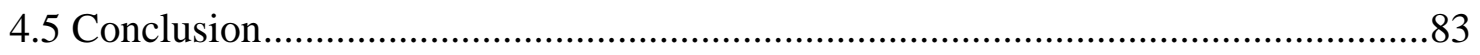

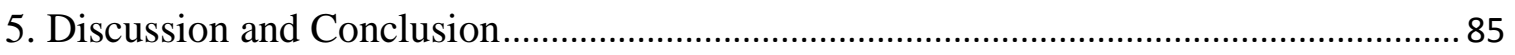

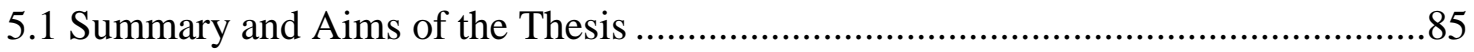

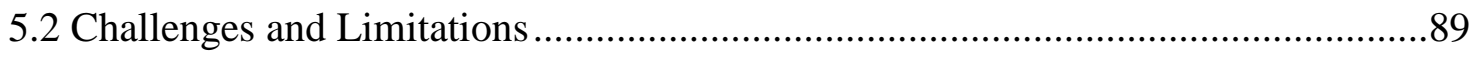

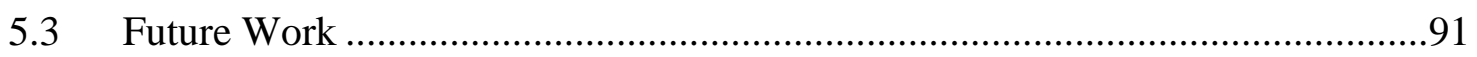

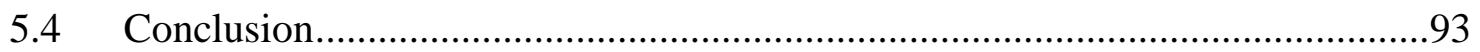

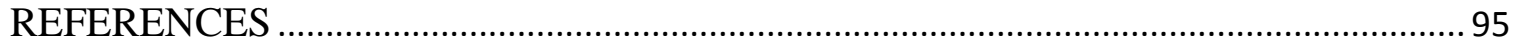

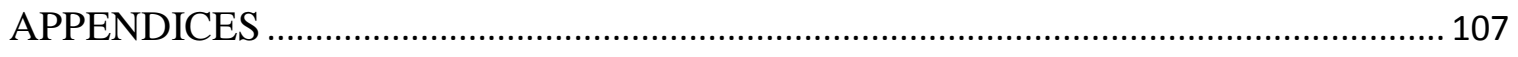

A. Immunofluorescence Staining Protocol for PECAM …….......................................107

B. Protocol for RNA Isolation from Cultured Cells and BVM Samples (SOP7100) ..110

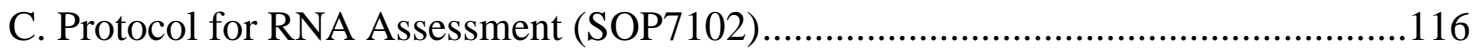

D. Protocol for Reverse Transcription (SOP7103) ……..........................................119

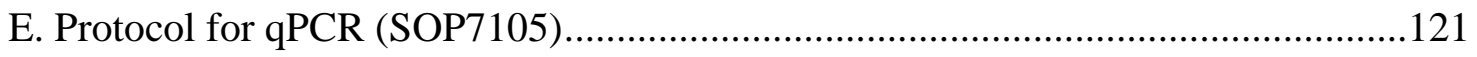

F. Protocol for qPCR Data Analysis (SOP7106) ……….........................................125

G. Protocol for Primer Design (SOP7104)...............................................................130

H. Primer Sets Selected for Verification and Subsequent Amplification and

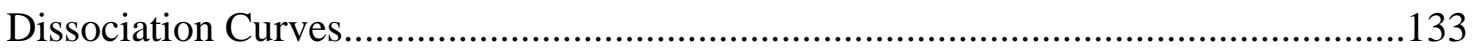

I. Preliminary Study RNA Assessment and Calculations ...........................................134 


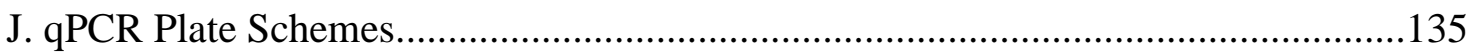

K. Data and Minitab Statistical Analysis from Preliminary Study ............................137 


\section{LIST OF TABLES}

Table

Page

1. Major complications of diabetes, resulting conditions, and associated outcomes $[3,4$,

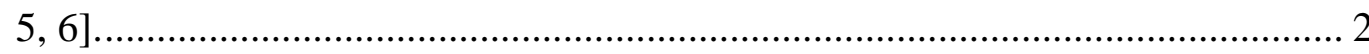

2. Primer sets and sequences for each gene of interest and reference genes. .................. 35

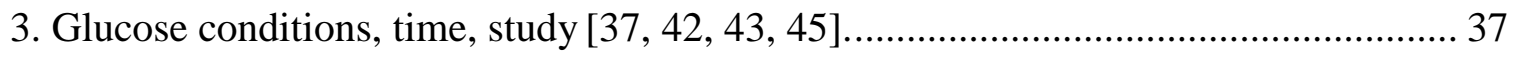

4. Treatment groups to determine glucose concentration. ........................................ 38

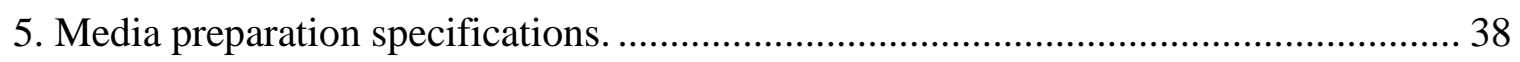

6. Settings used to obtain immunofluorescence images. .......................................... 40

7. Thermocycling settings for 7500 Real-Time PCR System, cycled 40 times............... 42

8. Primer sets and characteristics for primer verification study.............................. 133

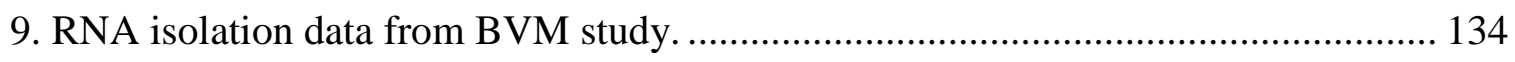




\section{LIST OF FIGURES}

Figure

1. Processes initiated by hyperglycemia that contribute to endothelial dysfunction [8].... 4

2. (A) Effects of increased glucose on the polyol pathway [10]. (B) Glucose oxidation reaction resulting in the formation of $\mathrm{NADH}$

3. Progression of endothelial dysfunction into atherosclerosis and coronary artery disease [13] 7

4. (A) Anatomical cross-section of artery [27]. (B) Theoretical cross-sectional structure of tissue engineered blood vessel mimic. 11

5. Blood vessel mimic bioreactor set up in incubator at $37^{\circ} \mathrm{C}$ and $5 \% \mathrm{CO}_{2}$.

6. Progression of processes involved in the development of atherosclerosis from hyperglycemia. 17

7. Operating principle of polymerase chain reaction [47]. 23

8. RNA purification using spin column methods. Source: www.thermofisher.com. 24

9. 96-well plate template, with each color representing different primer, for primer verification.

10. Amplification curves from primer verification for two different primer sets for

GAPDH.

11. Dissociation curves from primer verification for two different primer sets of

GAPDH.

12. Sample and treatment scheme for immunofluorescence analysis

13. Example of 96-well template used to load samples, where each row represents a different sample group apart from row $\mathrm{H}$ : non-template controls. 
14. Immunofluorescence staining of HUVECs at 10x objective magnification. 44

15. Immunofluorescence staining of DHCAECs at 10x objective magnification. 45

16. Amplification curve for GAPDH from 7500 Real-Time qPCR System. 46

17. Dissociation curve for GAPDH from 7500 Real-Time qPCR System. 47

18. Amplification curve for PECAM from 7500 Real-Time qPCR System................... 47

19. Dissociation curve for PECAM from 7500 Real-Time qPCR System..................... 48

20. Amplification curve for RAGE from 7500 Real-Time qPCR System. .................... 49

21. Dissociation curve for RAGE from 7500 Real-Time qPCR System....................... 49

22. Amplification curve for eNOS from 7500 Real-Time qPCR System....................... 50

23. Dissociation curve for eNOS from 7500 Real-Time qPCR System........................ 50

24. Results of average relative abundance of PECAM in mRNA isolated from

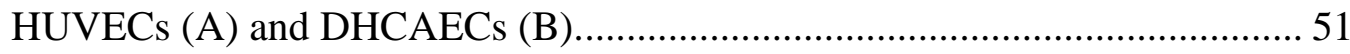

25. Results of average relative abundance of RAGE in mRNA isolated from HUVECs (A) and DHCAECs (B) 52

26. Results of average relative abundance of eNOS in mRNA isolated from HUVECs (A) and DHCAECs (B) 52

27. Results of relative abundance of PECAM in mRNA for normal glucose (A), moderate glucose (B), and high glucose (C) concentrations. 53

28. Results of relative abundance of RAGE in mRNA for normal glucose (A), moderate glucose (B), and high glucose (C) concentrations. 54

29. Results of relative abundance of eNOS in mRNA for normal glucose (A), moderate glucose (B), and high glucose (C) concentrations. 54 
30. BVMs cultivated with HUVECs in control (A-C) and $25.5 \mathrm{mM}$ of glucose (D-F). (A, D) BBI staining of BVM cross-sections. (B, E) PECAM staining of BVM cross-sections. (C, F) Composite image of both immunofluorescence stains.

31. BVMs cultivated with DHCAECs in control (A-C) and 25.5mM of glucose (D-F). (A, D) BBI staining of BVM cross-sections. (B, E) PECAM staining of BVM cross-sections. (C, F) Composite image of both immunofluorescence stains. 68

32. SEM images obtained at 50x magnification of BVMs cultured with HUVECs in control media (A), HUVECs in high glucose media (B), DHCAECs in control media (C), and DHCAECs in high glucose media (D). 69

33. SEM images obtained at 500x magnification of BVMs cultured with DHCAECs in control media (A) and DHCAECs in high glucose media (B). 70

34. Amplification curves for plate 1 (A) and plate 2 (B) for GAPDH. ............................ 71

35. Dissociation curves for plate 1 (A) and plate 2 (B) for GAPDH................................ 72

36. Amplification curves for plate 1 (A) and plate 2 (B) for PECAM............................ 73

37. Dissociation curves for plate 1 (A) and plate 2 (B) for PECAM............................... 73

38. Amplification curves for plate 1 (A) and plate 2 (B) for RAGE. ............................... 74

39. Dissociation curves for plate 1 (A) and plate 2 (B) for RAGE.................................. 75

40. Amplification curves for plate 1 (A) and plate 2 (B) for eNOS. .............................. 76

41. Dissociation curves for plate 1 (A) and plate 2 (B) for eNOS................................... 76

42. Relative abundance of PECAM for each treatment group.......................................... 77 
43. Relative abundance of RAGE for each treatment group, where $* p<0.05$ and $* * \mathrm{p}<0.01$

44. Relative abundance of eNOS for each treatment group, where $* p<0.05$.

45. First plate scheme for experiment in chapter 4, where each color represents a gene:

GAPDH, PECAM, RAGE, and eNOS; rows C and D are RNA samples isolated from BVMs composed of DHCAECs and cultured in normal glucose media; rows E-G are RNA samples isolated from BVMs composed of DHCAECs and cultured in high glucose media; and row $\mathrm{H}$ are non-template controls 135

46. Second plate scheme for experiment in chapter 4 , where each color represents a gene: GAPDH, PECAM, RAGE, and eNOS; rows A and B are RNA samples from BVMs composed of HUVECs and cultured in normal glucose media; rows $\mathrm{C}-\mathrm{F}$ are RNA samples from BVMs composed of HUVECs and cultured in high glucose media; and row are non-template controls 


\section{Chapter 1: Introduction to Diabetes and Tissue Engineered Blood Vessel Mimics}

\subsection{Diabetes}

According to the Centers for Disease Control and Prevention in 2014, 29.1 million people in the United States 20 years or older have diabetes, accounting for approximately $9.3 \%$ of the total population [1]. Diabetes is considered a major epidemic of this century [2]. This disease affects people directly and indirectly, altering their lives significantly from the invasiveness of medical treatment to the accompanying financial consequences. In 2012, it is estimated that diabetes mellitus costs in the United States reached \$245 billion, which is comprised of direct medical costs, disability, work loss, and premature death [1].

Diabetes mellitus is a chronic metabolic disease that results in hyperglycemia due to insulin insufficiency. The method by which the insulin insufficiency develops indicates type 1 versus type 2 diabetes. In type 1 diabetes mellitus there is autoimmune destruction of beta-cells of the pancreas that produce insulin, resulting in an insulin deficiency. In type 2 diabetes there is reduced insulin production by the beta-cells; however, the patients' cells - primarily of skeletal muscle, liver, and adipose tissue - are insulin resistant resulting in hyperglycemia, hyperinsulinemia, and impaired insulin function [3, 4]. In both type 1 and type 2 diabetes there are significant microvascular and macrovascular complications that arise because of the chronic elevation of blood glucose levels [4]. These complications, illustrated in table 1 , include retinopathy leading to loss of vision; nephropathy leading to renal failure; peripheral neuropathy with risk of foot ulcers, amputations and Charcot joints; and autonomic neuropathy causing gastrointestinal, genitourinary, and cardiovascular symptoms [3]. Diabetic patients are 
also at a higher risk for developing atherosclerotic cardiovascular, peripheral arterial, and cerebrovascular disease [3].

Table 1: Major complications of diabetes, resulting conditions, and associated outcomes $[3,4,5,6]$.

\begin{tabular}{|c|c|c|c|}
\hline \multirow{2}{*}{$\begin{array}{c}\text { Microvascular } \\
\text { Retinopathy }\end{array}$} & \multicolumn{3}{|c|}{ Macrovascular } \\
\hline & Loss of vision & $\begin{array}{l}\text { Cardiovascular } \\
\text { disease }\end{array}$ & $\begin{array}{l}\text { Myocardial } \\
\text { infarction }\end{array}$ \\
\hline Nephropathy & Renal Failure & $\begin{array}{l}\text { Cerebrovascular } \\
\text { disease }\end{array}$ & Stroke \\
\hline $\begin{array}{l}\text { Peripheral } \\
\text { Neuropathy }\end{array}$ & $\begin{array}{l}\text { Foot ulcers, } \\
\text { amputations, and } \\
\text { Charcot joints }\end{array}$ & & \\
\hline \multicolumn{4}{|c|}{ Other } \\
\hline $\begin{array}{l}\text { Autonomic } \\
\text { Neuropathy }\end{array}$ & $\begin{array}{l}\text { Gastrointestinal, and } \\
\text { genitourinary } \\
\text { symptoms (sexual } \\
\text { dysfunction) }\end{array}$ & $\begin{array}{l}\text { Depression } \\
\text { Dementia }\end{array}$ & \\
\hline
\end{tabular}

For this thesis, the focus was on the cardiovascular complications of diabetes mellitus and the mechanisms that cause these complications. Cardiovascular disease (CVD) is one of the most detrimental complications of diabetes, accounting for more than half of the mortality of diabetic patients [7]. The term "cardiovascular disease" encompasses numerous conditions: coronary artery disease, high blood pressure, myocardial infarction, arrhythmia and congestive heart failure, among others. For the diabetic population, it is of particular concern for the premature development of atherosclerosis and increased risk of myocardial infarction (MI). A diabetic's risk of MI is equivalent to the risk of a nondiabetic who has previously suffered an MI [7]. These cardiovascular disorders stem from dyslipidemia, poor control of blood glucose, and elevated blood pressure [4]. 
Furthermore, endothelial dysfunction - primarily linked to chronic hyperglycemia - is an underlying cause of atherogenesis and ultimately leads to atherosclerosis [4].

\subsubsection{Endothelial Dysfunction in Diabetes Mellitus}

Diabetes mellitus, with correlated insulin resistance, causes numerous endothelial dysfunctions that compromise the anti-atherogenic role of the vascular endothelium [8]. Hyperglycemia in diabetics contributes to dysfunction of the endothelium by disrupting vascular homeostasis via vasoactive factors - such as angiotensin and nitric oxide compromising permeability, adhesiveness, and integrity [4]. This leads to increased oxidative stress and inflammation, causing blood vessels to become pathogenic, proinflammatory and thrombogenic, leading to premature atherosclerosis and further cardiovascular disorders [4].

In a healthy patient, the endothelium functions to prevent platelet and leukocyte adhesion to maintain the balance of profibrinolytic and prothrombic activity. It is hypothesized that this function is disrupted in diabetic patients due to increased oxidative stress on endothelial cells and vascular smooth muscle cells [4]. There are several hypothesized mechanisms by which chronic hyperglycemia may lead to an increase in oxidative stress in endothelial cells, as seen in Figure 1: (1) activation of the polyol pathway, (2) increased formation of advanced glycation end-products (AGEs), (3) redox potential alterations, and (4) stimulation of the diacylglycerol(DAG)-protein kinase $\mathrm{C}(\mathrm{PKC})$ pathway $[8,9]$. 


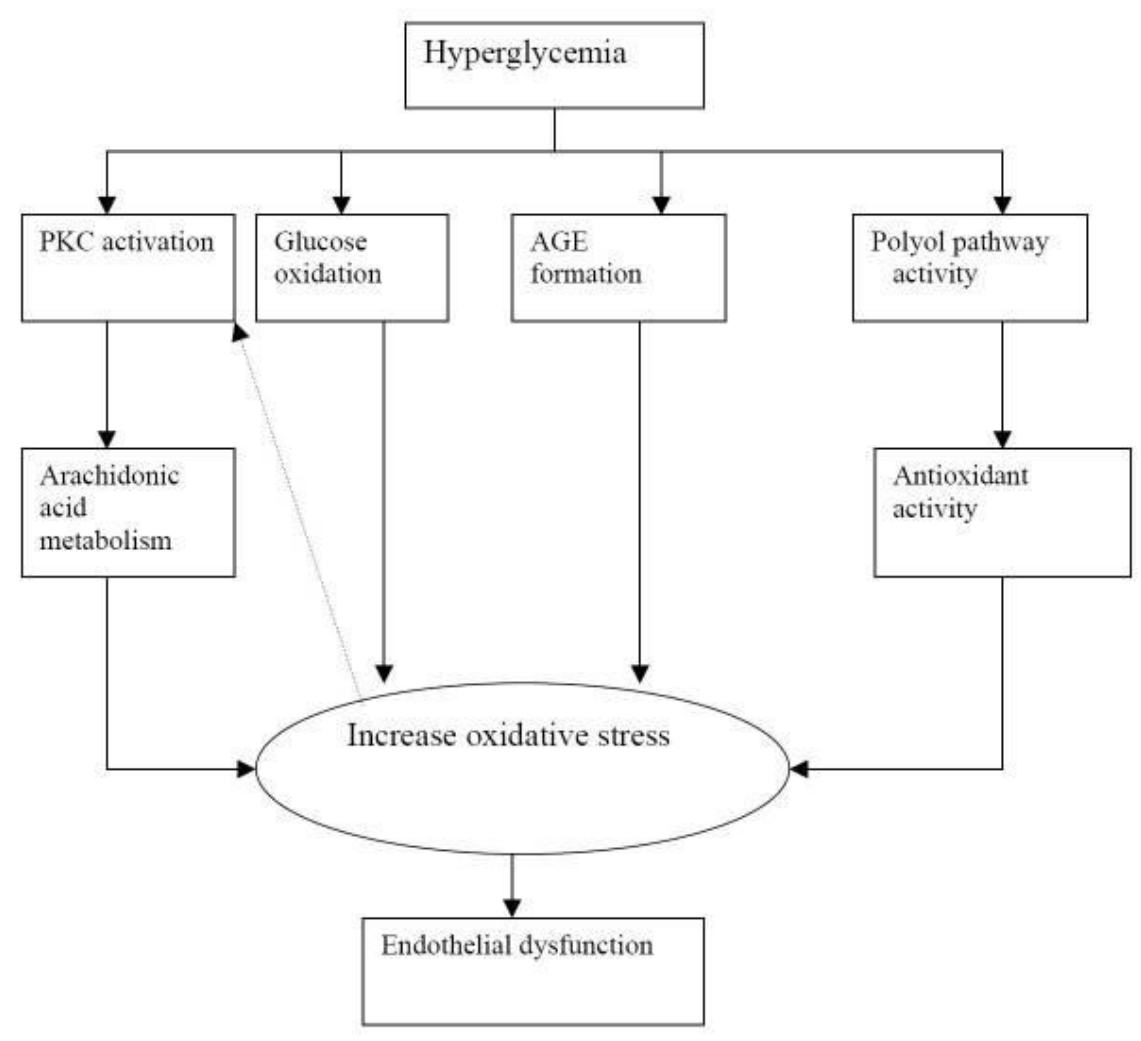

Figure 1: Processes initiated by hyperglycemia that contribute to endothelial dysfunction [8].

The polyol pathway involves the reduction of glucose to sorbitol and the conversion of sorbitol to fructose via aldose reductase (AR) and sorbitol dehydrogenase(SDH), respectively [10]. AR uses reduced nicotinamide adenine dinucleotide phosphate (NADPH) as a co-factor, while SDH uses oxidized nicotinamide adenine dinucleotide (NAD+) as a co-factor [10]. The activation of these enzymes results in the formation of oxidized NADPH, or NADP, and the reduced form of $\mathrm{NAD}^{+}$, or NADH, respectively. The byproduct NADH, of the polyol pathway, is oxidized by NADH oxidase (NOx) to produce reactive oxygen species (ROS) [10]. Fructose, another byproduct of the polyol pathway, is metabolized to fructose-3-phosphate (F-3-P) and 3-deoxyglucosone (3-DG) contribute to advance glycation end-product (AGE) formation, which then binds to its 
receptor to increase oxidative stress [10]. The effects of increased glucose on the polyol pathway is shown in Figure 2A.

Similar to the polyol pathway, glucose oxidation - or the first reaction of glucose metabolism shown in Figure 2B - results in the production of NADH [11]. Increased glucose oxidation in diabetics results in excess NADH - either through glucose metabolism or the polyol pathway - which reacts with the enzyme NADH oxidase to produce ROS [10]. Glucose oxidation also leads to an increase in advanced glycation end-products (AGEs) due to the non-enzymatic reaction of glucose metabolites and other glycation processes.

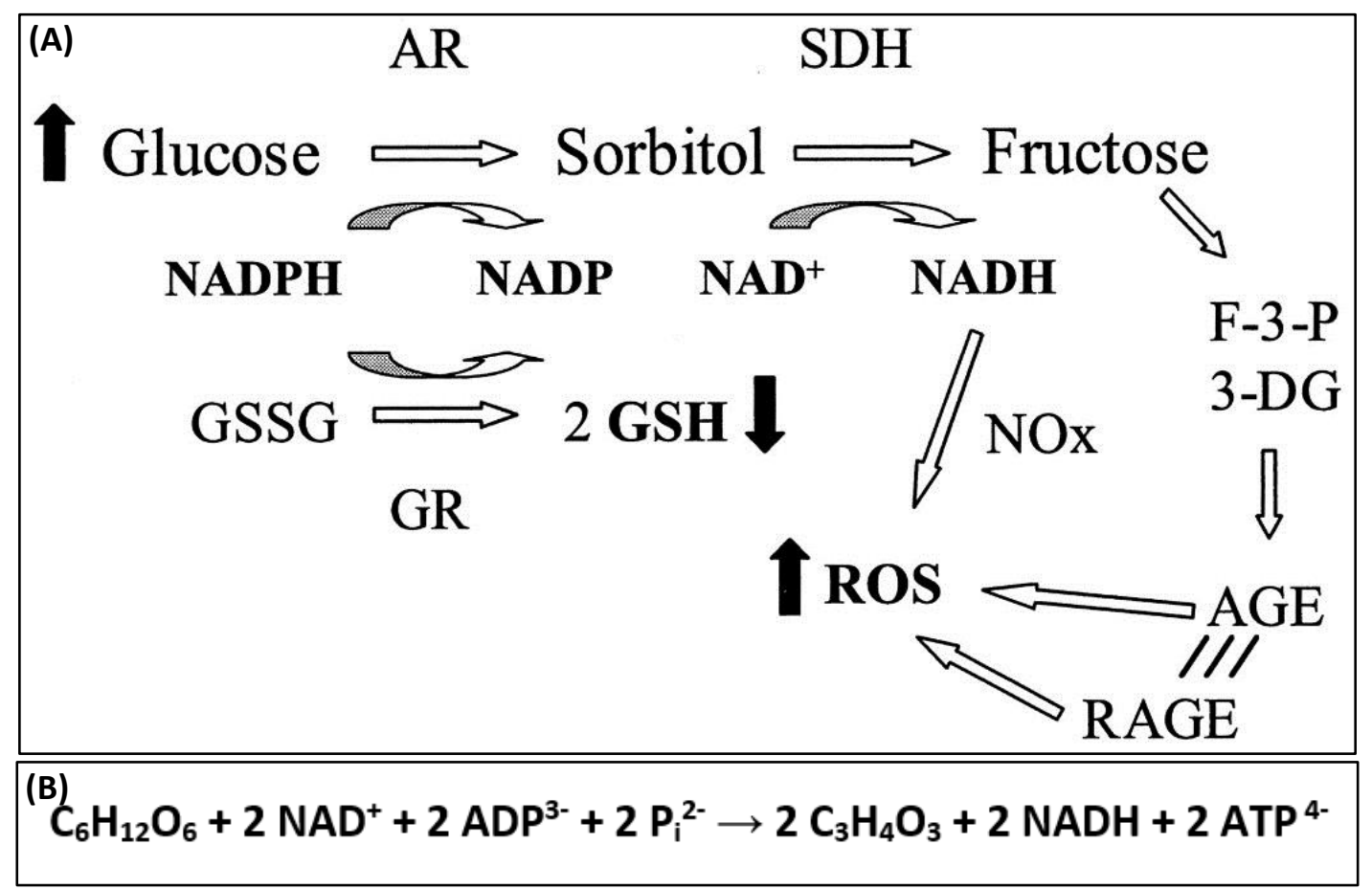

Figure 2: (A) Effects of increased glucose on the polyol pathway [10]. (B) Glucose oxidation reaction resulting in the formation of NADH. 
Hyperglycemia also activates the diacylglycerol (DAG)-protein kinase C (PKC) pathway. Glucose promotes synthesis of DAG, which is crucial in the function of PKC [9]. It is commonly held that PKC may have numerous adverse effects on vascular function through the activation of NADPH oxidase and endothelial nitric oxide synthase (eNOS) [8]. These enzymes produce superoxide $\left(\mathrm{O}_{2}{ }^{-}\right)$which contributes further to oxidative stress and the production of AGEs in the blood vessels of diabetics [8].

The increase in ROS and AGE production contributes to the rapid progression of atherosclerosis seen in diabetes. AGEs accumulate within vessel walls, inhibiting nitric oxide (NO) function on vascular smooth muscle cells (VSMCs), forming collagen crosslinks that alter cell structure and trap plasma proteins, and bind to the receptor for advanced glycation end products (RAGE) to alter cell function [8, 12]. RAGE activation signals transcription of multiple adhesion molecule proteins, increased intake of oxidized low-density lipoproteins (OxLDL) and foam cell formation, as well as NADPH oxidase perpetuating the production of $\operatorname{ROS}[12,13]$. When interacting with the vessel wall, AGEs also initiate the formation of OxLDL, increase vascular permeability, and increase endothelial leukocyte adhesion molecule expression; ultimately resulting in the formation of atherosclerotic plaques within the vessels through processes listed in Figure 3 [8]. 


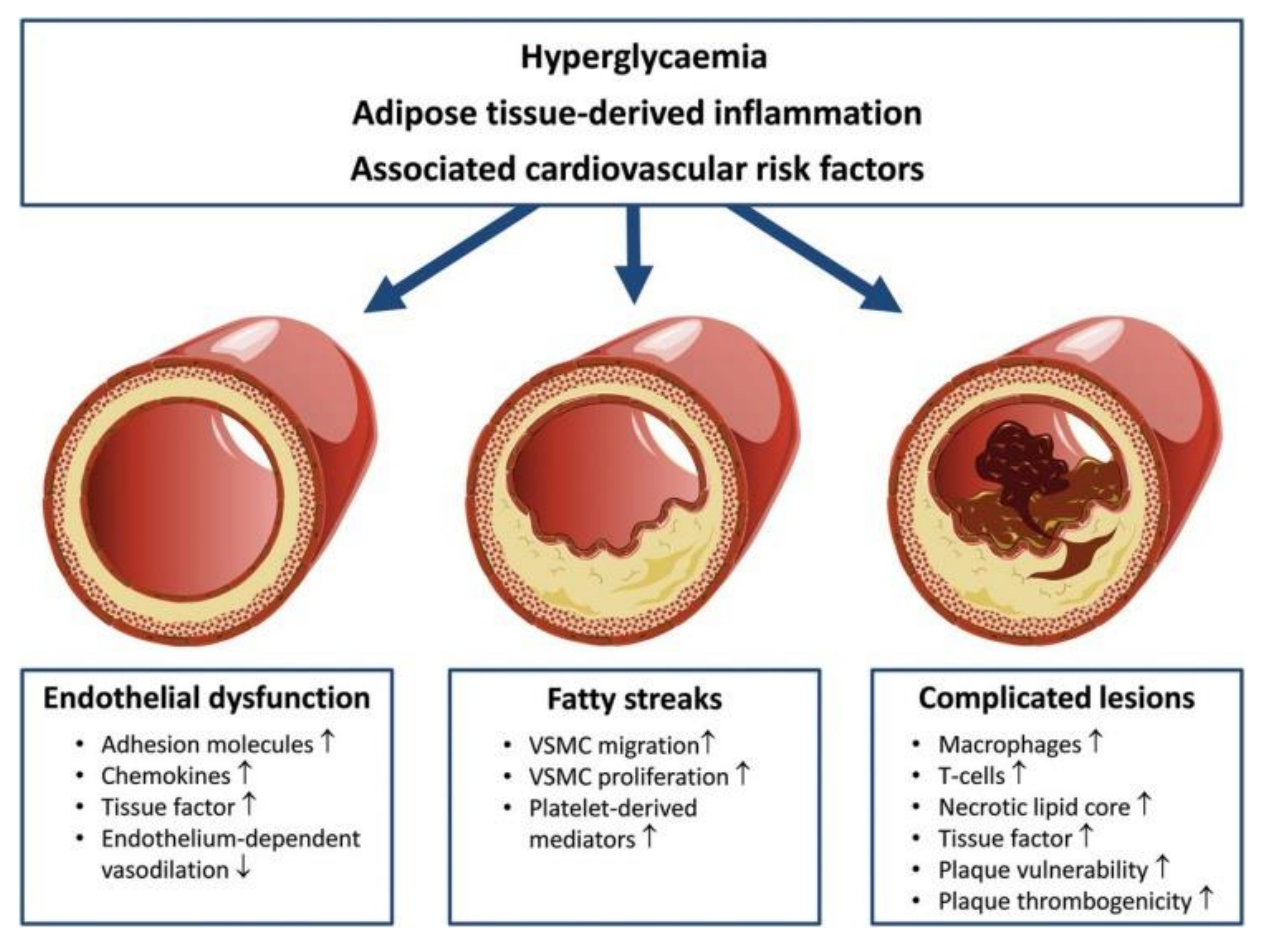

Figure 3: Progression of endothelial dysfunction into atherosclerosis and coronary artery disease [13].

For this thesis, the pathophysiology of diabetes and endothelial dysfunction is important to replicate aspects of this condition in vitro. Although the disease process of diabetes is complex and involves the interaction of numerous cell types, there are some responses that are specific to the endothelium that may be able to be replicated in a diabetic blood vessel model. Ideally, the diabetic blood vessel model will replicate the increase in adhesion molecules, oxidative stress and AGEs - and the respective receptors - which are key in the pathogenesis of endothelial dysfunction associated with diabetes. 


\subsubsection{Coronary Artery Disease and Diabetes}

As diabetes progresses, the effects of endothelial dysfunction cause adults with diabetes to be at an increased risk for coronary artery disease (CAD), heart attack, stroke and

angina [14]. The development of CAD with diabetes is due to endothelial dysfunction, which enhances the formation of atherosclerotic plaques. The current standard of care is either coronary artery bypass grafting $(\mathrm{CABG})$, or percutaneous coronary intervention (PCI) [10]. CABG, which is typically performed as open-heart surgery, requires making a large incision through the chest and sternum, and placing the patient on cardiopulmonary bypass, to allow the surgeon to reroute blood flow around the blocked vessel using a graft. Bypass grafts can be either an autologous vein or artery obtained from a prior harvesting surgery, or a synthetic graft constructed from biomaterials. PCI is the use of catheters, guided by fluoroscopy, to gain access to the affected coronary artery to place a stent that maintains patency of the vessel. This treatment method is often preferred to CABG because is it less invasive and capable of treating complex lesions [15].

\subsubsection{Efficacy of Coronary Artery Stents}

The treatment of CAD in patients with diabetes has been shown to have worse outcomes in terms of mortality, myocardial infarction and need for revascularization than patients without diabetes [16]. In one specific study, diabetics had higher mortality after PCI compared to $\mathrm{CABG}$, which may be attributed to the medical therapies used to control hyperglycemia and other systemic symptoms of diabetes [17]. Another study found that diabetic patients, within a population experiencing ST-segment elevation myocardial infarction (STEMI), had a worse outcome at 3-year follow-up after PCI, compared to the 
STEMI population without diabetes [18]. These researchers also found that diabetic patients were at a higher risk of stent thrombosis; however, this risk was reduced with the advent of everolimus-eluting stents [18]. Everolimus, an immunosuppressant drug, is used to prevent restenosis of vessels after PCI treatment of CAD, and may or may not interact with the pathological mechanisms of diabetes. These investigations are primarily retrospective and observational, such as subgroup analyses within a larger study [16, 18]. This means that there is not a wealth of data available for the evaluation of such therapies for diabetic patients that would allow for the improvement of treatment and, ultimately, patient outcomes. Due to the increased morbidity and mortality of this subgroup of patients, there is a growing need for the development of a well-defined model for further evaluation of CAD treatment -as well as other medical therapies-in diabetic patients [16]. There have been several animal models developed to investigate various aspects of the diabetic condition, including streptozotocin-induced diabetic rats and mice, spontaneously mutated rats such as Zucker Diabetic Fatty Rats (ZDF), genetically modified mice models, diabetic Yucatan miniswine, and streptozotocin-induced diabetic domestic pigs $[19,20]$. However, rodent models are anatomically very small for testing stents or other intravascular devices and all animal models may differ in vascular and endothelial cells outcomes due to species-specific responses [21]. Therefore, to obtain a better understanding of the effectiveness of intravascular devices such as stents in a diabetic environment specific to humans, a tissue engineered blood vessel mimic can be used as a simplified model for preclinical in vitro testing. 


\subsection{Tissue Engineered Blood Vessel Mimics}

In recent years, tissue engineering has emerged as a new scientific field that involves the fabrication of new and functional living tissue from living cells in combination with a matrix or scaffolding to guide development [22]. Within this paradigm of cells and scaffold, there are a myriad of options available depending on the tissue being created. One application for tissue engineering is the creation of small diameter vascular grafts for CABG that might mimic the functional properties of native tissues [23]. Due to the complexity and novelty in the creation of tissue engineered vascular grafts (TEVGs), there have been significant challenges in clinical application [23]. However, the development of TEVGs has led to the employment of these technologies as simple in vitro blood vessel models for preclinical testing of intravascular devices, such as stents [24]. In the Cal Poly Tissue Engineering lab, 3-dimensional tissue-engineered blood vessel constructs - or blood vessel mimics (BVMs) - are being developed as an in vitro preclinical testing environment for intravascular devices [25].

\subsubsection{Structure of BVMs}

As in any tissue engineering application, it is the goal of BVMs to mimic certain characteristics of native blood vessels. Native vessel anatomy features three layers: tunica intima, tunica media, and tunica adventitia as seen in Figure 4A. The tunica intima consists of a monolayer of endothelial cells, or endothelium, that directly interacts with blood, and functions to prevent thrombosis formation and minimize friction for continuous blood flow [26]. The tunica media is composed of smooth muscle cells arranged circularly around the circumference of the vessel with sheets of elastin [26]. 
Finally, the tunica adventitia is the outermost layer and is composed of loosely woven collagen fibers that serve to protect and reinforce the vessel [26].
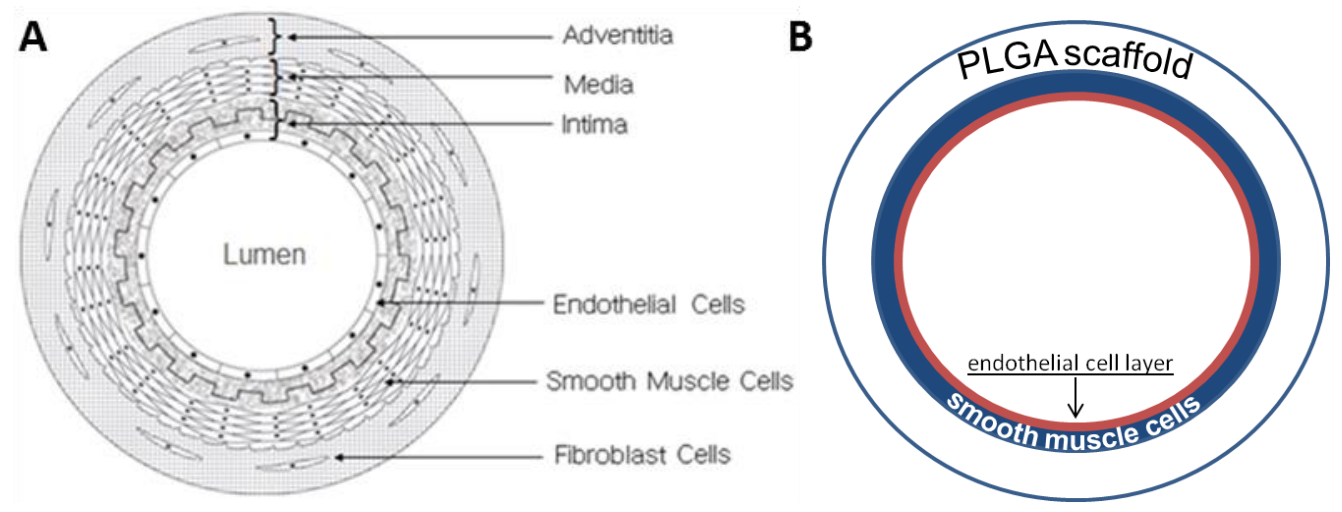

Figure 4: (A) Anatomical cross-section of artery [27]. (B) Theoretical cross-sectional structure of tissue engineered blood vessel mimic.

Currently in the Cal Poly lab, an electrospun poly(lactic-co-glycolic-acid) (PLGA) scaffold is used because of its similarity to the natural collagen and elastin-filled extracellular matrix of blood vessels, as well as its biocompatibility [28]. Primary human smooth muscle cells and endothelial cells, expanded through in vitro culture, are introduced through the lumen of the BVM to replicate the three layers of native vessels, as illustrated in Figure 4B [25].

\subsubsection{Fabrication of BVMs}

A previously established method is used to produce a BVM in the tissue engineering lab [25]. First, the scaffold is conditioned in a high-protein media within a perfusion bioreactor to improve cell adhesion to the synthetic polymer. The bioreactor consists of the chamber containing the BVM, an 8-roller peristaltic pump, and a media reservoir as 
shown in Figure 5. Next, human umbilical artery smooth muscle cells (HUASMCs) are pressure sodded into the lumen of the scaffold, followed by human umbilical vein endothelial cells(HUVECs), in the bioreactor chamber.

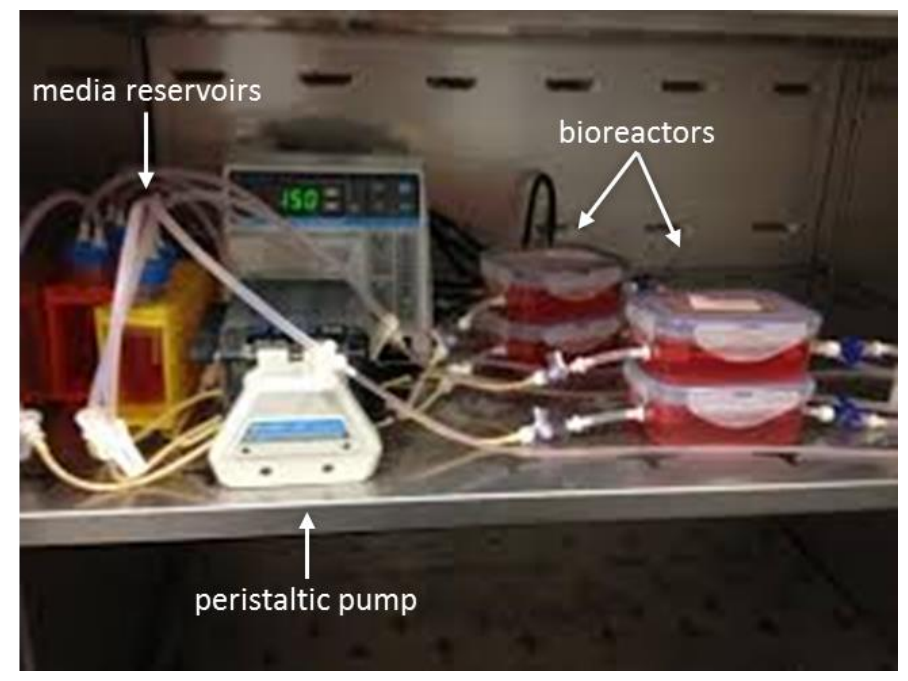

Figure 5: Blood vessel mimic bioreactor set up in incubator at $37^{\circ} \mathrm{C}$ and $5 \% \mathrm{CO}_{2}$.

\subsection{Development of a Diabetic BVM Model}

Currently, the BVMs produced represent a "healthy" vessel using human umbilical vein endothelial cells (HUVECs) and HUASMCs. Other cell sources have been used in BVM construction, including human coronary artery endothelial cells (HCAECs) and to a limited extent, diabetic human coronary artery endothelial cells (DHCAECs) [29]. The initial steps were taken to develop a "diabetic" blood vessel mimic through the development of media solutions of various glucose concentrations to replicate a hyperglycemic environment with bovine aortic endothelial cells (BAECs) and HUVECs [30]. From this study, it was determined that endothelial cells have increased glucose consumption when exposed to increased glucose concentrations in the cell media [30]. 
Although this study provides a foundation for the development of the diabetic blood vessel mimic, it did not incorporate the use of DHCAECs and did not include extensive characterization of the BVMs. The DHCAEC BVM has not been well-characterized and has not been compared to the previously developed healthy BVMs.

For this thesis, potential parameters for a diabetic BVM were studied by comparing the use of HUVECs and DHCAECs in a blood vessel mimic construct, as well as the effects of implementing an in vitro hyperglycemic environment for cell culture. There were two variables in the BVM protocol that were investigated: (1) cell type and (2) concentration of glucose in cell media. Cell media typically contains $5.5 \mathrm{mM}(1 \mathrm{~g} / \mathrm{L})$ of glucose - approximately normal blood glucose levels in vivo - for normal cell function, since glucose is a source of energy [31]. Therefore, the control groups for cell type and glucose concentration were HUVECs and normal endothelial cell media $(5.5 \mathrm{mM}$ glucose), respectively. BVMs for this control group have been well-characterized by previous students using scanning electron microscopy (SEM), immunofluorescence, and histology. Preliminary qPCR analysis has been previously performed on BVMs using HUVECs and human umbilical artery smooth muscle cells (HUASMCs), and HCAECs and human coronary artery smooth muscle cells (HCASMCs); however, the qPCR work was not well-documented and gene expression data is relative to the experimental conditions in question [29]. This means that qPCR methods are not well-established and previous gene expression data cannot be applied in general to BVM characteristics, therefore a brief introduction to these techniques will be provided.

In addition to qPCR characterization, the control and experimental BVMs were also characterized using immunofluorescence, and SEM to verify that the BVMs 
produced were consistent with previous experiments completed by the Cal Poly Tissue Engineering Research Laboratory [25].

\subsubsection{Background on Immunofluorescence Analysis of BVMs}

Immunofluorescence is primarily used to detect the presence of specific cellular proteins via selective antibodies labeled with fluorophores that can be detected under wide field fluorescence, among other imaging and data collecting techniques [32]. This is especially useful in determining phenotype of cells and differentiating between cell types, depending on the cellular proteins chosen to isolate via fluorophore. For tissue engineered blood vessel mimics platelet endothelial cell adhesion molecule (PECAM), also known as CD31, was selected to determine the presence of human endothelial cells in the blood vessel mimic during analysis.

PECAM is a cell junction protein present on endothelial cells that plays a role in cell-to-cell adhesion and the inflammatory process [33]. In situ, PECAM is expressed on continuous endothelium of all blood vessel types and is not expressed on fibroblasts or epithelial cells - which are also present in blood vessels [33, 34]. PECAM has been used as an immunostaining marker for identifying endothelial cells in a variety of tissue engineering applications, including vascular grafts that utilize HUVECs [35, 36, 37, 38].

Due to the prevalence of PECAM at endothelial cell junctions and its specificity to vascular endothelial cells, it is an ideal marker for immunofluorescent staining of HUVECs and DHCAECs in tissue engineered BVMs. 


\subsubsection{Background on qPCR Analysis of BVMs}

Quantitative polymerase chain reaction (qPCR) is a well-established method for analyzing gene expression in biological samples. Gene expression refers to the amount of mRNA present in a sample [39]. qPCR allows for the analysis of specific genes; however, it is not ideal for large quantities of RNA, or samples with complex RNA - such as RNA isolated from multiple cell types - as it has relatively low throughput [39]. For this study, qPCR analysis may be useful in determining gene expression since the focus is on a single cell type for each BVM.

There are several types of qPCR technologies available to accomplish this analysis: endpoint qPCR, real-time qPCR, and reverse-transcription qPCR (RT-qPCR). Endpoint qPCR was one of the first PCR systems that allowed for quantification; however, there are major limitations because of the need to visualize and quantify PCR products after reaction completion through agarose gel electrophoresis [39]. This technique is best used for binary analysis to determine the presence of a gene, rather than the relative expression of a gene [39]. Real-time qPCR allows for the quantification of gene expression throughout the course of the reaction, minimizing experimental error due to reaction efficiency and post-processing [39]. This process uses a fluorescent detector molecule, such as SYBR green, to obtain a quantitative measurement of PCR product in "real time" during each PCR cycle [39]. RT-qPCR is a type of real-time qPCR method that optimizes the protocol by combining the final step of sample preparation with realtime qPCR, minimizing the opportunities for error and contamination. Due to the sensitivity and quantitative measurements obtained by real-time qPCR, this method was used to obtain gene expression data. 


\subsubsection{Genes of Interest}

One of the advantages of qPCR is the ability to determine the expression of a single gene within the entire transcriptome of a given sample; however, this means that the genes selected must be specific to the cell type and disease process of interest. Given that this study is investigating human endothelial cells and diabetes, the genes of interest needed to be selected accordingly.

Platelet Endothelial Cell Adhesion Molecule (PECAM) - As previously mentioned, the effects of diabetes on vascular tissue largely revolves around the onset of endothelial dysfunction due to hyperglycemia. If endothelial dysfunction continues to progress, inflammation of the endothelium occurs and initiates the development of atherosclerosis. PECAM plays a role in endothelial cell interactions, and inflammation processes, and has shown to be expressed in atherosclerotic lesions in vivo [33, 40]. It has been demonstrated that hyperglycemia causes an increase in the expression of adhesion molecules, such as endothelial-leukocyte adhesion molecule-1, vascular cell adhesion molecule 1 (VCAM-1) and intercellular adhesion molecule 1 (ICAM-1) in HUVECs, and inflammation determines the degree of adhesive properties exhibited by human endothelial cells [40]. However, in previous studies, it was found that HUVECs cultured in high glucose conditions in vitro did not have altered PECAM expression compared to HUVECs cultured in normal glucose conditions [41]. This gene was selected to discern whether HUVECs and DHCAECs behave differently when cultured in high glucose conditions, and is associated with increased oxidative stress and endothelial dysfunction due to hyperglycemia, as shown in Figure 6. 


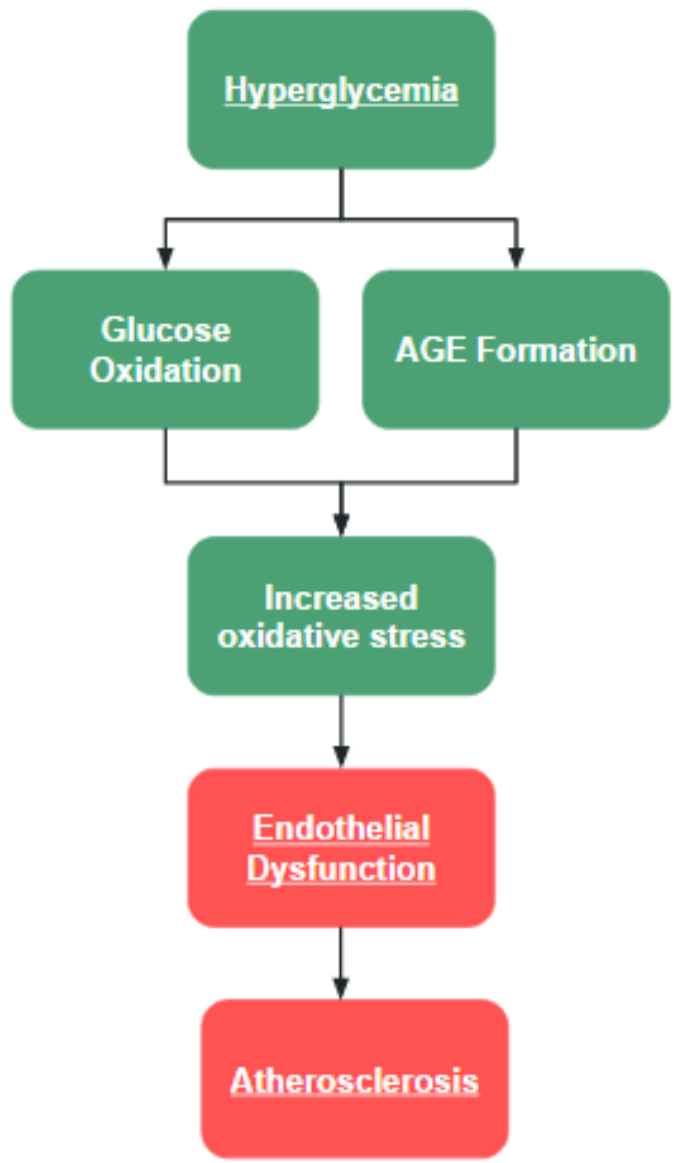

Figure 6: Progression of processes involved in the development of atherosclerosis from hyperglycemia.

Receptor for Advanced Glycation End Products (RAGE) - The production of AGEs plays a large role in the development of endothelial dysfunction, as previously discussed and shown in Figure 6. The increase in AGE production results in increased RAGE activation on endothelial cells, which triggers the transcription factor nuclear factor- $\mathrm{KB}(\mathrm{NF \kappa B})$ that modifies cell function [12]. This change in the biological properties of ECs result in endothelial dysfunction [40]. In studies of vascular inflammation in diabetic patients, it was found that the increase in AGEs due to hyperglycemia results in the increased expression of $R A G E$ in human aortic endothelial 
cells [42]. RAGE was chosen as a gene of interest because it is known to increase in expression when endothelial cells are exposed to elevated glucose conditions, it is also an indicator for the increased production of AGEs, which occurs early in the pathogenesis of diabetic endothelial dysfunction [43].

Endothelial Nitric Oxide Synthase (eNOS or NOSIII) - Endothelial nitric oxide synthase (eNOS) is a protein that produces nitric oxide(NO) found in endothelial cells. The synthesis of NO typically results in vascular smooth muscle relaxation and vasodilation in vivo; however, in diabetes it has been found that NO production is decreased in blood vessels [44]. The direct effect of glucose on NO production has not been well-studied, however it has been suggested that hyperglycemia may alter the function of eNOS, resulting in the production of superoxide $\left(\mathrm{O}_{2}{ }^{-}\right)$rather than $\mathrm{NO}[44,45]$. It has been found that $e N O S$ gene expression in human aortic endothelial cells is increased with prolonged exposure - 5 days - to high glucose [45]. eNOS was chosen as a gene of interest because of its possible involvement with the PKC pathway in the pathogenesis of diabetic endothelial dysfunction that has not been accounted for with PECAM or RAGE.

\subsection{Summary and Aims of the Thesis}

\subsubsection{Establishing qPCR Methods for BVM Analysis}

Currently, BVMs produced in the lab are characterized using hematoxylin and eosin staining, immunofluorescence, and scanning electron microscopy. Although these analyses are beneficial for exhibiting cell presence in the BVMs, they are primarily image-based and do not account for biological processes occurring at the molecular level. 
With the development of a diabetic BVM, the implementation of gene expression analysis would be beneficial to obtain a better understanding of cellular processes in both healthy and diseased cell states, as well as disease processes that may or may not be occurring in the diabetic endothelial cells. The first aim of this thesis was to improve upon previous attempts to complete gene expression analysis on the tissue engineered blood vessel mimics by optimizing and documenting a protocol, and to apply this protocol to the study of HUVECs and DHCAECs in BVMs.

\subsubsection{Establishing Glucose Concentrations to Model Hyperglycemia}

The Tissue Engineering Lab at Cal Poly has successfully developed and characterized a healthy blood vessel mimic using human umbilical vein endothelial cells (HUVECs) and human umbilical artery smooth muscle cells (HUASMCs). These BVMs have been used for the testing of intravascular medical devices in vitro. In recent work by another student, a preliminary "diabetic" BVM was created using diabetic human coronary artery endothelial cells (DHCAECs). Although this preliminary diabetic model demonstrated the ability to create a BVM using DHCAECs, it did not account for the hyperglycemic condition DHCAECs are subjected to in vivo. Therefore, the second aim of this thesis was to improve upon the capabilities for creating "diabetic" tissue engineered blood vessel mimics by determining an appropriate glucose concentration in cell culture media to simulate hyperglycemia, and to distinguish the effects of the simulated hyperglycemic environment on HUVECs and DHCAECs. 


\subsubsection{Applying High Glucose Concentrations to Diabetic BVMs}

Once protocols for implementing qPCR and producing an elevated glucose condition for cell culture were established, the protocols were applied in conjunction with the previously established method for the development of blood vessel mimics. The third, and final, aim of this thesis was to apply and analyze the effects of introducing elevated glucose conditions to BVMs and the use of DHCAECs to more accurately model the hyperglycemic condition of diabetics.

It is hypothesized that the selected genes of interest: PECAM, RAGE, and $e N O S$ will be upregulated, or expression will be increased, in the DHCAECs compared to the HUVECs and in the cells cultivated in high glucose conditions compared to the normal glucose conditions. The increase in the expression of these genes for DHCAECs implemented in a BVM would indicate the potential of the tissue engineered blood vessel mimics to exhibit similar characteristics to diabetic endothelium in blood vessels. 


\section{Chapter 2: Preliminary Study to Establish Methods for qPCR Analysis of HUVECs and DHCAECs}

\subsection{Introduction}

In the tissue engineering lab, there has been previous work to characterize blood vessel mimics (BVMs) using quantitative polymerase chain reaction (qPCR) in order to understand molecular expression of human umbilical vein endothelial cells (HUVECs) and human coronary artery endothelial cells (HCAECs) [29]. However, qPCR analysis has not been repeated or implemented as standard analysis in the lab for current BVM studies. The previous work also did not include the investigation of a diseased cell state; therefore, the genes of interest were not applicable to diabetic cells. The first aim of this thesis is to improve upon the previous qPCR protocol and to include DNA primers for genes of interest associated with the disease process of diabetic endothelial dysfunction.

\subsection{Principles of Real-Time qPCR}

The basic principle of polymerase chain reaction (PCR) is the ability of DNA polymerase to synthesize a new strand of DNA complementary to an offered template strand [46]. Due to the nature of DNA polymerase, it can only add nucleotides to a preexisting 3' end and requires a primer to initially bind to a target strand of template DNA [46]. The necessity of a DNA primer allows for the selection of a specific sequence of nucleotides, or a selected gene, to be synthesized by DNA polymerase. To perform qPCR, the template DNA, the desired DNA primer, and a fluorescent marker are combined into a sample well and placed in a thermocycler equipped with a real-time qPCR system. For 
this study, SYBR green was used as the fluorescent marker because it is inexpensive and is not sequence-specific - allowing for use with any DNA primer. SYBR green is an intercalating dye that emits fluorescence when bound to double stranded DNA [39].

There are three steps to the reaction cycle as shown in Figure 7: (i) denaturation, (ii) annealing, and (iii) elongation. First, the samples are heated to $95^{\circ} \mathrm{C}$ for 20 seconds, causing strand separation of the template DNA by disrupting the hydrogen bonds between complementary bases, known as the denaturation step [46]. Next, the annealing step occurs when the samples are cooled to $60^{\circ} \mathrm{C}$ for 30 seconds, allowing the DNA primers to bond, or anneal, to the single-stranded DNA template. The DNA primers are composed of a specific sequence related to the gene of interest, and will only form DNADNA hydrogen bonds if the primer sequence closely matches the template sequence [46]. Finally, DNA polymerase is able to bind to the 3' end of the template DNA-DNA primer structure and synthesize a new complementary DNA strand from the template DNA strand. DNA polymerase adds nucleotides, also known as deoxynucleotide triphosphates (dNTPs), to the DNA primer in the elongation step. This concludes one cycle of PCR. During the elongation step, the fluorescent marker SYBR green binds to the formed double-stranded DNA and emits fluorescence. The amount of fluorescence is measured at the conclusion of each cycle. This process is repeated for 40 cycles, resulting in the exponential amplification of DNA. The real-time qPCR instrument plots the measured fluorescence against the cycle number, resulting in an amplification plot. The fluorescence on the amplification plot represents the accumulation of double-stranded DNA product over the duration of the entire PCR reaction. This is quantified as the $\mathrm{Ct}$, or cycle threshold, to reach a minimum amount of fluorescence. 


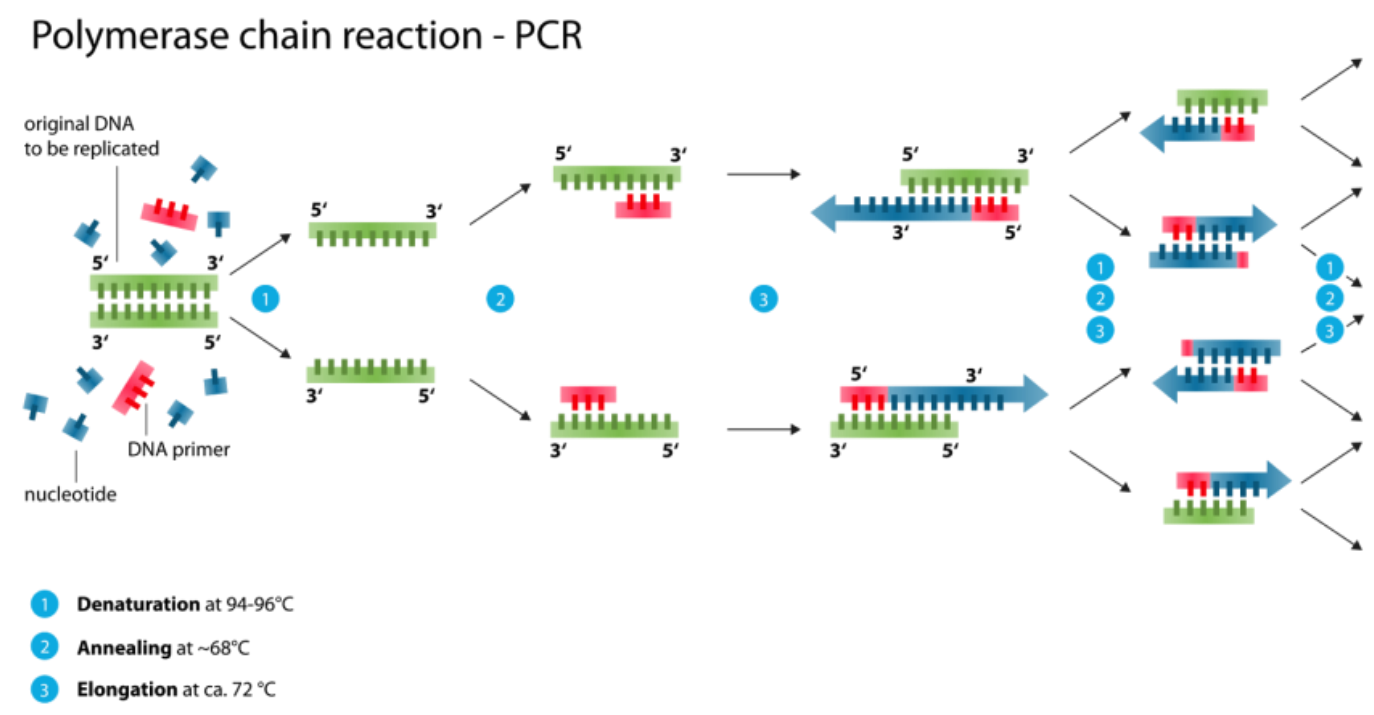

Figure 7: Operating principle of polymerase chain reaction [47].

\subsection{Methods}

\subsubsection{Isolating RNA}

In order to perform qPCR, messenger RNA (mRNA) had to be isolated from the tissue or cell samples. This was accomplished by lysing cells to access cell contents. Following the diagram in Figure 8, RNA purification was performed by adding a buffer solution and $70 \%$ ethanol to the lysate, and transferred to a spin column [48]. Spin columns use forces generated via centrifugation to filter lysate through a silica gel membrane coating [48]. The nucleic acid binds to the silica gel, while the other cell components continue to pass through. This process was repeated to remove membrane lipids, proteins, and other cellular contaminants and is referred to as "washing" steps. Finally, nuclease-free water was added to remove the nucleic acids from the membrane and the elution was collected for use in reverse transcription. The RNeasy Mini Kit (QIAGEN, Hilden, Germany) was used in this study, with the detailed protocol for RNA isolation provided in Appendix B. 


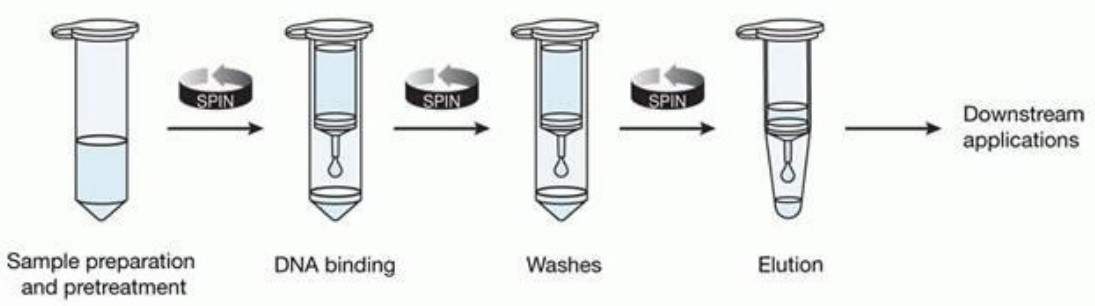

Figure 8: RNA purification using spin column methods. Source: www.thermofisher.com.

The isolated mRNA was then assessed for quantity and purity using concentration and ratio of absorbance at $260 \mathrm{~nm}$ and $280 \mathrm{~nm}(\mathrm{~A} 260 / 280)$ values obtained from a $1 \mu \mathrm{L}$ sample of isolated RNA and the NanoDrop 2000 UV-Vis Spectrophotometer (Thermo Fisher Scientific, Waltham, MA). The A260/280 value was used to assess the purity of RNA, where a ratio of 1.8 to 2.0 generally indicates good quality RNA that is devoid of protein contamination [49]. The concentration values of mRNA in each elution was used to calculate the available amount of RNA (ng) for cDNA reverse transcription. The minimum amount of RNA needed to perform cDNA synthesis is 200ng, which was the amount used for the preliminary study. A detailed protocol for RNA quality assessment is provided in appendix $\mathrm{C}$.

\subsection{2 cDNA Synthesis}

From the mRNA obtained from the biologic samples, reverse transcription was used to produce complementary DNA (cDNA). The volume of RNA isolate needed was calculated using equation 2.1. Reverse transcriptase and a short RNA-specific primeramong other reagents - are combined with the isolated mRNA to produce cDNA. This 
cDNA was then used as the template DNA in qPCR analysis. The detailed protocol for transcription is provided in Appendix D.

$$
(200 \text { ng RNA }) *\left(\frac{1 \mathrm{~mL}}{\left[\mathrm{RNA}^{\mathrm{ng}} / \mathrm{nL}\right.}\right)=\text { Volume RNA }(\mu \mathrm{L})(2.1)
$$

\subsubsection{Reference Gene Selection}

To complete the data analysis of the $\mathrm{Ct}$ values obtained from qPCR, a reference gene must be used as an internal reaction control for normalization and calculation of relative abundance of each gene of interest $[50,51]$. A reliable reference gene must meet several criteria: most importantly, it must be expressed at the same level under all experimental factors; it should show minimal variability in expression between cell types and physiological states of the tissue; and it should demonstrate variability resulting from imperfections of the technology and preparation methods $[50,51]$. The basic metabolism genes or HKGs, by definition, are involved in processes essential for the survival of cells, and therefore must be expressed in a stable and non-regulated constant level [52]. It is widely-held that the basic metabolism genes - also known as housekeeping genes (HKGs ) - fulfill these conditions best; however; this is not always true depending on the experiment. Many housekeeping genes are not only present in basic metabolic processes, but in other functions depending on the gene and tissue type [51]. For each tissue or cell type and specific experimental designs, a proper search and validation of reference gene is needed to ensure that there is no significant change in expression and to ensure accurate data analysis [53]. 
Expression of reference genes must be unaffected by differences in cell type or experimental treatment. In this experiment, HUVECs and DHCAECs were treated with elevated levels of glucose. A previous study has shown that glyceraldehyde-3-phosphate dehydrogenase $(G A P D H)$ and $\beta$-actin $(B A C T)$ both display stable expression in HUVECs cultured in a hyperglycemia model [54]. In the same study that found $R A G E$ expression increases in endothelial cells exposed to hyperglycemic conditions, GAPDH was used as a reference gene for their RT-qPCR analysis, with $B A C T$ used as an internal control in the event $G A P D H$ was not sufficient as a reference gene [42]. $G A P D H$ was also used as a reference gene in the study that found elevated $e N O S$ expression in HAECs when exposed to high glucose conditions [45]. Both BACT and GAPDH were included in the primer verification experiment to determine the optimal reference gene for the remainder of the study.

\subsubsection{Primer Design}

For each gene of interest, a different DNA primer is needed to run qPCR. Each gene of interest requires a set of two DNA primers: a forward and a reverse, for 5' to 3' synthesis on complementary strands of cDNA. There are validated primer sets for a myriad of species-specific genes, thus researchers may select their own primer sets for each gene relatively quickly using these available primer sets. In SYBR green-based qPCR, specificity of each primer is extremely important as SYBR green will fluoresce when bound to any double stranded DNA (dsDNA), regardless if the product is the desired gene, or amplicon [39]. Poor primer choice could result in increased fluorescence, therefore inaccurate quantification, from nonspecific binding of SYBR green to incorrect 
amplification products or primer dimer formation. Primer dimer formation occurs when the forward and reverse primers bind to each other rather than the template DNA. Proper primer selection leads to increased sensitivity of quantification and assay reproducibility. With SYBR green, the amplicon length is also important because the longer the product, the more dsDNA, which leads to increased fluorescence, which may affect comparison studies. The ideal primers for SYBR Green qPCR are highly specific, do not produce primer dimers, and have similar amplicon lengths ( $80-150 \mathrm{bp}$ ), which can be achieved through quality primer design [55]. One way to ensure specificity is to find primers 18-24 base pairs (bp) in length [55].

For this thesis, primer selection played an important role in the development of the qPCR protocol used. First, a gene sequence was identified for each gene of interest using the GenBank, the National Institutes of Health (NIH) genetic sequence database. Once a gene sequence was found that was specific to the gene of interest, mRNA, species, and base pair length, the sequence was cross-referenced using PrimerQuest provided by Integrated DNA Technologies. PrimerQuest output provided pre-validated DNA primers based on the gene sequence, type of qPCR, primer size (length), and amplicon size (length). Once a primer set is determined, the primer sequences were input in Primer-BLAST, an extension of the GenBank database provided by the NIH. This database provides the homology of primer sets. Homology is the existence of similar genes that share a primer. For optimal primers, high homology with different variants of the same gene and low homology with non-target genes is desired. Appendix G provides a detailed protocol for primer design. Two or three primer sets were selected for each 
gene of interest and the reference genes. A table of all primer sets included in the verification is shown in appendix $H$.

\subsubsection{Primer Verification}

To verify the efficiency of the selected primers, a qPCR plate was run with a sample of cDNA isolated from HUVECs grown in static culture in normal media. Technical triplicates were run for each primer set, as well as a non-template control (NTC) as shown in the 96-well plate template in Figure 9. Non-template controls do not contain any cDNA and can be used to check for primer contamination and primer dimer formation.

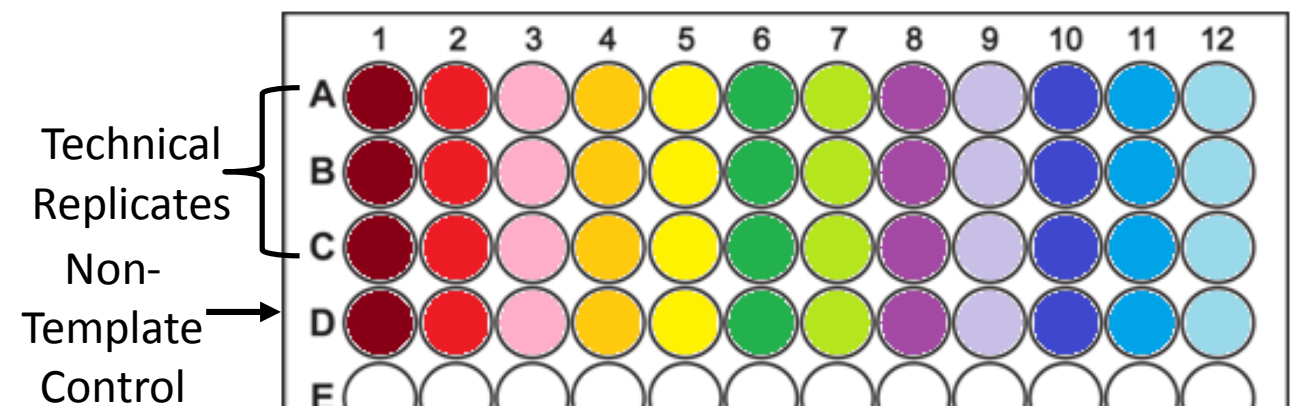

Figure 9: 96-well plate template, with each color representing different primer, for primer verification.

\subsection{6 qPCR Data Analysis}

The raw data obtained from qPCR is the crossing threshold, $\mathrm{Ct}$, or the number of cycles that it takes the reaction to reach a minimum amount of fluorescence. This requires some calculation and interpretation to ascertain the quantification of each gene. This analysis 
can determine absolute or relative levels. Absolute quantification involves the calculation of number of copies of a specific RNA per sample for a given gene of interest (GOI) [39]. Relative quantification determines the level of gene expression for one sample compared to another sample, which is more widely used and is used for this thesis. The $2^{-\Delta \Delta \mathrm{Ct}}$ method is the most commonly used to determine relative quantitation [39].

The $2^{-\Delta \Delta C t}$ method operates under the assumption that PCR efficiency is $100 \%$, such that the PCR product doubles after every cycle [39]. This method also requires the use of a reference gene that have a stable expression level in all tissues or cells undergoing investigation [54]. Ideally, this reference gene is not regulated or affected by experimental conditions. In this analysis, the reference gene is used to normalize the data for each sample by determining the $\Delta \mathrm{Ct}$ value as shown in equation 2.2.

$$
\Delta C t=C t_{\text {sample } G O I}-C t_{\text {sample, reference }}
$$

From the $\Delta \mathrm{Ct}$ value, the $\Delta \Delta \mathrm{Ct}$ value and relative abundance are calculated using equations 2.3 and 2.4 , respectively.

$$
\begin{aligned}
& \Delta \Delta C t=\Delta C t_{\text {experimental }}-\Delta C t_{\text {control }} \\
& \text { Abundance }=2^{-\Delta C t}
\end{aligned}
$$

Then the $\Delta \Delta \mathrm{Ct}$ value is used to determine the fold change using equation 2.5.

$$
\text { Fold Change }=2^{-\Delta \Delta C t}
$$

The fold change value represents the level of the gene of interest in the experimental sample as a percentage of the level of the gene of interest in the control sample [39]. For instance, a value of 1.5 indicates the gene of interest is expressed $150 \%$ more in the experimental sample compared to the control sample. This method effectively produces a 
linear relationship for standard statistical tests, such as Student's t-test or analysis of variance (ANOVA), from the exponential Ct values [39].

In this thesis, the abundance calculation was used to determine the level of gene expression because the loss of the control data point in the fold change calculation is undesirable. By using abundance, the $\mathrm{Ct}$ values are still transformed into data with a linear relationship for comparative statistics. Abundance can be interpreted as the percentage of expression for the gene of interest relative to the reference gene. A detailed protocol of qPCR data analysis is provided in appendix F.

\subsection{Results: qPCR Primer Verification}

In order to choose the best primer for each gene, the amplification and melting curves can be used to determine the quality of each primer set. An amplification plot is the derivative of measured fluorescence as a function of cycle number, while the melting - or dissociation - curve is the derivative of measured fluorescence as a function of temperature as the thermocycler heats through the dissociation temperature of the amplicon [56]. Fluorescence signals occur as a result of SYBR green binding to dsDNA, therefore fluorescence is directly proportional to the amount of dsDNA in each sample.

The amplification curve is used to determine the efficiency of PCR, as well as the threshold for which $\mathrm{Ct}$ values are obtained for each sample [39]. For primer verification, the only variable between each well was the primer set therefore the information obtained from the amplification curve is most likely due to primer efficiency. An ideal amplification curve shows the reaction going through exponential and linear phases until PCR reagents become exhausted, at which point the reaction reaches a plateau, as seen in 
Figure 10. Assuming there are exactly equal amounts of cDNA and other reagents in each well, it would also be expected that exponential amplification of each sample would occur around the same cycle number, or have similar $\mathrm{Ct}$ values. The NTC was used to verify primer dimer formation, which would be visualized as amplification in the NTC well. From the first primer set chosen for $G A P D H$, there was amplification of the NTC at curve 1 in Figure 10A. From the third primer set chosen for $G A P D H$, the amplification curve revealed primer efficiency with similar $\mathrm{Ct}$ values for both samples, seen at the curves crossing the threshold at approximately the same cycle in Figure 10B at curve 2. There was also no amplification of the NTC, seen by the lack of a curve at 3 in Figure $10 B$. 


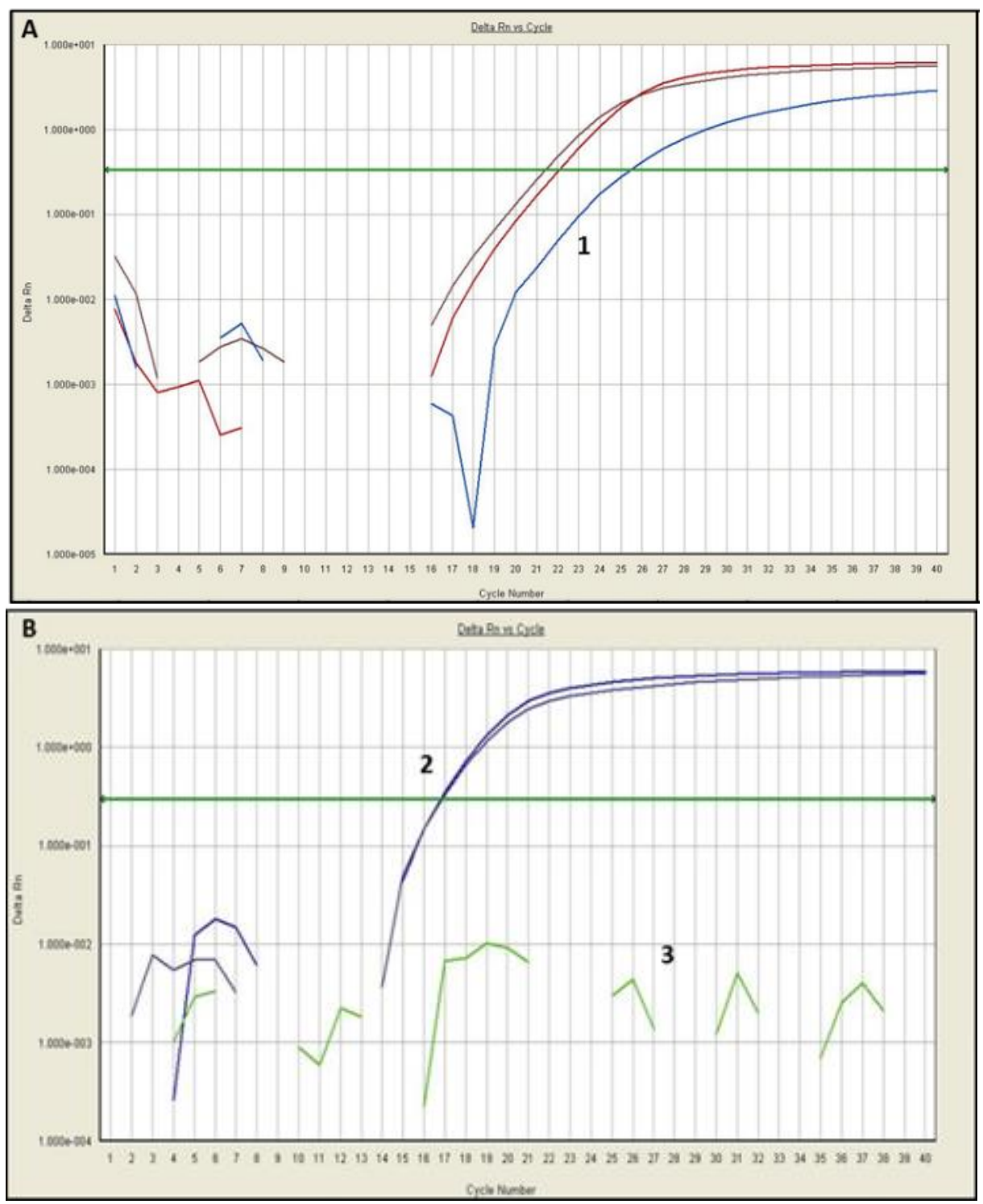

Figure 10: Amplification curves from primer verification for two different primer sets for GAPDH.

Since SYBR green non-specifically binds to dsDNA, primer dimer formation and other artifacts can lead to error in the amplification curves. To ensure that the desired product was amplified, a dissociation cycle is performed after amplification cycles are completed [39]. The dissociation cycle increases the temperature incrementally while fluorescence is 
measured. As temperature increases, DNA denatures into single strands and fluorescence decreases. Dissociation temperature correlates with the length of the amplification product, as dissociation temperature increases with product length. Primer dimer formation can be identified by significantly lower dissociation temperatures - due to their shorter length (18-22bp) - than the desired products (>200bp). In studies of qPCR accuracy, it has been found that the analysis of melting curves can distinguish desired products from undesired products, eliminating the need for agarose gel electrophoresis of qPCR products [56].

The dissociation curves in Figure 11 correspond with the primer sets used for Figure 10. Figure 11A shows primer dimer formation by dissociation curve 1 . The amplicon of this primer set was also non-ideal as the dissociation curves for two samples did dissociate at the same temperature, seen by the curves at 2. Figure 11B shows that the NTC did not have any products or primer dimer formation, seen by the flat line at 3. The amplicons in the sample wells have close dissociation temperatures as seen at 4.

The amplification curve also showed that all sample wells had reasonable $\mathrm{Ct}$ values for the genes of interest. More specifically, the $\mathrm{Ct}$ values for the same primerwhich is specific to each gene of interest - were relatively similar, indicating appropriate amplification efficiency. 


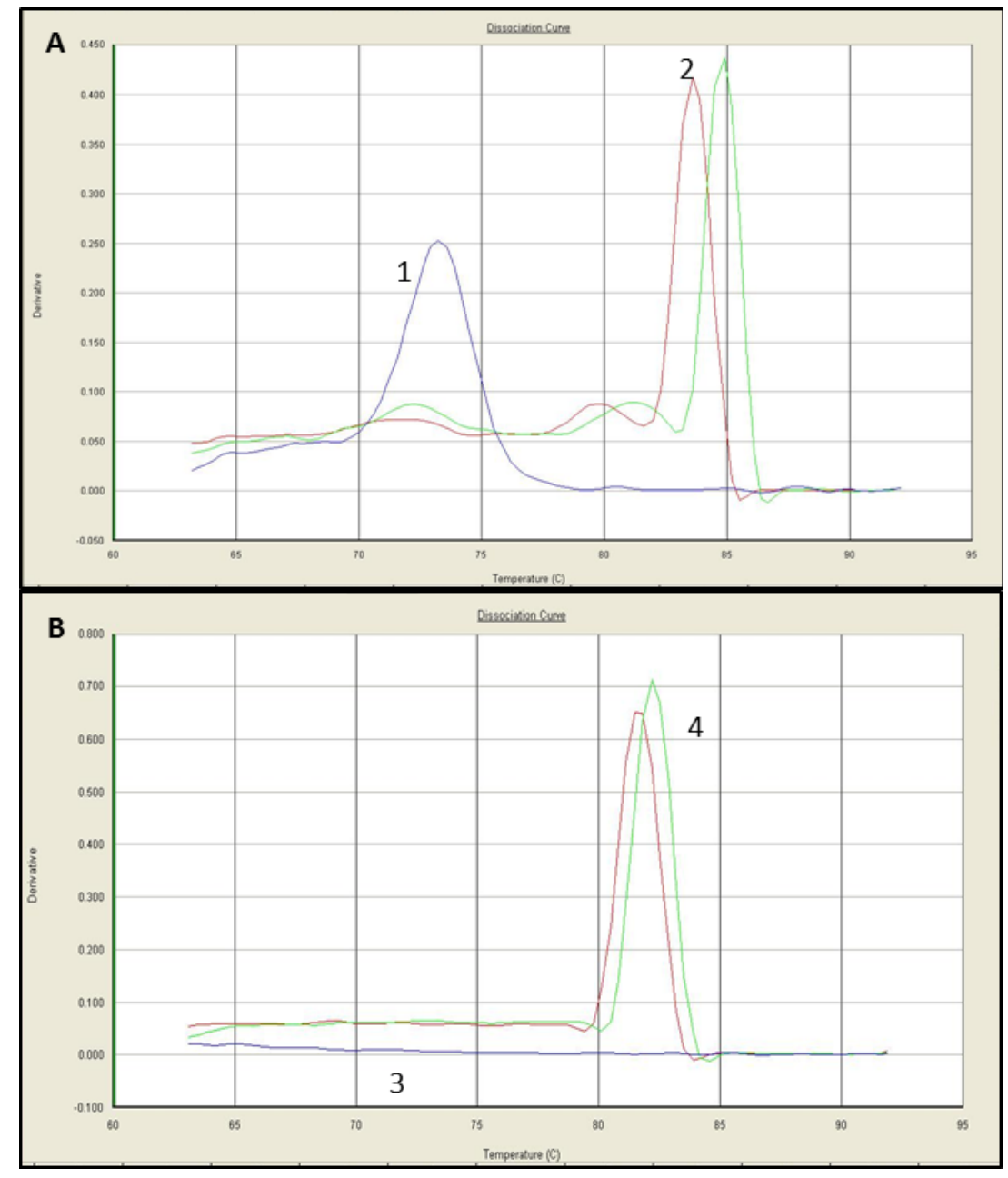

Figure 11: Dissociation curves from primer verification for two different primer sets of GAPDH.

From the analysis of the amplification and dissociation curve for each primer set, a single primer set for each gene of interest and reference gene was selected for use in the remainder of this study. The dissociation curves were scrutinized especially for primer dimer formation as the primer denaturation temperature was provided by the manufacturer for each primer. The selected primer sets and sequences for each gene are given in Table 2. 
Table 2: Primer sets and sequences for each gene of interest and reference genes.

\begin{tabular}{llccl}
\hline \multicolumn{1}{c}{ Primer ID } & Direction & Sequence (5' to 3') & $\begin{array}{c}\text { Length } \\
\text { (bp) }\end{array}$ & Homologs \\
\hline GAPDH & Forward & GTCGGAGTCAACGGATTTG & 19 & Self: 3 \\
$($ NM_001289745.1) & Reverse & TCCATTGATGACAAGCTTCC & 20 & Other: 0 \\
PECAM & Forward & CCAGCCCAGGATTTCTTATG & 20 & Self: 7 \\
$($ NM_000442.4) & Reverse & TCTGCAACACACTGGTATTC & 20 & Other: 0 \\
$R A G E$ & Forward & AGCGTGCAGAACTGAATC & 18 & Self: 8 \\
(NM_001136.4) & Reverse & GGCTCTGGTTGTAGAAGAAA & 20 & Other: 1 \\
eNOS & Forward & GTCCTGTGTATGGATGAGTATG & 22 & Self: 2 \\
(NM_000603.4) & Reverse & GCTGTTGAAGCGGATCTT & 18 & Other: 0 \\
\hline BACT was excluded after $G A P D H$ & was selected as the reference gene from the pilot & study.
\end{tabular}

\subsection{Conclusion}

This preliminary study was used to show that qPCR analysis of the selected genes of interest and reference gene is feasible. The qPCR data showed reasonable amplification and dissociation curves, with minimal primer-dimer formation for each primer selected. The verification study also showed the formation of a single PCR product, or amplicon, for each gene of interest. It was also found that GAPDH was the optimal reference gene over BACT. The qPCR data indicated successful completion of the first aim of this thesis, to establish a qPCR protocol for the analysis of HUVECs and DHCAECs. 


\section{Chapter 3: Preliminary Study to Develop a Hyperglycemic Condition In Vitro}

\subsection{Introduction}

In order to investigate the effect of a simulated hyperglycemic environment on HUVECs and DHCAECs in tissue engineered BVMS, a protocol had to be developed for replicating a hyperglycemic condition in vitro. A preliminary study was conducted to determine the optimal concentration of glucose to add to cell culture to elicit cellular responses, without causing apoptosis. For time and cost efficiency, this initial study was conducted using HUVECs and DHCAECs grown in flasks and not in a BVM construct. The primary outcome of this study was to determine a single glucose concentration to use for the creation of "diabetic" BVMs, and to demonstrate the feasibility of creating a more accurate diabetic blood vessel model.

Numerous studies have shown that HUVECs subjected to high glucose concentrations in culture may undergo increased apoptosis, or other alterations in cellular processes, through various mechanisms associated with diabetes [54, 55, 57]. Previous studies used the same control - a normal glucose concentration of $5 \mathrm{mM}$ - in cell media; however, all studies used different concentrations to represent their high glucose condition. These studies also differed in the duration of exposure to the high glucose culture conditions, as shown in table 3. From this information, it was determined that two levels of high glucose concentrations would be used in the preliminary study to determine the extent of apoptosis in HUVECs and DHCAECs. Cell culture media used for the culture of endothelial cells typically contain $5.5 \mathrm{mM}$ of glucose, which approximates normal blood sugar levels in vivo $[31,58,59]$. The second glucose concentration 
investigated was $10.5 \mathrm{mM}$ of glucose as a moderate level, that reflects pre-diabetic blood glucose levels, and represents a glycemic threshold when diabetic complications arise in vivo [31]. From the studies given and expected time points for a BVM set up in future studies, a concentration of $25.5 \mathrm{mM}$ of glucose was selected as the high glucose level.

Table 3: Glucose conditions, time, study [37, 42, 43, 45].

\begin{tabular}{lccc}
\hline Study & $\begin{array}{c}\text { High Glucose } \\
\text { Concentration }\end{array}$ & $\begin{array}{c}\text { Duration of } \\
\text { Exposure }\end{array}$ & Outcome \\
\hline $\begin{array}{l}\text { Bakhashab S, et al. }- \\
\text { Reference Genes... }\end{array}$ & $16.5 \mathrm{mM}$ & 1 day & n/a \\
$\begin{array}{l}\text { Cosentino F, et al. }- \text { High } \\
\text { glucose increases nitric oxide }\end{array}$ & $22.2 \mathrm{mM}$ & 5 days & $\begin{array}{l}\text { Increased } \text { eNOS gene } \\
\text { expression }\end{array}$ \\
$\begin{array}{l}\text { synthase... } \\
\begin{array}{l}\text { Piconi L, et al. - Constant and } \\
\text { intermittent high glucose... }\end{array}\end{array}$ & $20 \mathrm{mM}$ & Up to 14 days & $\begin{array}{l}\text { Increased apoptosis } \\
\text { due to ROS } \\
\text { overproduction } \\
\text { Cell survival } \leq 24 \mathrm{~h} \\
\text { Apoptosis } ~ 48 \text { hours }\end{array}$ \\
$\begin{array}{l}\text { Ho FM, et al. - High-glucose } \\
\text { induced apoptosis... }\end{array}$ & $33 \mathrm{mM}$ & Up to 2 days & \begin{tabular}{l} 
Aposis \\
\hline
\end{tabular}
\end{tabular}

\subsection{Materials and Methods}

Human umbilical vein endothelial cells (HUVECs) and type II diabetic human coronary artery endothelial cells (DHCAECs) (Lonza, Basel, Switzerland) were cultured under standard conditions $\left(37^{\circ} \mathrm{C}, 5 \% \mathrm{CO}_{2}\right)$ in endothelial growth media (Lonza EGM-2, cc3162) and coronary endothelial cell media (Lonza EGM-2 MV, cc3202), respectively. Endothelial cells were trypsinized and passed from a T75 cell culture flask at approximately $90 \%$ confluency at a ratio of 1:3 to allow for growth. Cell media was replaced every 2 days. This process was repeated until four T75 flasks were obtained for each cell type. After 24 hours -to allow for adhesion of cells to the flask - normal cell 
media was replaced with the experimental treatments as shown in table 4 . The remaining flasks of cells were frozen down for future use.

Table 4: Treatment groups to determine glucose concentration.

\begin{tabular}{|c|c|c|}
\hline & \multicolumn{2}{|c|}{ Cell Type } \\
\hline \multirow{4}{*}{ 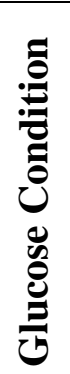 } & HUVECs + Control Media & DHCAECs + Control Media \\
\hline & HUVECs + Moderate & DHCAECs + Moderate \\
\hline & Glucose & Glucose \\
\hline & HUVECs + High Glucose & DHCAECs + High Glucose \\
\hline
\end{tabular}

\subsubsection{Media Preparation}

For this preliminary study, a 0.08M glucose (Sigma Aldrich, St. Louis, MO) solution was prepared and autoclaved. Using equations 2.2 and 2.3, the appropriate amount of glucose solution, shown in table 5, was added to cell media to obtain the desired treatment groups.

$$
\begin{gathered}
C_{\text {glucose }} V_{\text {glucose }}=C_{\text {desired }}\left(V_{\text {media }}+V_{\text {glucose }}\right) \\
\frac{\left(C_{\text {glucose }} V_{\text {glucose }}+C_{\text {media }} V_{\text {media }}\right)}{V_{\text {glucose }}+V_{\text {media }}}=C_{\text {desired }}
\end{gathered}
$$

Table 5: Media preparation specifications.

\begin{tabular}{cccc}
\hline $\begin{array}{l}\text { Final Glucose } \\
\text { Concentration of } \\
\text { Media }\left(\mathbf{m m o l} \cdot \mathbf{L}^{-\mathbf{1}}\right)\end{array}$ & $\begin{array}{l}\text { Volume } \\
\text { Glucose } \\
\text { Solution } \\
(\mathbf{m L})\end{array}$ & $\begin{array}{l}\text { Volume of } \\
\text { Cell Media } \\
(\mathbf{m L})\end{array}$ & $\begin{array}{l}\text { Total } \\
\text { Volume } \\
(\mathbf{m L})\end{array}$ \\
\hline 10.5 & 18.0 & 250 & 268.0 \\
25.5 & 91.7 & 250 & 341.7 \\
\hline
\end{tabular}




\subsubsection{Immunofluorescence}

After 7 days of cultivation in the specified glucose environment, cells from each flask were trypsinized to remove them from each flask, transferred to a $50 \mathrm{~mL}$ conical, and centrifuged to form a cell pellet. The pellet was resuspended in the appropriate glucose media and seeded at approximately $1.2 \times 10^{6}$ cells $/ 3 \mathrm{~mL}$ per well, with duplicate wells for each treatment according the Figure 12.
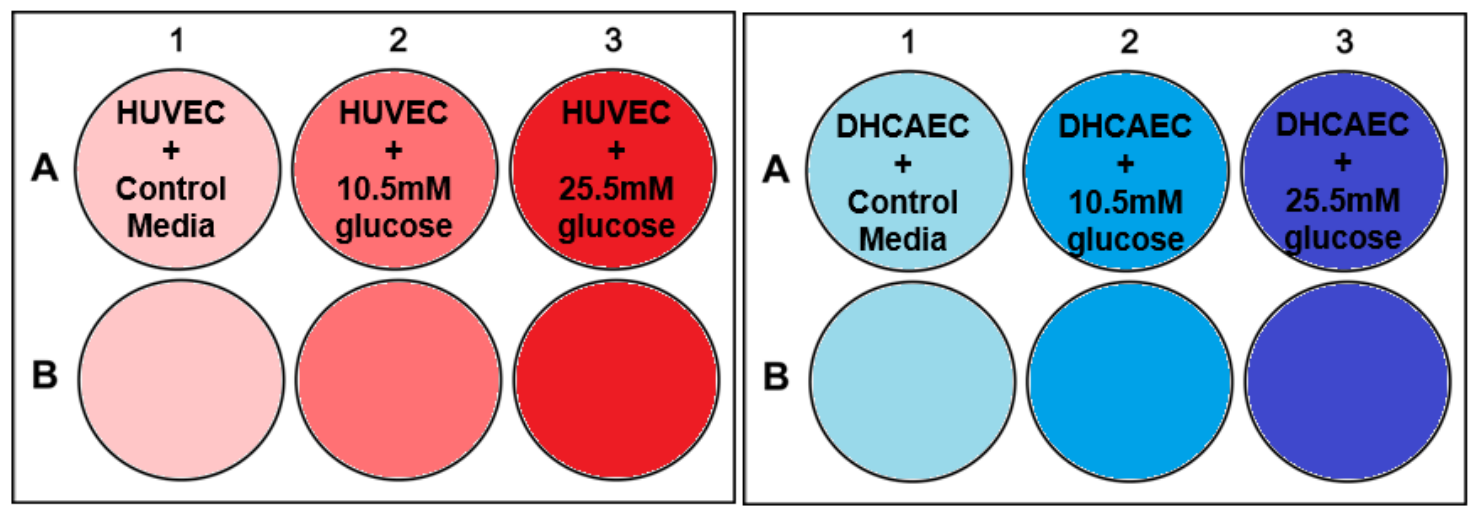

Figure 12: Sample and treatment scheme for immunofluorescence analysis.

Cells were cultivated in the wells for 24 hours, then fixed using $10 \%$ formalin buffered fixative (Azer Scientific, Morgantown, PA) applied in wells. Each well was gently washed with $1 \mathrm{~mL}$ of phosphate-buffered saline (PBS) (Thermo Fisher Scientific, Waltham, MA) to remove any remaining media or fixative. Then a $1 \% \mathrm{w} / \mathrm{w}$ solution of saponin in PBS was applied to each well to permeabilize the cell membranes. The saponin solution was aspirated and the wells are washed again in PBS. To prevent nonspecific binding of antibodies, the samples were blocked using fetal bovine serum (FBS) prior to the application of the PECAM primary antibody. The samples with the primary antibody were incubated 8-10 hours before being washed in PBS and the application of 
the secondary antibody, $\alpha$-mouse anti-CD31 (PECAM). The secondary antibody was incubated at room temperature for 60 minutes before another washing. Finally, a 1:400 solution of bisbenzimide (BBI) in PBS was applied for 5 minutes and a final wash of PBS was completed. All liquids were removed, and each sample was imaged using the specifications in table 6 at 10X objective magnification on the widefield fluorescent microscope.

Table 6: Settings used to obtain immunofluorescence images.

\begin{tabular}{ccc}
\hline Stain & $\begin{array}{l}\text { Excitation } \\
\text { Filter }(\mathbf{n m})\end{array}$ & $\begin{array}{l}\text { Emission } \\
\text { Filter }(\mathbf{n m})\end{array}$ \\
\hline BBI & $350 / 50$ & $457 / 50$ \\
PECAM & $490 / 20$ & $528 / 38$ \\
\hline
\end{tabular}

\subsubsection{Gene Expression}

From the same samples used for immunofluorescence, the remaining cells suspended in media were centrifuged into a cell pellet, the remaining media was aspirated, and the pellet was frozen in liquid nitrogen for qPCR analysis. Total RNA was extracted from each sample using the RNeasy Mini Kit (QIAGEN, Hilden, Germany) according to the manufacturer's instructions. The isolated RNA was assessed for quality and quantity using a NanoDrop 2000 UV-Vis Spectrophotometer (Thermo Fisher Scientific, Waltham, MA) to obtain concentration, and A260/280 values for each sample. Spectrophotometer readings were repeated three times for each sample of isolated RNA to minimize error that may arise from non-homogeneity of RNA concentration in the isolated elution. From the three readings, average RNA concentration values were determined and used to calculate volume needed for reverse transcription of RNA. For reverse transcription of 
RNA, $200 \mathrm{ng}$ of RNA is required for each sample therefore the volume of isolated RNA needed of each sample was calculated using the average RNA concentration and equation 2.1. Concentration, A260/280 and RNA volume values for each sample are provided in appendix I.

$$
\text { (200ng } R N A) *\left(\frac{1 m L}{[R N A]^{n g} / n L}\right)=\text { Volume } R N A(\mu L)
$$

Complementary DNA was synthesized from the RNA samples using the iScript cDNA Synthesis Kit (Bio-Rad, Hercules, CA) according to manufacturer's instructions to obtain a total volume of $20 \mathrm{uL}$ of cDNA. This cDNA solution was diluted 1:5 in nuclease-free water to obtain a total of $100 \mathrm{uL}$ of cDNA solution.

DNA primers (Integrated DNA Technologies, Coralville, IA) were diluted with nuclease-free water from stock solutions of $100 \mu \mathrm{M}$ to working solutions of $15 \mu \mathrm{M}$. A MicroAmp® fast optical 96-well reaction plate (Applied Biosystems, Foster City, CA) was prepared with technical triplicates for each treatment group and a non-template control (NTC), as shown in Figure 13, and full plate schemes given in table J. Each well contained $3.2 \mu \mathrm{L}$ of cDNA, or nuclease-free water for NTCs, $5 \mu \mathrm{L}$ Fast SYBR Green Master Mix (Life Technologies, Carlsbad, CA), $2 \mu \mathrm{L}$ forward primer, $2 \mu \mathrm{L}$ reverse primer, and $7.8 \mu \mathrm{L}$ of nuclease-free water for a total well volume of $20.0 \mu \mathrm{L}$. 


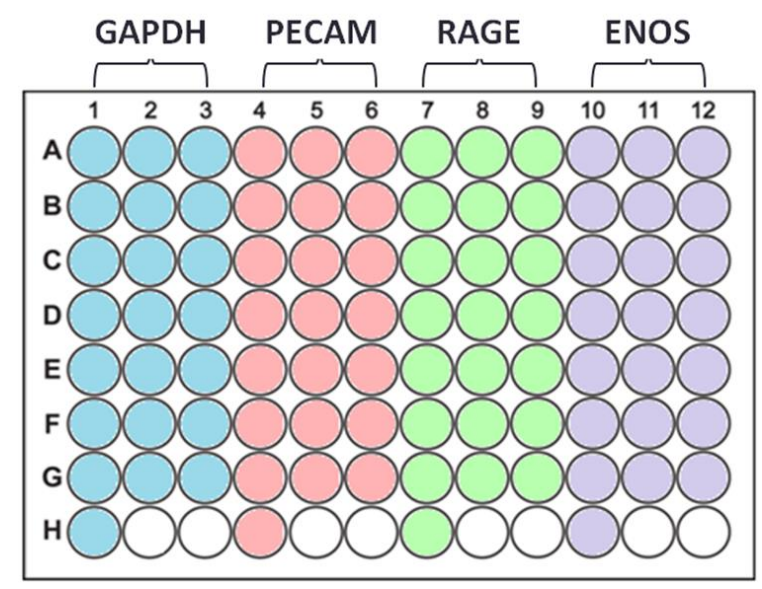

Figure 13: Example of 96-well template used to load samples, where each row represents a different sample group apart from row H: nontemplate controls.

Real-time qPCR was performed using 7500 Real-Time PCR Systems (Applied Biosystems, Foster City, CA) under the conditions given in table 7 for thermal cycling. As previously explained, during thermal cycling the polymerase chain reaction occurs: denaturation, annealing, and elongation of DNA - resulting in amplification of the desired genes and a measurement of fluorescence after each cycle.

Table 7: Thermocycling settings for 7500 Real-Time PCR System, cycled 40 times.

\begin{tabular}{cc}
\hline Temperature & Duration \\
\hline 95.0 & 20 secs \\
95.0 & 3 secs \\
60.0 & 30 secs \\
\hline
\end{tabular}




\subsubsection{Statistical Analysis}

Mean expression values of each technical triplicate were calculated using the $2^{-\Delta \mathrm{Ct}}$ method described in chapter 1, using GAPDH as the reference gene in equations 1.1 and 1.2 to obtain mean relative abundance. Abundance is presented as mean \pm SEM. The comparison of gene expression levels between treatment groups was performed using two-sample t-tests with Minitab Statistical Software.

\subsection{Results}

\subsubsection{Observations of Culture}

During the expansion of HUVECs and DHCAECs, it was found that the DHCAECs were consistently less confluent than the HUVECs over the same time period. Once the glucose treatments were implemented, it was also noted that as glucose concentration increased confluency decreased across both cell types. As the normal media groups appeared approximately $95-100 \%$ confluent at the time point investigated, the $10.5 \mathrm{mM}$ glucose media groups appeared $85-90 \%$ confluent, and the $25.5 \mathrm{mM}$ glucose media groups appeared approximately 80-90\% confluent.

\subsubsection{Immunofluorescence}

A representative image for each stain is presented for each treatment group with a third composite image that shows the images for each stain overlaid using ImageJ Software. Comparing HUVEC immunofluorescence images in Figure 14 and DHCAEC images in Figure 15, HUVECs appeared to have higher confluency given the number of distinguishable nuclei in the BBI images. For the DHCAEC images, it appears that the 
confluency decreased from the control media treatment (Figure 15A) to the $10.5 \mathrm{mM}$ glucose media treatment (Figure 15D) and from the control media treatment to the 25.5mM glucose media treatment (Figure 15G). It is difficult to discern differences in PECAM presence, as it is relative to the number of cells present in the given image; however, all samples stained positive for PECAM.

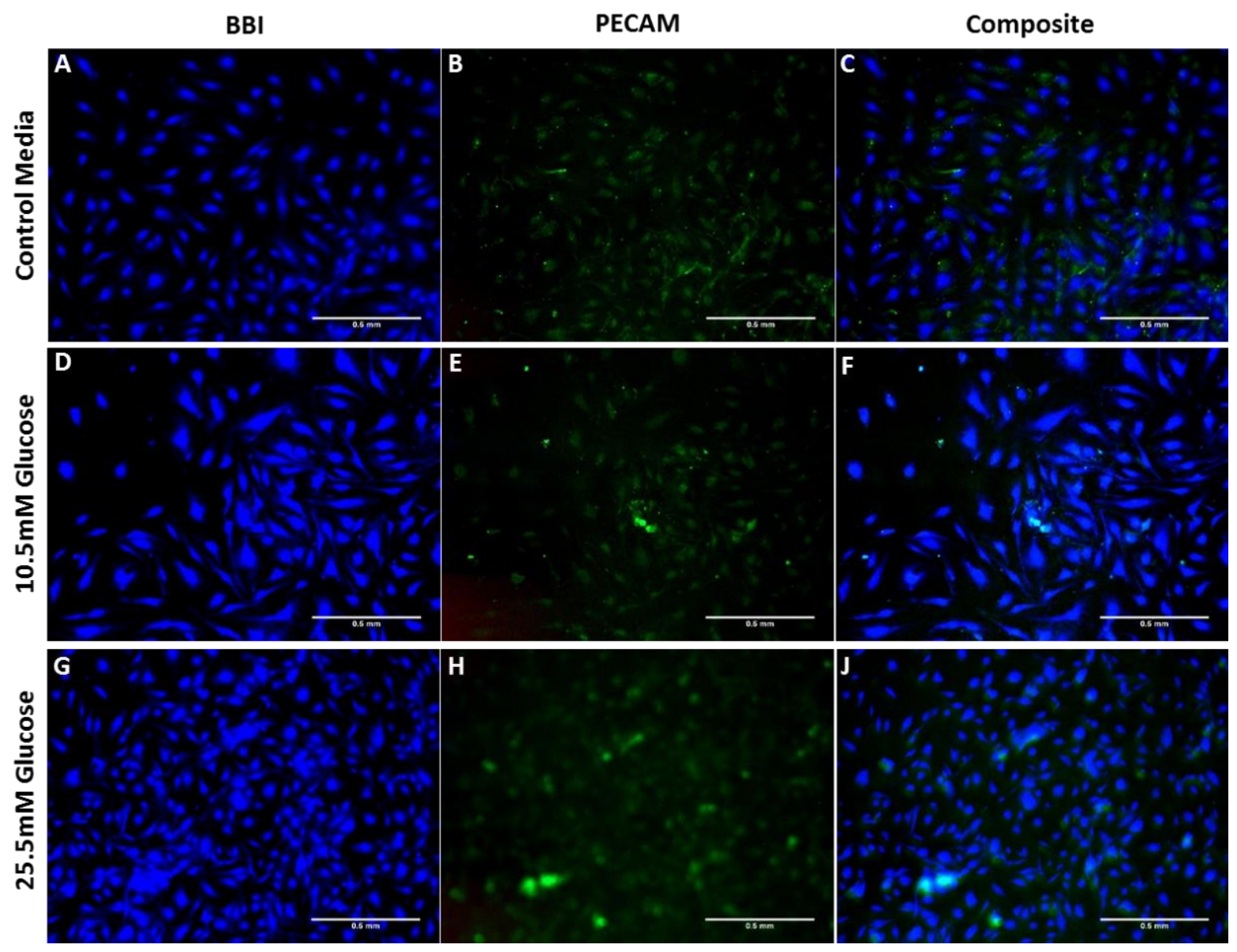

Figure 14: Immunofluorescence staining of HUVECs at 10x objective magnification. 


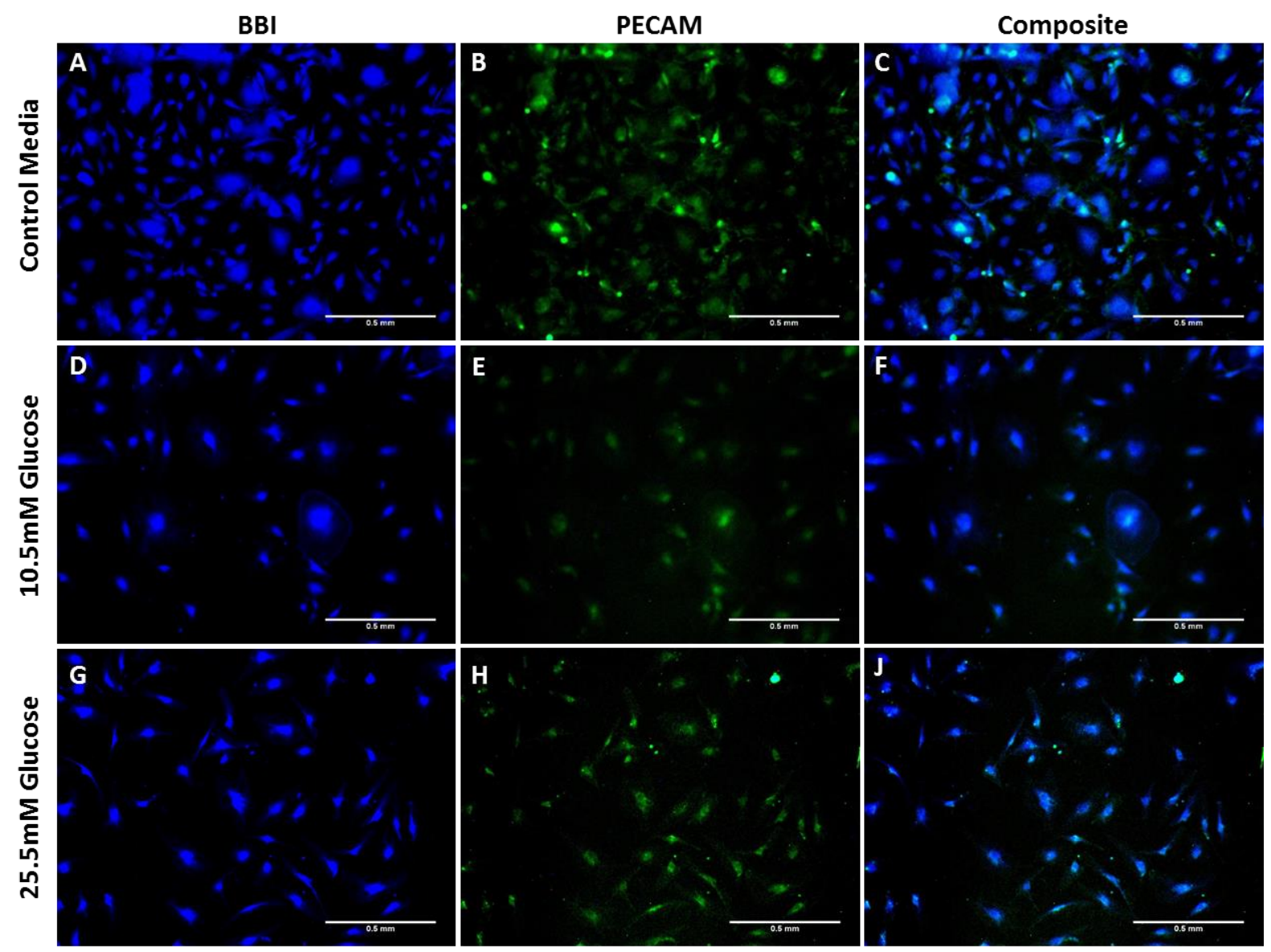

Figure 15: Immunofluorescence staining of DHCAECs at 10x objective magnification.

\subsubsection{Gene Expression}

The data obtained from qPCR was first inspected using amplification and dissociation curves for each gene as quality control. This was to ensure that the assumption of $100 \%$ PCR efficiency required to perform $2^{-\Delta C t}$ analysis, as mentioned in chapter 1 , is met for each sample. The dissociation curve was used to determine the presence of primer dimer formation, contamination, or multiple qPCR products. If a well did not meet quality control standards, the data point was removed from analysis. 
GAPDH - From the amplification and dissociation curves shown in Figures 16 and 17, respectively, it was found that all wells met quality control standards. The dissociation curve showed one sample that appeared to have some primer dimer formation, by the curve that peaked at a lower temperature comparatively; however, this well was identified as the NTC. There was no other primer dimer formation in the experimental samples, as seen by the lack of fluorescence signaling in any other sample. This primer dimer formation in the NTC also explains the amplification curve that appeared much later than the rest of the samples. The amplification curve showed acceptable efficiency and the dissociation shows a single amplicon in all sample wells.

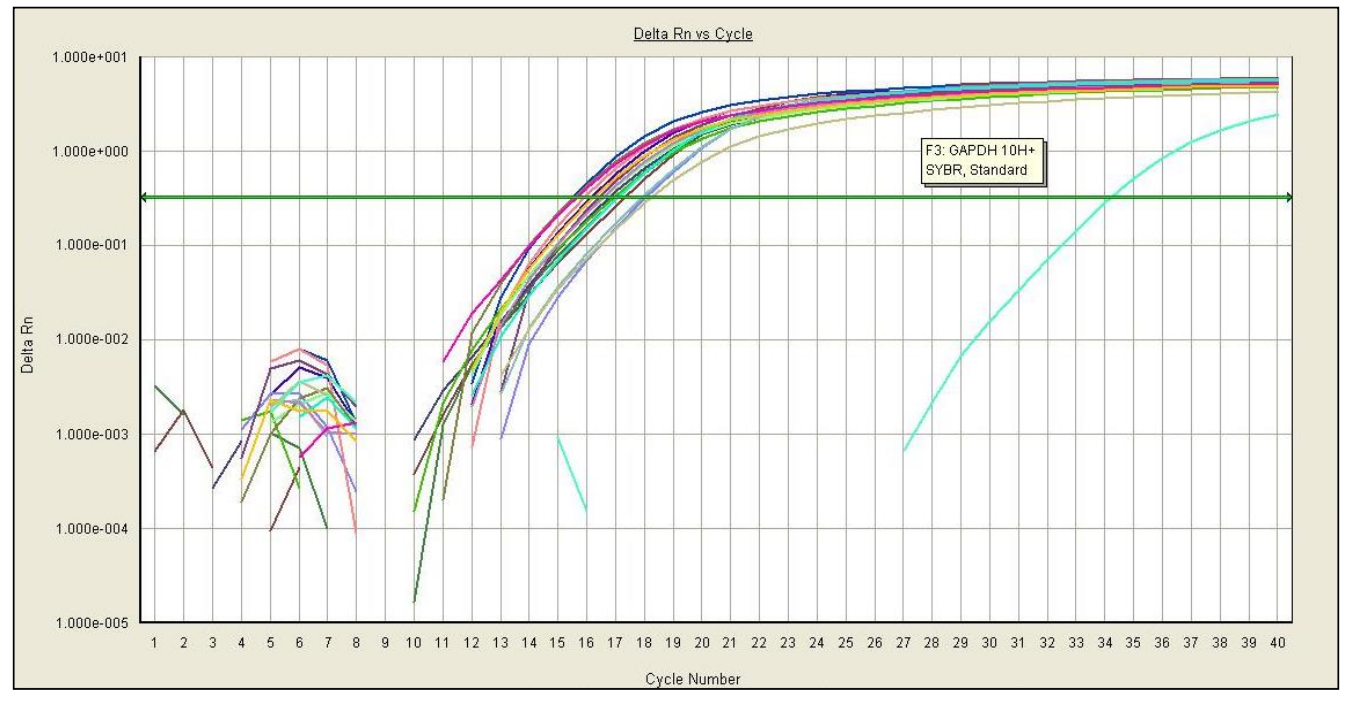

Figure 16: Amplification curve for GAPDH from 7500 Real-Time qPCR System. 


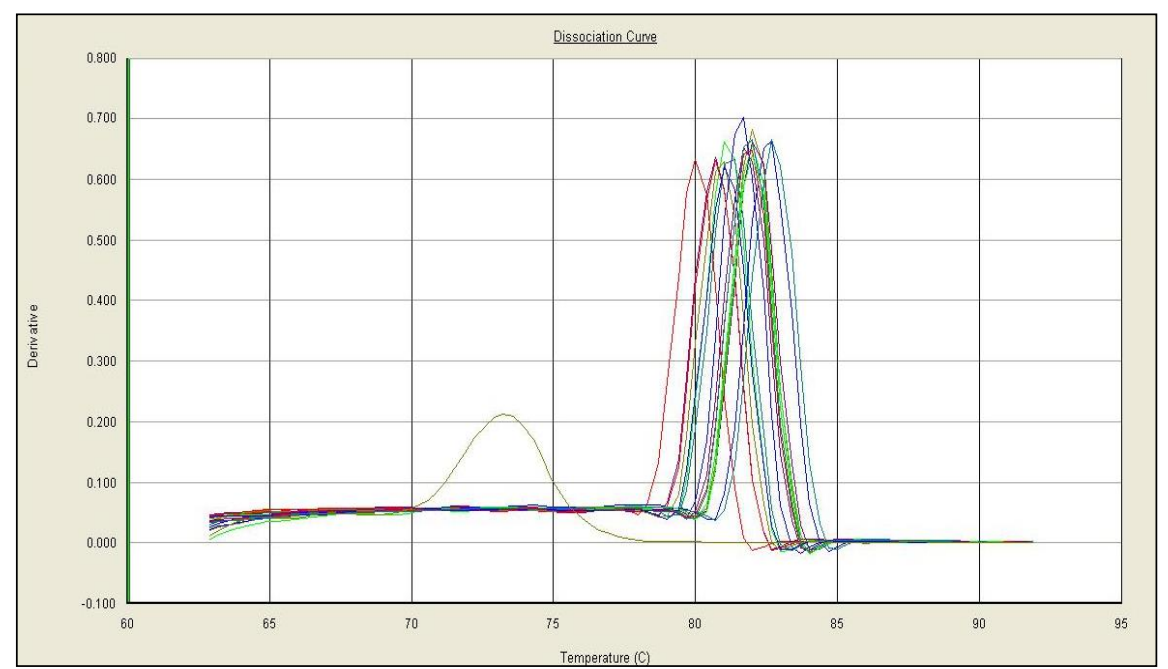

Figure 17: Dissociation curve for GAPDH from 7500 Real-Time qPCR System.

PECAM - The amplification curve for PECAM in Figure 18 showed acceptable PCR efficiency in all wells, with the appropriate absence of amplification in the NTC well. The dissociation curve in Figure 19 revealed a non-desired product was amplified in a well corresponding to a sample from HUVECs grown in $10.5 \mathrm{mM}$ of glucose, and was removed from analysis. In all other wells, the dissociation curve showed one product, no primer dimer formation, and an appropriate lack of product in the NTC.

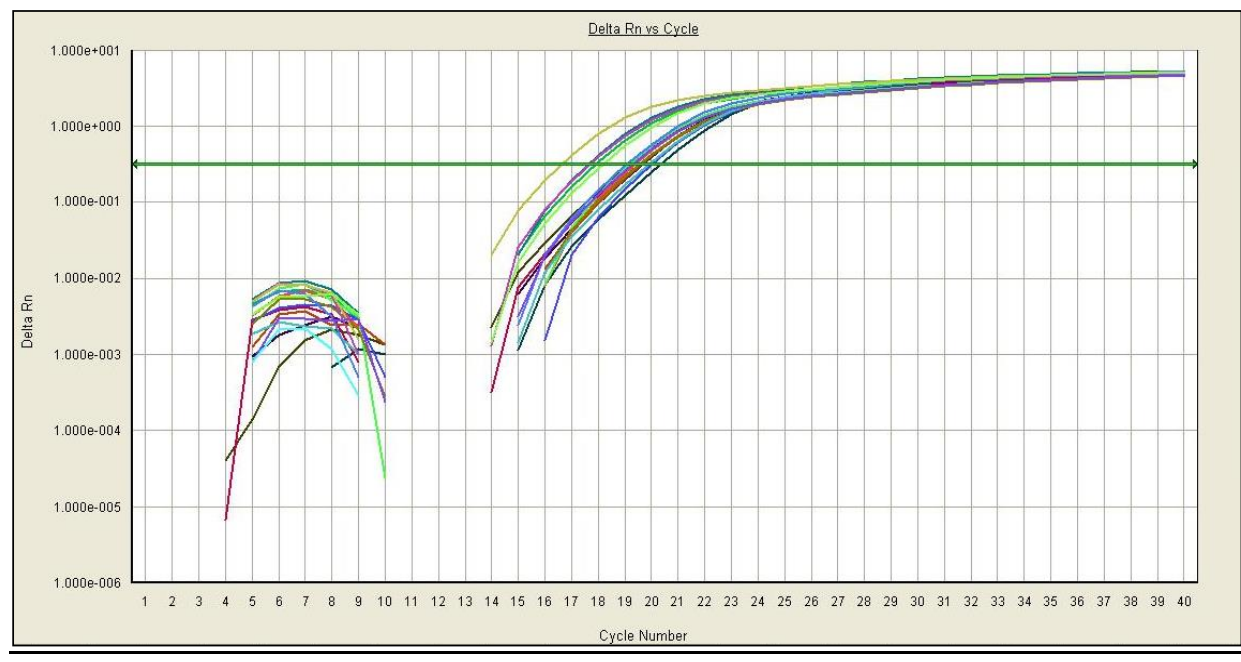

Figure 18: Amplification curve for PECAM from 7500 Real-Time qPCR System. 


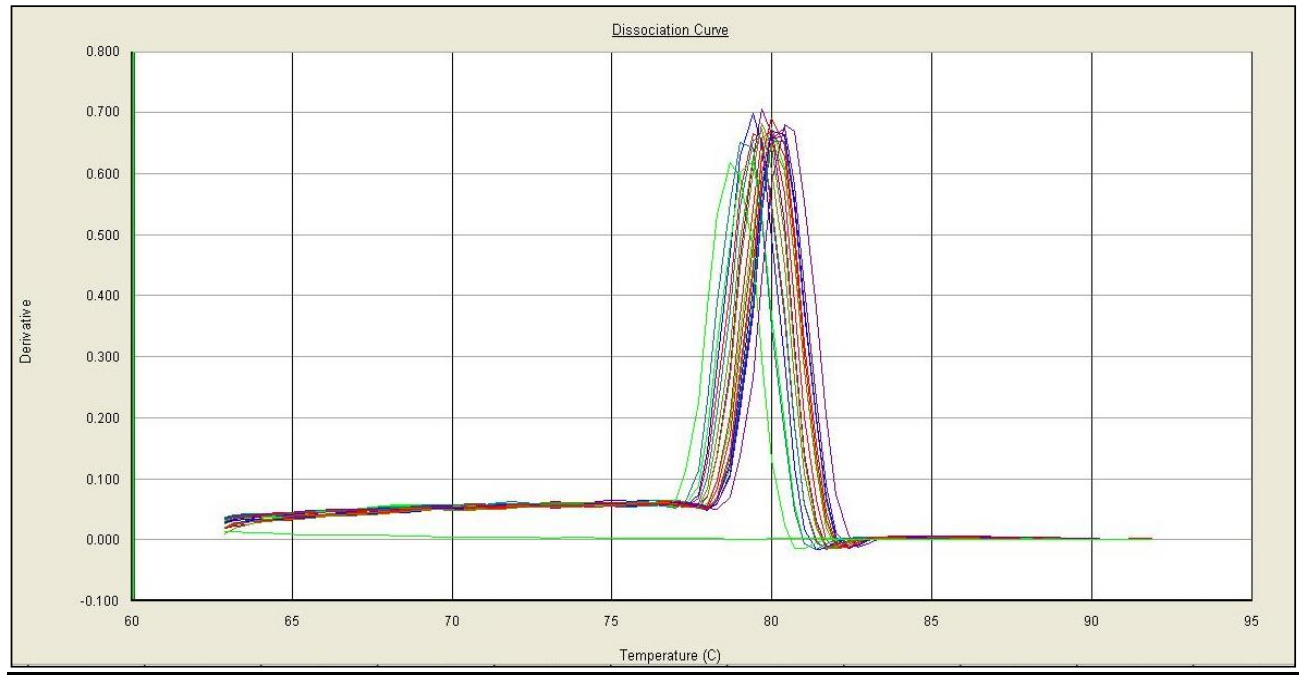

Figure 19: Dissociation curve for PECAM from 7500 Real-Time qPCR System.

$R A G E$ - From the amplification curve in Figure 20, it was found that a well corresponding with a HUVEC sample treated with $10.5 \mathrm{mM}$ glucose and a well corresponding with a HUVEC sample treated with $25.5 \mathrm{mM}$ glucose had inadequate amplification. These same wells also showed a non-desired product formed in the dissociation curve in Figure 21, and these data points were removed from further analysis. Otherwise, there was adequate amplification efficiency and amplification of a single, desired product without primer dimer formation for the remaining samples and single. The NTC showed an appropriate lack of amplification and corresponding lack of PCR product. 


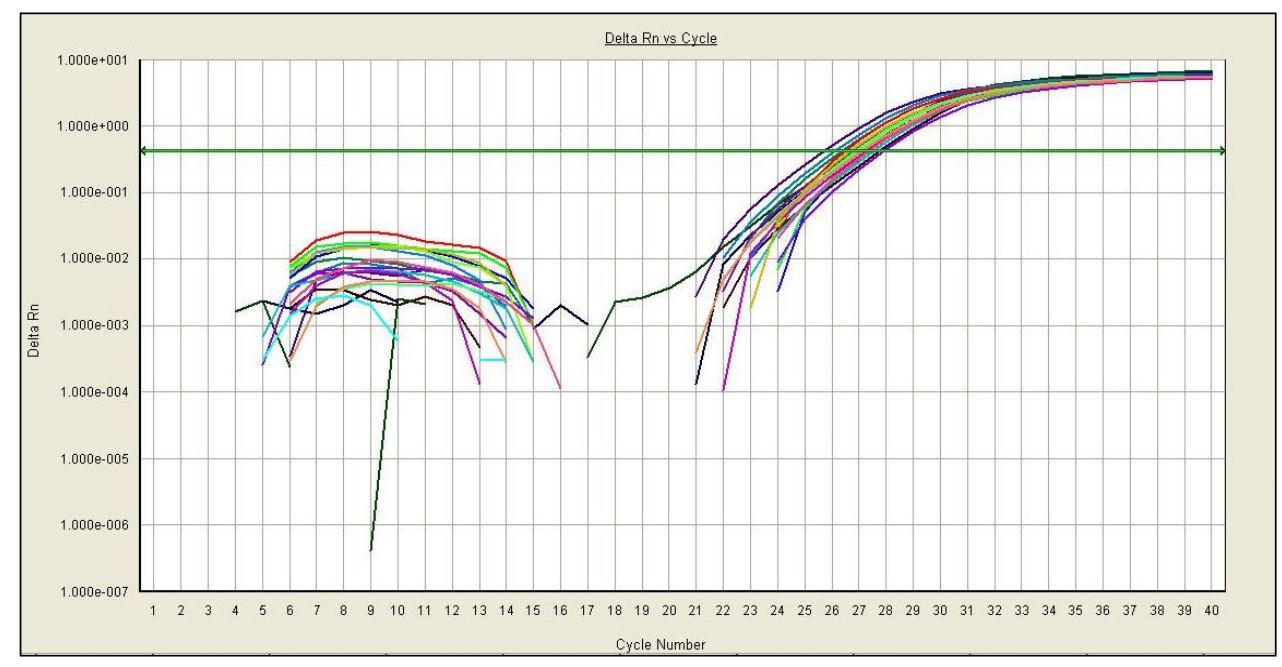

Figure 20: Amplification curve for RAGE from 7500 Real-Time qPCR System.

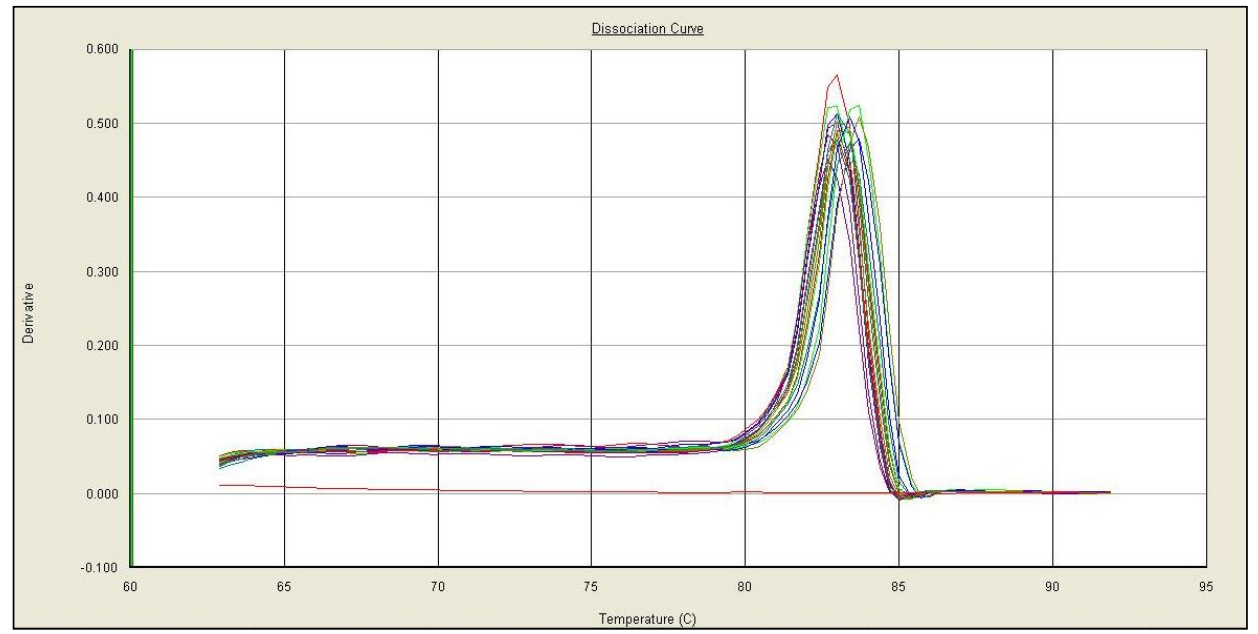

Figure 21: Dissociation curve for RAGE from 7500 Real-Time qPCR System.

eNOS - All sample wells displayed adequate PCR efficiency on the amplification curve in

Figure 22, as well as the formation of a single PCR product and lack of primer dimer formation on the dissociation curve in Figure 23. 


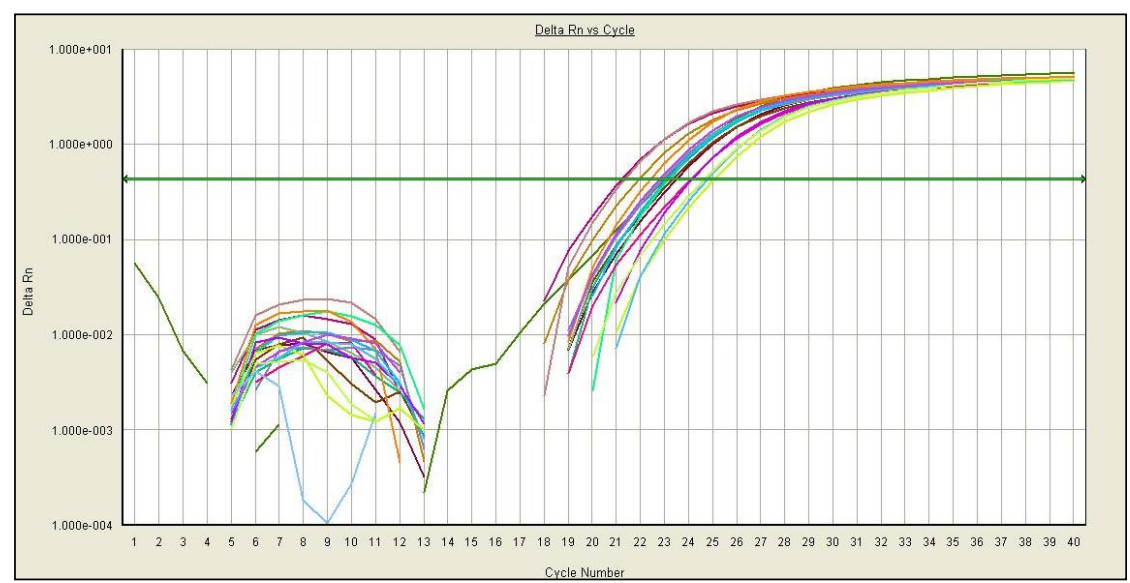

Figure 22: Amplification curve for eNOS from 7500 Real-Time qPCR System.

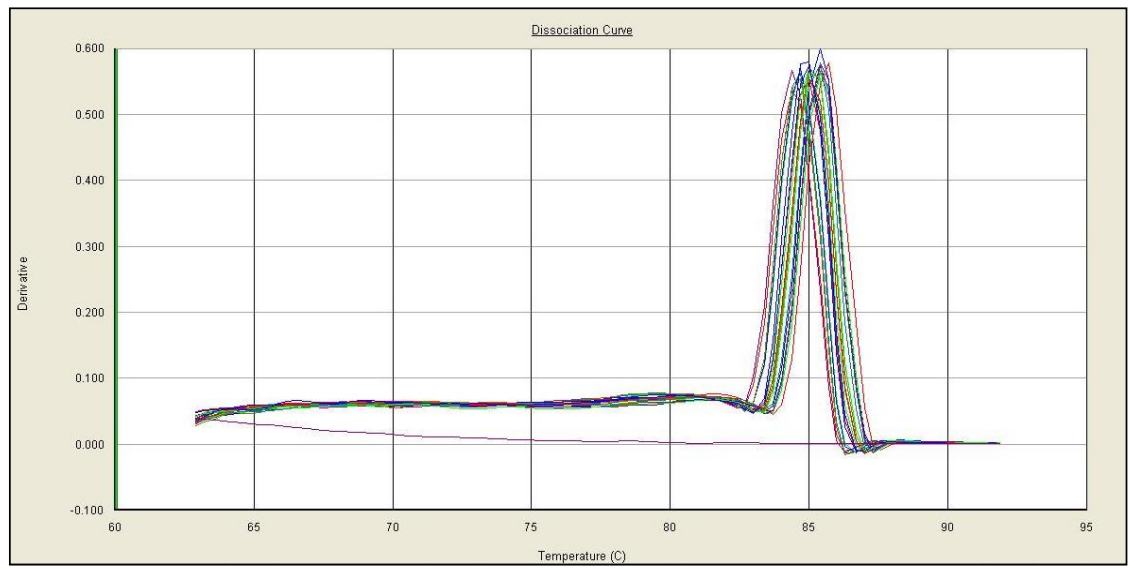

Figure 23: Dissociation curve for eNOS from 7500 Real-Time qPCR System.

The relative mean abundance \pm standard mean error is presented with significant differences determined using two-sample t-tests with unequal variance for each treatment group. In order to draw conclusions between glucose treatments as well as cell types, the data is presented for each gene with comparisons between glucose treatments within the same cell type and between two cell types with the same glucose treatment. 


\subsubsection{Comparisons Between Glucose Concentrations}

PECAM - The gene expression analysis of PECAM revealed no significant differences between the glucose concentration treatment groups within each cell type in Figure 24; however, there is a trend that PECAM expression appears to increase in the groups treated with the high glucose concentration compared to the groups did not receive any supplemental glucose within each cell type.
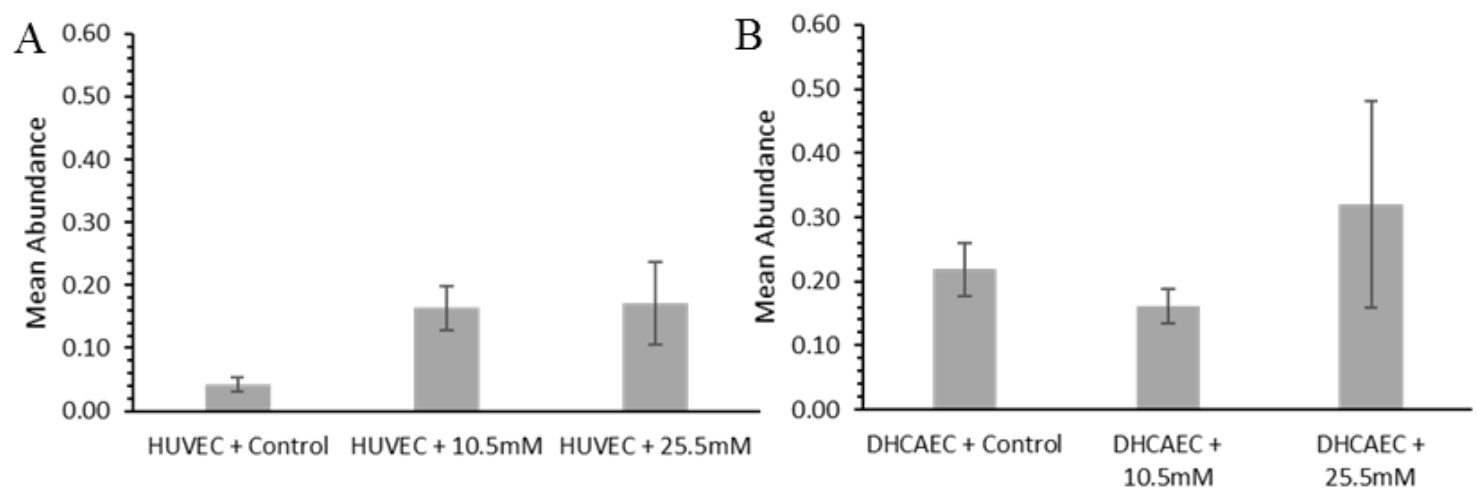

Figure 24: Results of average relative abundance of PECAM in mRNA isolated from HUVECs (A) and DHCAECs (B).

$R A G E$ - The gene expression analysis of $R A G E$ revealed no significant differences between glucose concentration treatment within each cell type in Figure 25; however, it revealed that the expression of $R A G E$ trended upward with increased glucose concentration in the cell media within each cell type. 

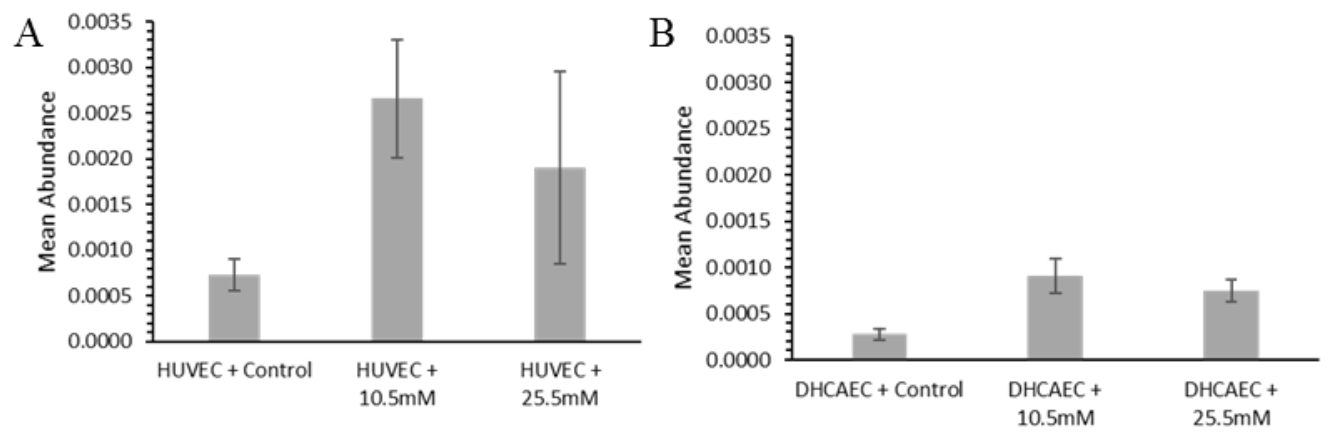

Figure 25: Results of average relative abundance of $R A G E$ in mRNA isolated from HUVECs (A) and DHCAECs (B).

eNOS - Gene expression analysis of eNOS revealed a significant difference between the HUVECs cultured in control media and 10.5mM glucose media. This difference displays a marked increase in $e N O S$ expression with increased glucose concentration. A similar trend is seen in the DHCAECs, but it was not found to be significant. HUVECs appeared to have an overall increase in $e N O S$ expression with increases in glucose concentration without significance between groups. eNOS expression did not have any noticeable trend with respect to glucose concentration in DHCAECs.
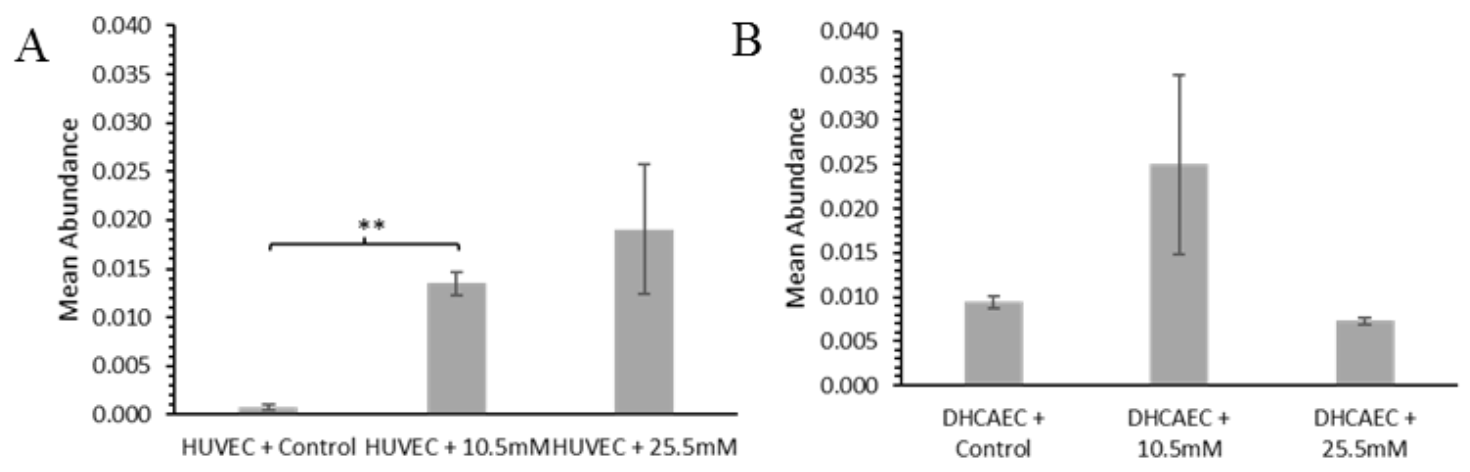

Figure 26: Results of average relative abundance of eNOS in mRNA isolated from HUVECs (A) and DHCAECs (B). 


\subsubsection{Comparisons Between Cell Type}

PECAM - The gene expression analysis for PECAM showed a significant difference in mean abundance between HUVECs and DHCAECs grown in normal cell media in Figure 27A. PECAM expression differs greatly between the two cell types when grown in $25.5 \mathrm{mM}$ glucose; however, there was no noticeable difference in PECAM expression between the two cell types when grown in $10.5 \mathrm{mM}$ glucose media.
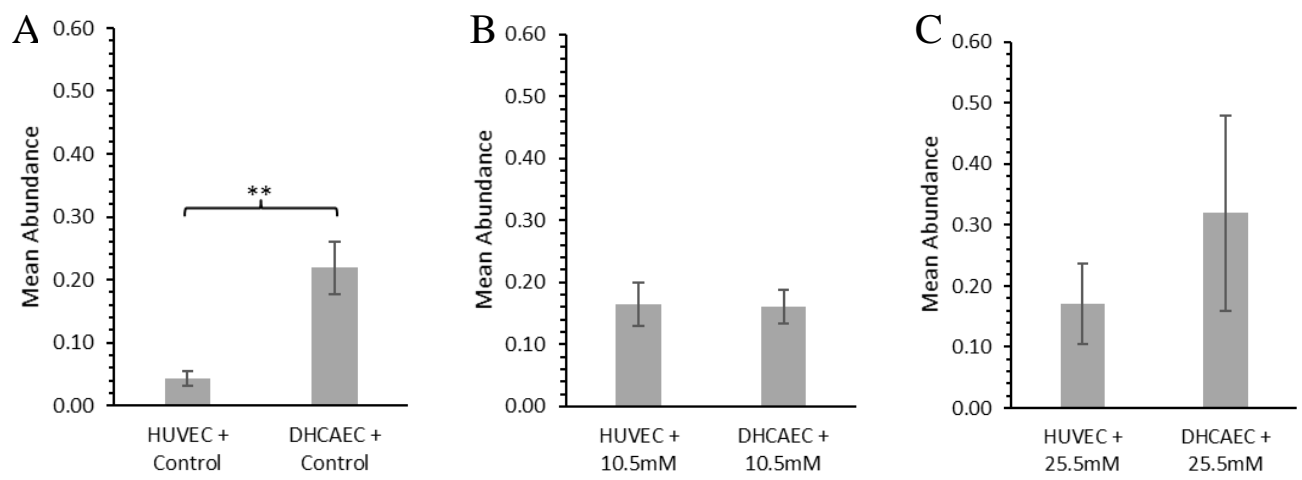

Figure 27: Results of relative abundance of PECAM in mRNA for normal glucose (A), moderate glucose (B), and high glucose (C) concentrations.

$R A G E$ - The gene expression analysis for $R A G E$ did not reveal any significant differences based on cell type; however, there was a noticeable trend. There was a trend of the HUVECs to express increased RAGE compared to the DHCAECs cultured in each respective glucose condition seen in Figure 28. 

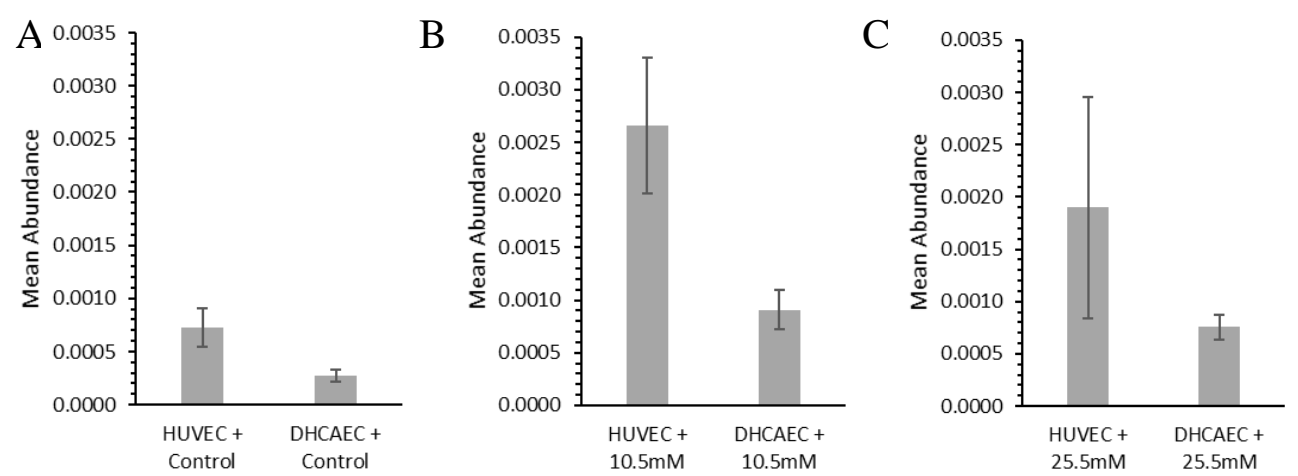

Figure 28: Results of relative abundance of $R A G E$ in mRNA for normal glucose (A), moderate glucose (B), and high glucose (C) concentrations.

eNOS - The gene expression comparison for cell type returned a significant difference between HUVECs and DHCAECs cultured in normal media as seen in Figure 29A. There were no noticeable trends associated with cell type comparison for the groups that were treated with supplemental glucose as seen in Figure 29B, C.
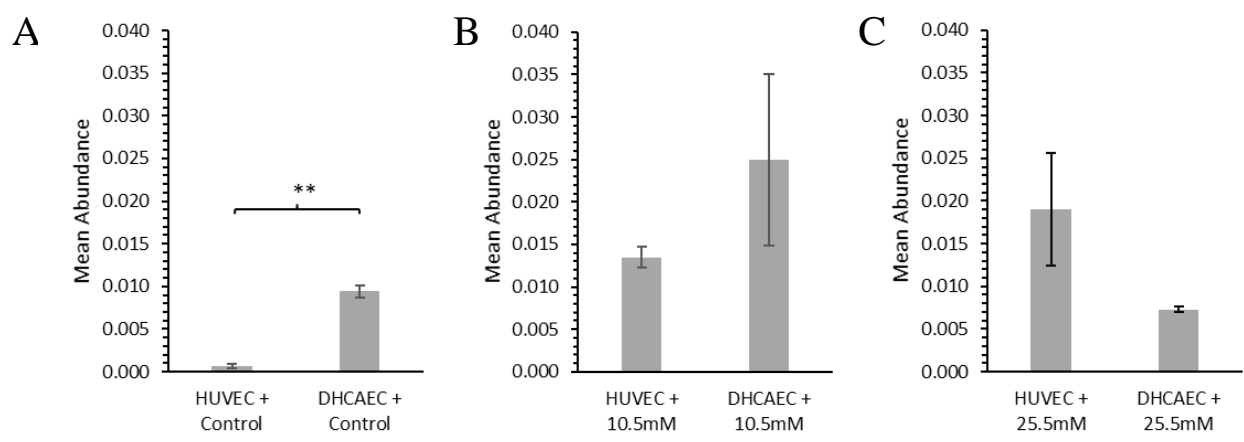

Figure 29: Results of relative abundance of eNOS in mRNA for normal glucose (A), moderate glucose (B), and high glucose (C) concentrations.

\subsubsection{Reference Gene Selection}

To determine if GAPDH was the appropriate reference gene for these samples, the $\mathrm{Ct}$ values for $G A P D H$ were transformed to linear related data using $2^{-\mathrm{Ct}}$. Then, these values 
were used in an analysis of variance (ANOVA) of GAPDH presence in each cell typetreatment combination. The data met the assumptions needed for ANOVA: the data was approximately normally distributed, the data point measurements were independent of one another, and had approximately equal variance. It was found that the variance of each treatment group was not significantly different from one another.

\subsection{Discussion}

The immunofluorescence images obtained from this preliminary study did not reveal as much information as anticipated. Due to the subjectivity of determining PECAM expression from the immunofluorescence images, the analysis was largely qualitative. The BBI images suggested that confluency decreased as glucose concentration increased for DHCAECs (Figure 15A, D, G), which may indicate apoptosis of cells in culture. From the PECAM images alone, it appeared that all samples had some PECAM-positive staining, demonstrating that the cells present are most likely endothelial cells. Unfortunately, a live-dead stain was not performed on the samples for this study, which would have allowed for the determination of apoptotic activity in endothelial cells grown in an in vitro hyperglycemic environment.

From the gene expression analysis, there were few significant differences in expression of any of the genes of interest due to the addition of glucose to the cell media; however, there were several noticeable trends relative to glucose concentration in the media. One possible reason for the lack of significance in statistical analysis is the relatively large standard error values. Standard error accounts for variability relative the sample size. In this study, the largest source of error in gene expression can be attributed 
to pipetting error when adding samples and reagents to plate for PCR. Another possible source of error is the small sample size ( $\mathrm{n}=2$ or 3 ) for all samples.

With the trends seen in the relative quantities of each gene, the moderate glucose concentration $(10.5 \mathrm{mM})$ treatment groups were largely inconsistent. For PECAM, there appeared to be a moderate decrease in expression for DHCAECs cultured in $10.5 \mathrm{mM}$ glucose media compared to the control and the $25.5 \mathrm{mM}$ glucose media. This resulted in virtually no difference in expression between cell types at this glucose concentration for PECAM. The $10.5 \mathrm{mM}$ glucose media groups were inconsistent with the trends for both cell types for RAGE expression, and for DHCAECS for eNOS expression. This may be attributed to the fact that $10.5 \mathrm{mM}$ of glucose is relatively conservative concentration to represent a hyperglycemic environment, as concentrations nearing $10 \mathrm{mM}$ are considered representative of blood glucose levels associated with diabetes [31]. Due to the complexity of the diabetic disease process, this conservative glucose level may not prompt the same responses in either HUVECs or DHCAECs as expected in a hyperglycemic environment. Another possibility for these inconsistencies may stem from the production of the glucose solution used to supplement cell media. This solution was prepared from $18 \mathrm{~m} \Omega$ water and solid glucose (Sigma Aldrich, St. Louis, MO). This process has a likelihood of human error throughout the process of weighing and combining the glucose with water. The concentration for the glucose solutions were most likely different from the theoretical values used to perform the calculations. There were also challenges with sterilizing this glucose solution, including determining how to sterilize the solution. Ultimately, the glucose solution had to have a low enough concentration such that when sterilized in the autoclave, the glucose did not burn; 
however, heating solution to past the boiling point of water could alter the volume which may also have contributed to error in glucose concentrations. This source of error can be minimized, or even eliminated, through the use of sterile glucose solution acquired from a reliable source that has been analyzed to ensure accurate concentration values.

Limitations of this preliminary study include the static, 2-dimensional nature of the culture conditions, as well as the minimal concrete data attained from immunofluorescence analysis. Ideally, the immunofluorescence observations could be used to compare the phenotypic response versus the nuclear, mRNA, response of endothelial cells exposed to high glucose culture conditions. In this preliminary study, it was found that immunofluorescence data is not specific or sensitive when imaged using widefield fluorescence microscopy; however, due to time constraints and available resources, improved images could not be acquired from confocal microscopy.

The goal of the tissue engineering lab at Cal Poly is to produce a blood vessel model that accurately represents certain aspects of blood vessels in vivo. This preliminary study was conducted on cells cultured in static conditions in a 2-dimensional monolayer. First, the static nature of these culturing conditions may prevent the equal distribution of glucose through the cell flask. Once all the local glucose is consumed, there are no means for the redistribution of media once cells are fed or passed, resulting in a local environment that may not continue to have an elevated glucose concentration. This may result in skewed gene expression data as glucose may no longer be present to activate the processes persistent in diabetes. In future studies, this may be addressed by continually monitoring glucose concentration in the cell flasks and in the bioreactor and adding 
sterile glucose solution as necessary to maintain the desired levels of glucose concentration.

Secondly, it has been established that 3-dimensional (3D) culturing systems are superior to 2-dimensional (2D) culturing systems due the ability of 3D cell culturing to better replicate physiological systems and provide more predictive data for in vivo tests [60]. A key difference between the 2D and 3D culture conditions for the study of diabetes in vitro is the differences in cellular responses due to the spatial organization of the cell surface receptors (i.e. PECAM) that take part in cell-cell and cell-extracellular matrix (ECM) interactions. The physical interactions between cells with each other, the ECM, and their microenvironment in 3D culture affects cellular functions, including cell proliferation, differentiation morphology, and protein and gene expression [60]. The preliminary study was performed using $2 \mathrm{D}$ culturing conditions; however, to better mimic in vivo conditions in vitro the final study was completed with $3 \mathrm{D}$ culture conditions.

\subsection{Conclusion}

The aim of this preliminary study was to determine an optimal glucose concentration to use in the construction of a diabetic BVM. This study was also used to verify the updated qPCR protocol that was originally established for the lab. This preliminary study utilized two methods of analysis to establish a protocol for the implementation of a high glucose cell media for culturing endothelial cells to be cultivated in BVMs. Although immunofluorescence is commonly used in the tissue engineering lab for analysis of completed BVMs, this study utilized this analysis with disappointing results. There were 
numerous drawbacks of immunofluorescence, including that only qualitative, subjective observations could be made regarding the presence of PECAM. The utility of immunofluorescence is better observed in studies identifying multiple cell types or the spatial organization in a more complex system, like a BVM. However, the use of immunofluorescence confirmed the presence of cells at the completion of treatment, demonstrating the feasibility of adding glucose to the cell media without causing extensive apoptosis.

Gene expression analysis demonstrated significant differences due to cell type, and - to a small extent - glucose concentration in cell media. The application of the gene expression analysis protocol was successful, as observed by the procurement of quality data. The analysis of this data revealed trends in gene expression that were not expected; however, potential sources of error were identified along with changes that were applied to the following experiment in chapter 3 . These changes included using a sterile glucose solution with a known and verified concentration, and eliminating the $10.5 \mathrm{mM}$ glucose treatment. The $10.5 \mathrm{mM}$ was originally chosen as a conservative increase in glucose concentration because it was unknown how well the HUVECs and DHCAECs would survive in the elevated glucose media treatments. From the preliminary study, there were irregularities found in the gene expression data for this treatment group and the determination that this concentration may not accurately reflect hyperglycemic glucose levels in vivo. For further studies, this treatment was removed.

The next chapter discusses the expansion of this study to include the 3D culturing of HUVECs and DHCAECs, grown in media with elevated glucose concentrations, in a BVM setup. A BVM study was performed to further characterize aspects of the diabetic 
blood vessel model. To increase the sample size for more accurate gene expression analysis, biological replicates of each cell type and treatment combination were included in addition to technical replicates for each qPCR. 


\section{Chapter 4: Applying High Glucose Conditions to HUVECs and DHCAECs in Tissue Engineered Blood Vessel Mimics}

\subsection{Introduction}

In chapter 2 it was established that there are significant differences in gene expression for healthy (HUVECs) and diabetic (DHCAECs) endothelial cells cultured in vitro, as well as for some of the different levels of glucose concentration. The preliminary study also demonstrated that the DNA primers chosen for qPCR analysis were satisfactory and functional in the application of the protocol for gene expression analysis.

As discussed in chapter 3, the results from gene expression analysis were not as expected; however, it was hypothesized that by introducing the cells into a dynamic 3dimensional model these results may change and more accurately reflect the proposed disease processes that occur in endothelial cells in diabetes. The blood vessel mimic construct somewhat more accurately reflects anatomical geometry and shear forces experienced in vivo as compared with the 2-dimensional, static culture used in the preliminary study. Based on previous studies comparing differences in 2D and 3D culture conditions, it is shown that 3D culture conditions better replicate physiological systems and provide more predictive data for in vivo tests [60]. Due to these findings, the final study was completed using 3D culture conditions using the bioreactor set up for blood vessel mimics to best replicate in vivo physiologic conditions that may affect gene expression and cell proliferation.

The same analysis methods were used on BVMs as in the preliminary study, with the addition of SEM imaging. SEM imaging has been used previously to evaluate the morphology of the endothelial lining in the lumen of BVMs [61]. 


\subsection{Materials and Methods}

HUVECs and DHCAECs were initially cultured and expanded using the same protocol described in chapter 2 . To avoid any complications that may have been due to the glucose solution used in the preliminary study, 20\% w/w $(1.1935 \mathrm{M})$ sterile glucose solution (AMRESCO, E545-100ML, Solon, OH) was used to produce cell media with an elevated glucose concentration of $25.5 \mathrm{mM}$ by adding $8.56 \mathrm{~mL}$ of glucose solution to $500 \mathrm{~mL}$ of prepared endothelial cell media. The glucose treatment was introduced to cells in the T225 culture flasks 6 days prior to application of cells to BVM culturing conditions. Once the cells were introduced to the BVM culture conditions, the glucose treatment was continued using bioreactor media - with $9.750 \mathrm{~mL}$ of $20 \%$ w/w sterile glucose solution added to $569.4 \mathrm{~mL}$ of media for the high glucose condition.

\subsubsection{BVM Set Up}

Two groups of 6 BVMs were cultivated at a time due to the number of sterilized bioreactor chambers available. The first group consisted of DHCAECs at passage 5 at approximately $90 \%$ confluence in T225 flasks. One T225 was used per BVM construct. This first set of BVMs contained 3 BVMs treated with high glucose $(25.5 \mathrm{mM})$ cell media and 3 BVMs cultivated with normal (5.5mM glucose) cell media. The second group of BVMs consisted of HUVECs at passage 6 at approximately 95\% confluence in T225 flasks, with one flask used per BVM. Due to time constraints and the HUVECs not reaching confluency at passage 5 in three T225 cell flasks, the HUVECs were unable to be passed into six T225 cell flasks before the glucose treatment needed to be introduced to maintain the same time frame -6 days prior to BVM introduction - as the DHCAEC 
group of BVMs. Therefore, the high glucose treatment was introduced to two of the T225 flasks of HUVECs and the remaining T225 flask of HUVECs was treated with the control glucose media. After the glucose treatment was introduced, the three T225 flasks of HUVECs were passed at a 1:2 ratio to obtain the six T225 cell flasks needed for BVM set up. This resulted in the second set containing 4 BVMs cultivated in high glucose cell media and 2 BVMs cultivated in normal cell media.

The methods used to cultivate BVMs have been previously developed and described [62]. In summary, 75:25 PLGA electrospun scaffolds were mounted to fittings and sterilized in $70 \%$ EtOH for 30 minutes. The scaffolds were then flushed with DCFPBS, followed by conditioning media. Scaffolds were then mounted on fittings in an ethylene oxide sterilized chamber, which allowed for the connection to a circuit of tubing. This circuit connected the chamber to a reservoir of media and passed through an 8-roller peristaltic pump to provide flow of media through the circuit. Conditioning media was pumped through scaffolds for approximately 24 hours prior to sodding of cells.

For this experiment, normal conditioning media was used for all scaffolds. Once conditioning was completed, the media in the chamber and reservoirs was exchanged for the appropriate cell media for each treatment group.

\subsubsection{Take Down and Evaluation}

Three days after sodding cells in the BVM construct, the BVMs were removed from the bioreactor chamber and gently flushed with DCF-PBS. Approximately $1 \mathrm{~cm}$ of the BVM was cut transversely from the proximal portion and fixed in $10 \%$ formalin, and 
used for immunofluorescence. The remainder of the BVM was cut longitudinally. For each treatment group, one longitudinal section was fixed in $2.5 \%$ glutaraldehyde, dried in ethanol solutions, placed in a desiccator for 24 hours and used for SEM analysis. En face SEM images were obtained for one biological replicate of each treatment group. The SEM images were obtained using a standardized protocol.

Immunofluorescence samples were removed from formalin and embedded in TissueTek OCT and frozen quickly using liquid nitrogen. These blocks were sectioned transversely using a cryostat maintained at $-20^{\circ} \mathrm{C}$. Cross-sections $20 \mu \mathrm{m}$-thick were obtained and stored on slides at $-20^{\circ} \mathrm{C}$ prior to staining. These samples were stained using the protocol previously discussed in chapter 3.

The remainder of the longitudinal BVM was rinsed with DCF-PBS to remove remaining cell media, and used immediately for gene expression analysis via qPCR. BVM sections used for gene expression were rinsed a second time with DCF-PBS and then placed in a lysis buffer and vortexed for 2 minutes to release cell contents and homogenize cells in the BVM. This cell lysate was then used to isolate RNA as previously described in chapter 2 . The isolated RNA was assessed, and used in reverse transcription to obtain cDNA using the appropriate volume for each RNA, determined by equation 2.1 and the data presented in appendix L. Complementary DNA obtained for each biological replicate was evaluated with real-time qPCR following the same specifications as the preliminary study. Due to the number of samples obtained, two plates were needed to run all samples, shown in the plate templates in appendix $\mathrm{J}$. 


\subsubsection{Statistical Analysis}

The evaluation of gene expression was determined using the same methods described in the preliminary study. Due to the addition of biologic replicates for each experimental group, the mean abundance data included all biologic and technical replicates for each experimental group.

\subsection{Results}

The production of BVMs is a sensitive process that has numerous opportunities for error. Fortunately, the inclusion of biological replicates mitigated the loss of all data for any of the treatment groups. In the first group of DHCAEC BVMs, one of the BVMs cultivated in control media fell off the fitting during cell sodding resulting in the loss of cells from the construct and was not used for analysis due to the lack of cells that remained in the BVM.

\subsubsection{Immunofluorescence}

Similar to the preliminary study, a representative image is presented for each treatment group for BBI, PECAM, and a composite of the two images were created using ImageJ Software, at 10 times objective magnification.

It should be noted that the orientation of each cross-section varies, and for clarification of BVM orientation the luminal side is noted by the asterisk. In the immunofluorescence images for the HUVEC BVMs, the PECAM stain is noticeably brighter and more prevalent in the group treated with $25.5 \mathrm{mM}$ glucose media as compared to the control glucose in Figure 30. The opposite appears to be true in the 
immunofluorescence images for the DHCAEC BVMs in Figure 31, where it was noted that the group treated with control glucose media had a brighter and more widespread PECAM stain than the group treated with high glucose media. 

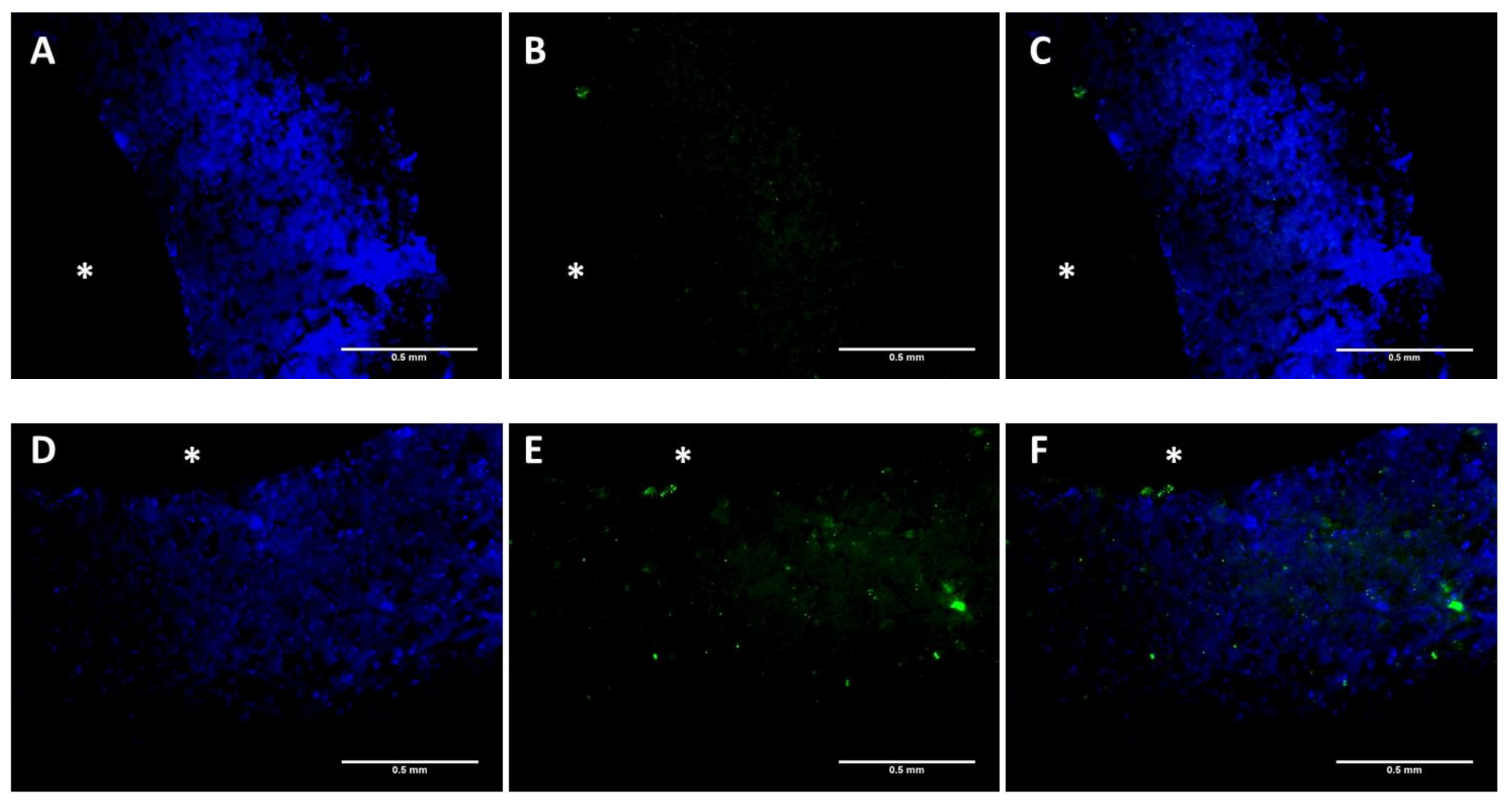

Figure 30: BVMs cultivated with HUVECs in control (A-C) and 25.5mM of glucose (D-F). (A, D) BBI staining of BVM crosssections. (B, E) PECAM staining of BVM cross-sections. (C, F) Composite image of both immunofluorescence stains. 


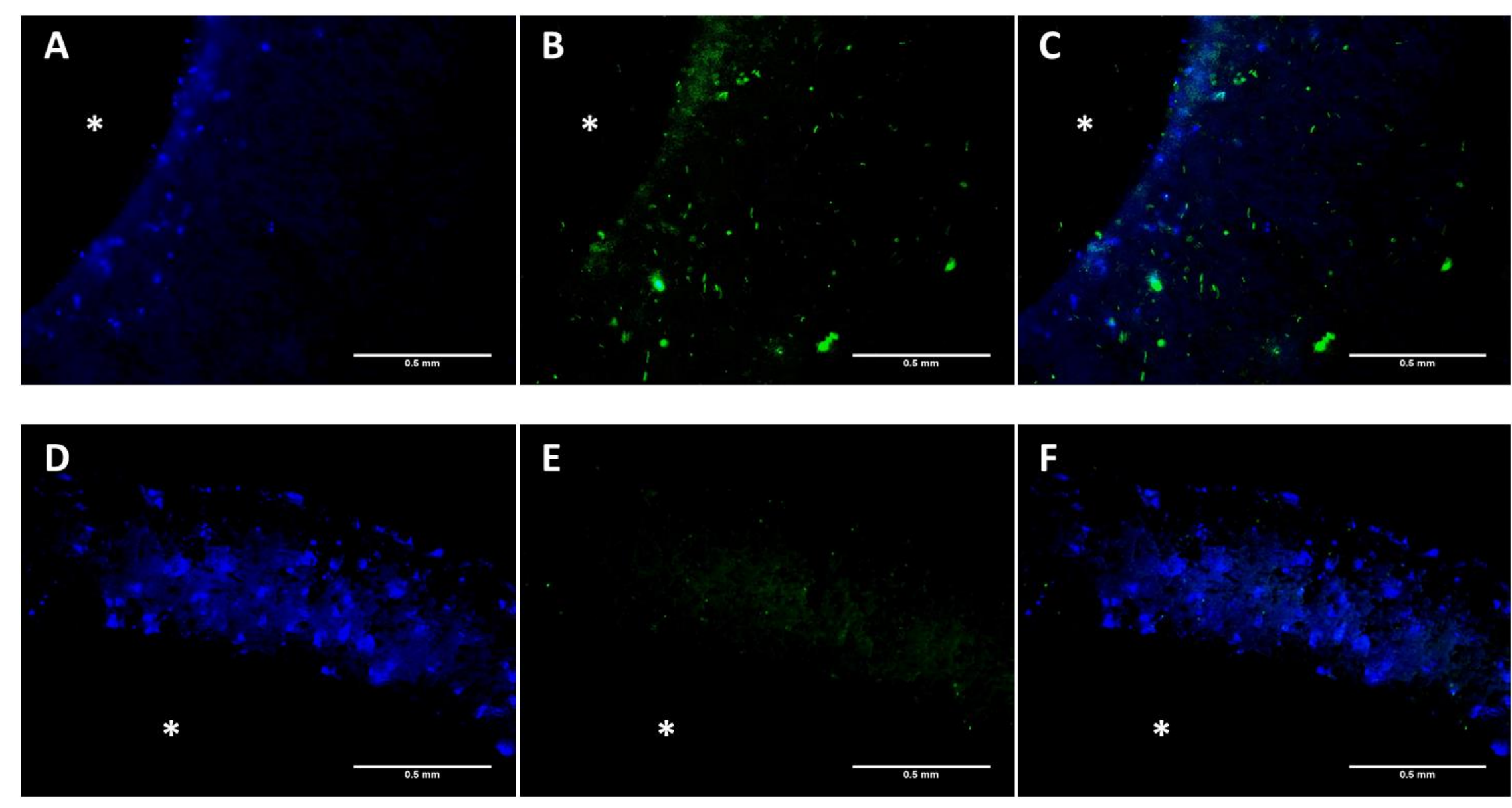

Figure 31: BVMs cultivated with DHCAECs in control (A-C) and 25.5mM of glucose (D-F). (A, D) BBI staining of BVM crosssections. (B, E) PECAM staining of BVM cross-sections. (C, F) Composite image of both immunofluorescence stains. 


\subsubsection{SEM Imaging}

A representative image is presented for each treatment group at 50x and 500x magnification. SEM images of all treatment types revealed a very minimal presence of a cell lining on the scaffold, with even less coverage in the groups treated with high glucose media compared to those in control media, as seen in Figure 32B and $\mathbf{D}$ versus $\mathbf{A}$ and $\mathbf{C}$, respectively. This is easier seen in the DHCAEC BVMs at 500x magnification images in Figure 33.
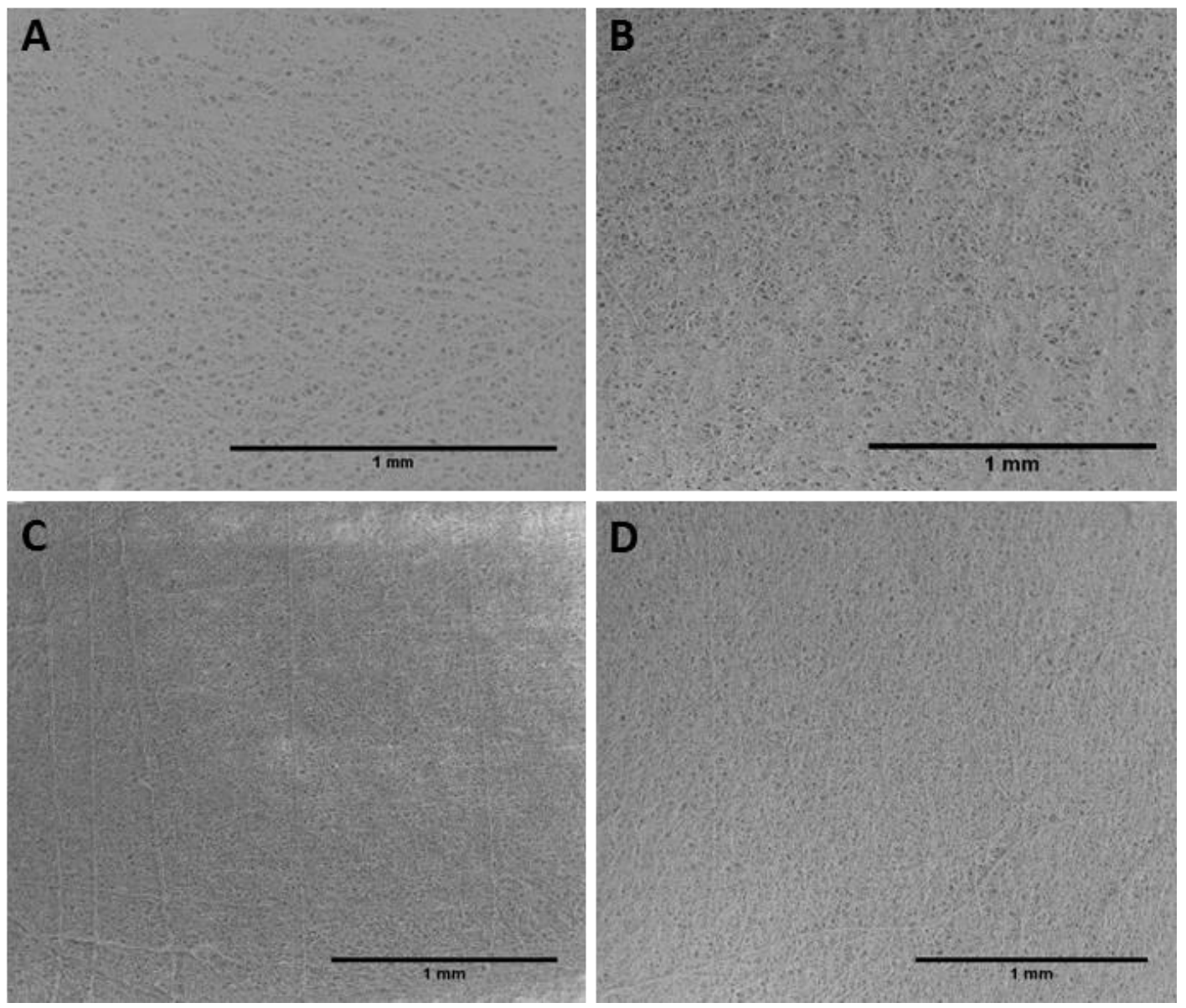

Figure 32: SEM images obtained at 50x magnification of BVMs cultured with HUVECs in control media (A), HUVECs in high glucose media (B), DHCAECs in control media (C), and DHCAECs in high glucose media (D). 

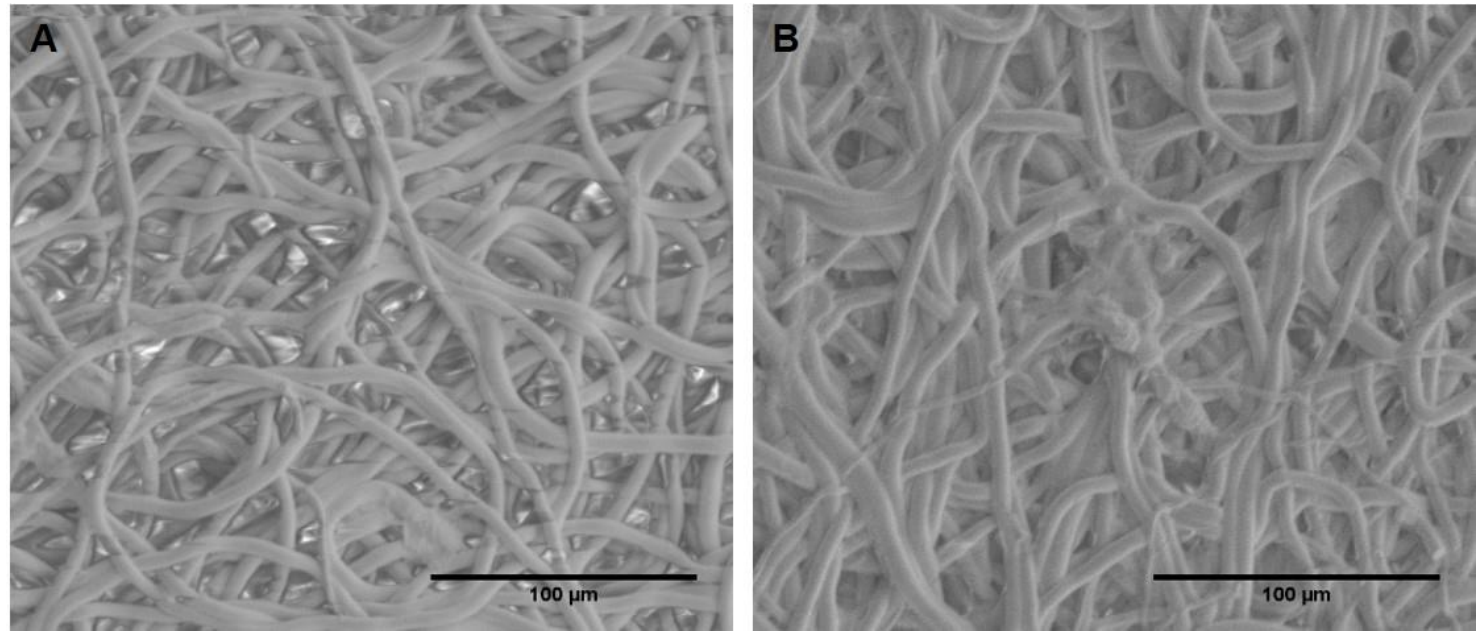

Figure 33: SEM images obtained at 500x magnification of BVMs cultured with DHCAECs in control media (A) and DHCAECs in high glucose media (B).

\subsubsection{Gene Expression}

From the RNA assessment performed prior to cDNA synthesis, it was found that a BVM from the DHCAEC group that received the elevated glucose treatment and a BVM from the HUVEC group that received the elevated glucose treatment did not have an A260/280 value within the acceptable range, and was consequently removed from further data analysis.

\subsubsection{Quality Assessment}

The amplification and dissociation curves for each qPCR run were used to verify the efficiency and products of PCR amplification. Due to the need for two plates to run all the samples, plate 1 was all samples from the first group of BVMs using DHCAECs and plate 2 was all samples from the second group of BVMs using HUVECs. 


\section{$G A P D H$}

The amplification curves, provided in Figure 34, revealed some amplification of the NTC. The NTC also had primer dimer formation that was revealed in the dissociation curves in Figure 35. One well from a BVM cultivated with DHCAECs in control media did not have adequate amplification efficiency and was removed from data analysis.

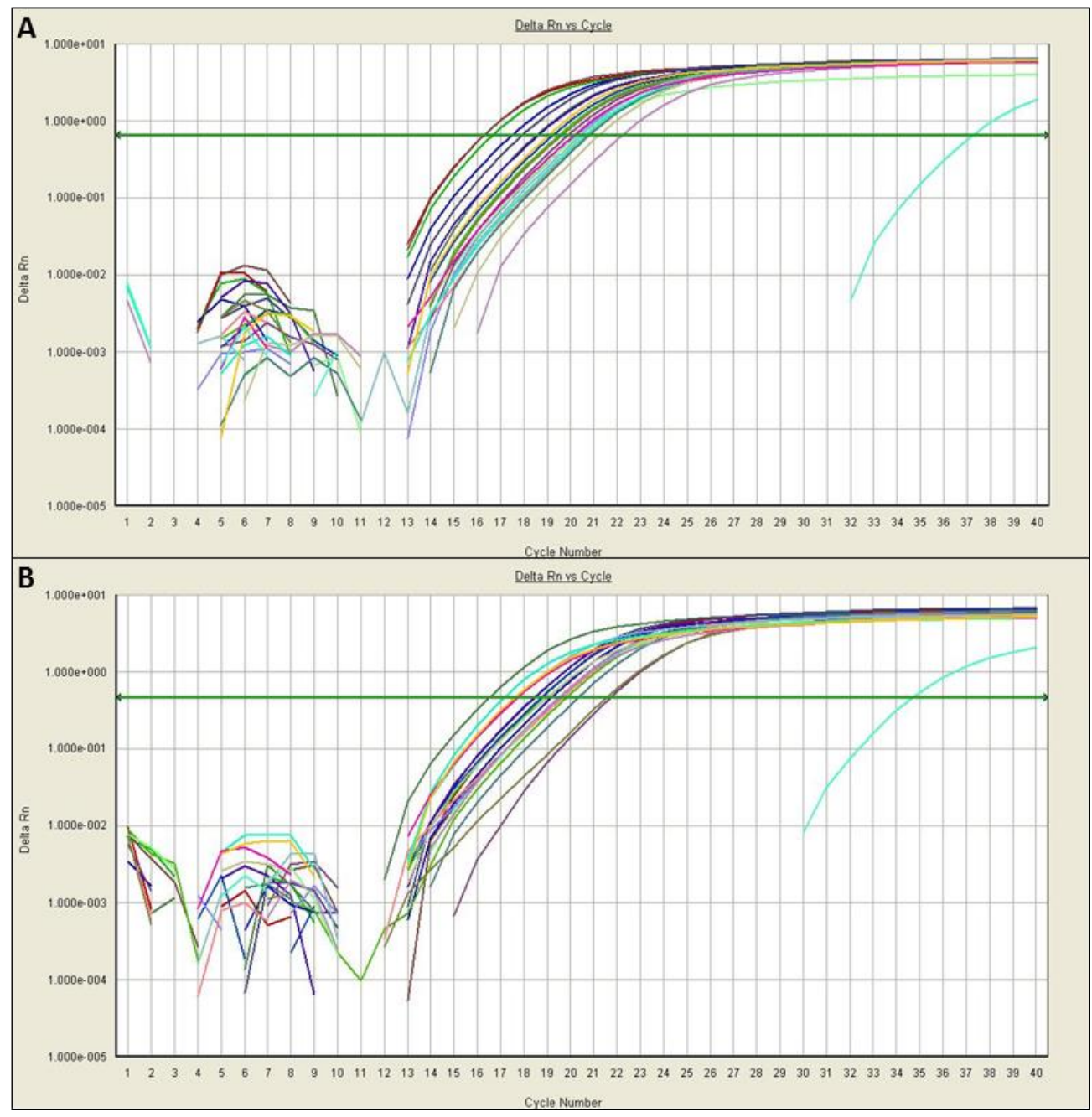

Figure 34: Amplification curves for plate 1 (A) and plate 2 (B) for GAPDH. 


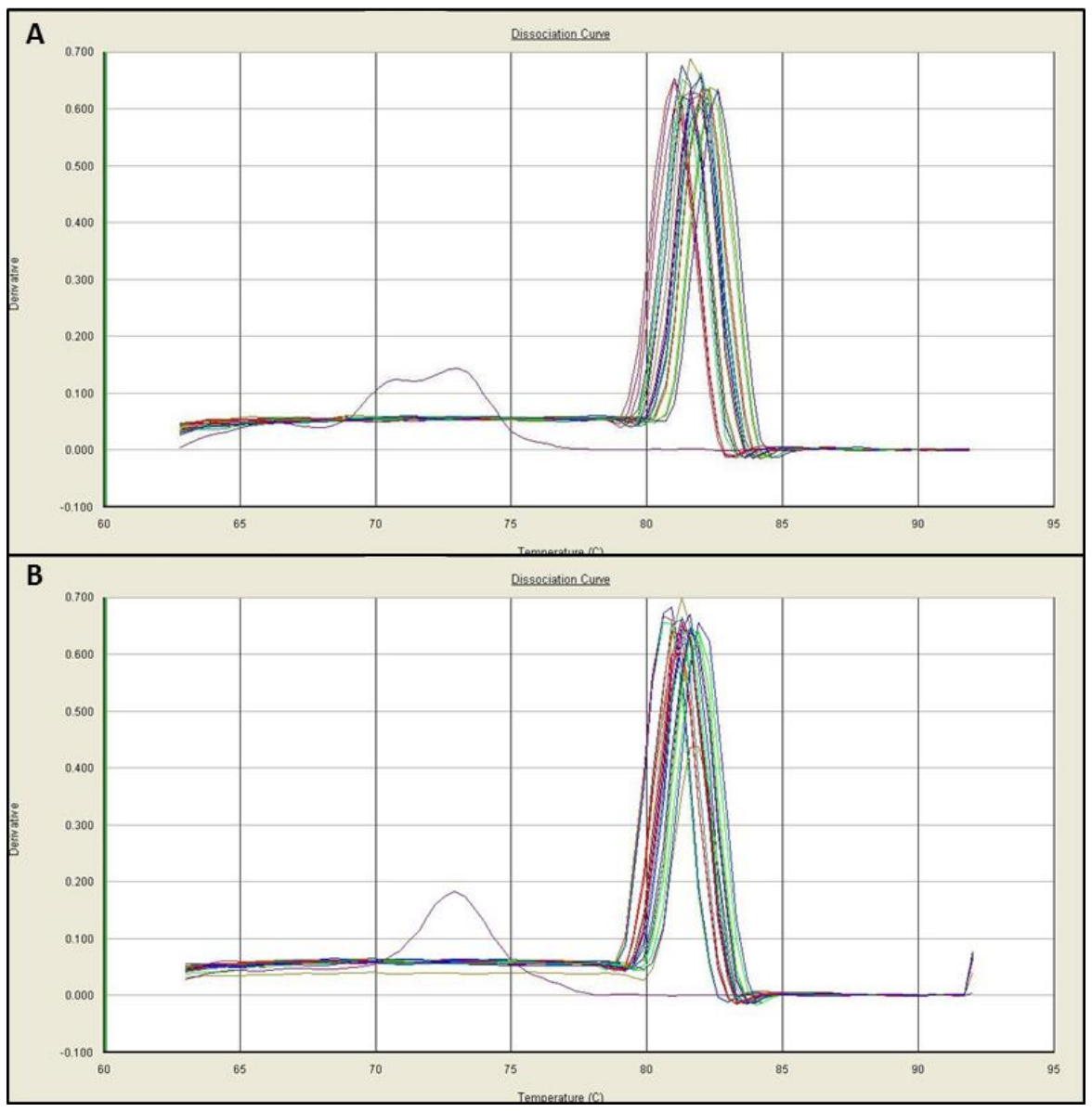

Figure 35: Dissociation curves for plate 1 (A) and plate 2 (B) for GAPDH.

PECAM - The amplification curves for PECAM, provided in Figure 36, revealed adequate efficiency for all samples except for one well associated with a BVM with DHCAECs in high glucose, two wells from a BVM with HUVECs in normal media - the control group, and one well from a HUVEC with glucose. The dissociation curves, seen in Figure 37, confirmed the poor quality of the listed wells, as well as showed a single PCR product for the remaining samples and no primer dimer formation in any sample. The data obtained from the listed wells were omitted from further analysis. 


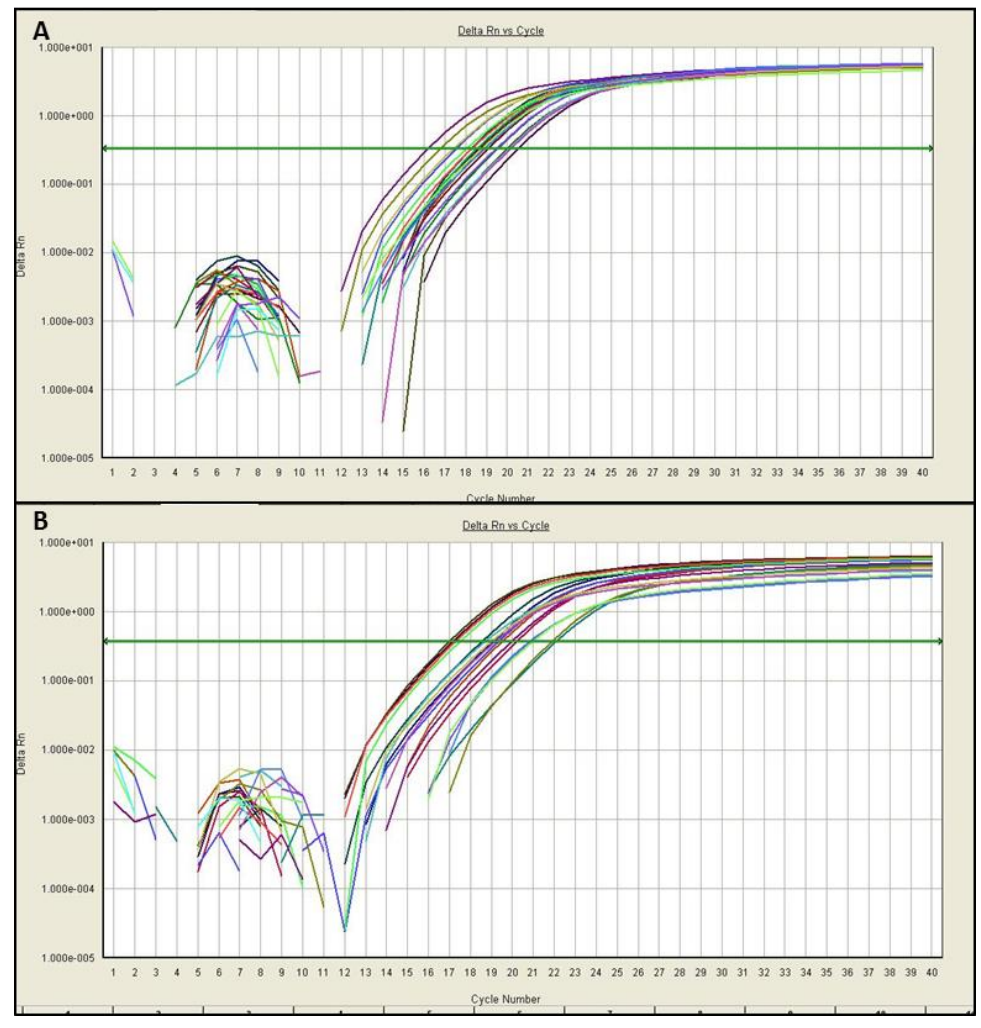

Figure 36: Amplification curves for plate 1 (A) and plate 2 (B) for PECAM.

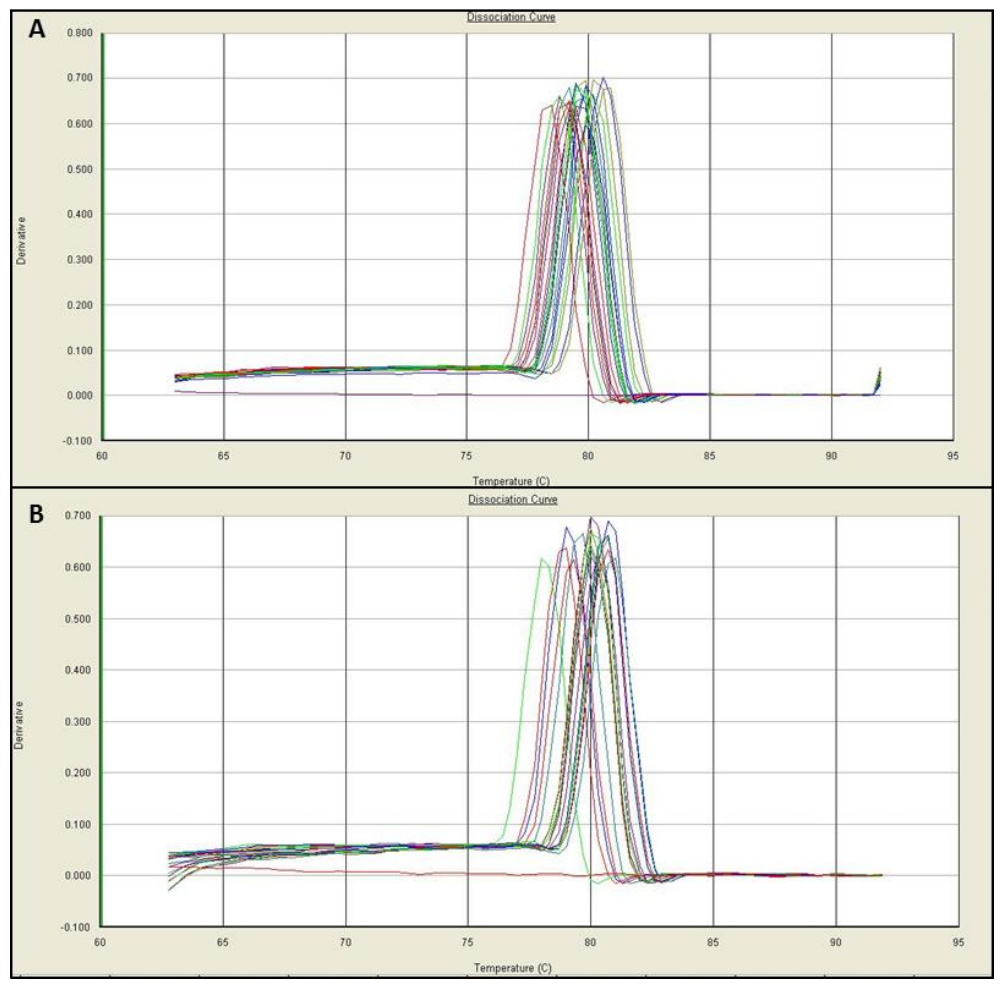

Figure 37: Dissociation curves for plate 1 (A) and plate 2 (B) for PECAM. 
$R A G E$ - The amplification curves, provided in Figure 38, showed adequate amplification efficiency with the exception of two wells associated with a HUVEC control BVM, and one well associated with a glucose treated HUVEC BVM. This observation was confirmed by the formation of non-desired PCR product in the dissociation curves, shown in Figure 39, for the listed wells and the data for these wells were subsequently omitted. The remaining wells showed formation of a single, desired PCR product.

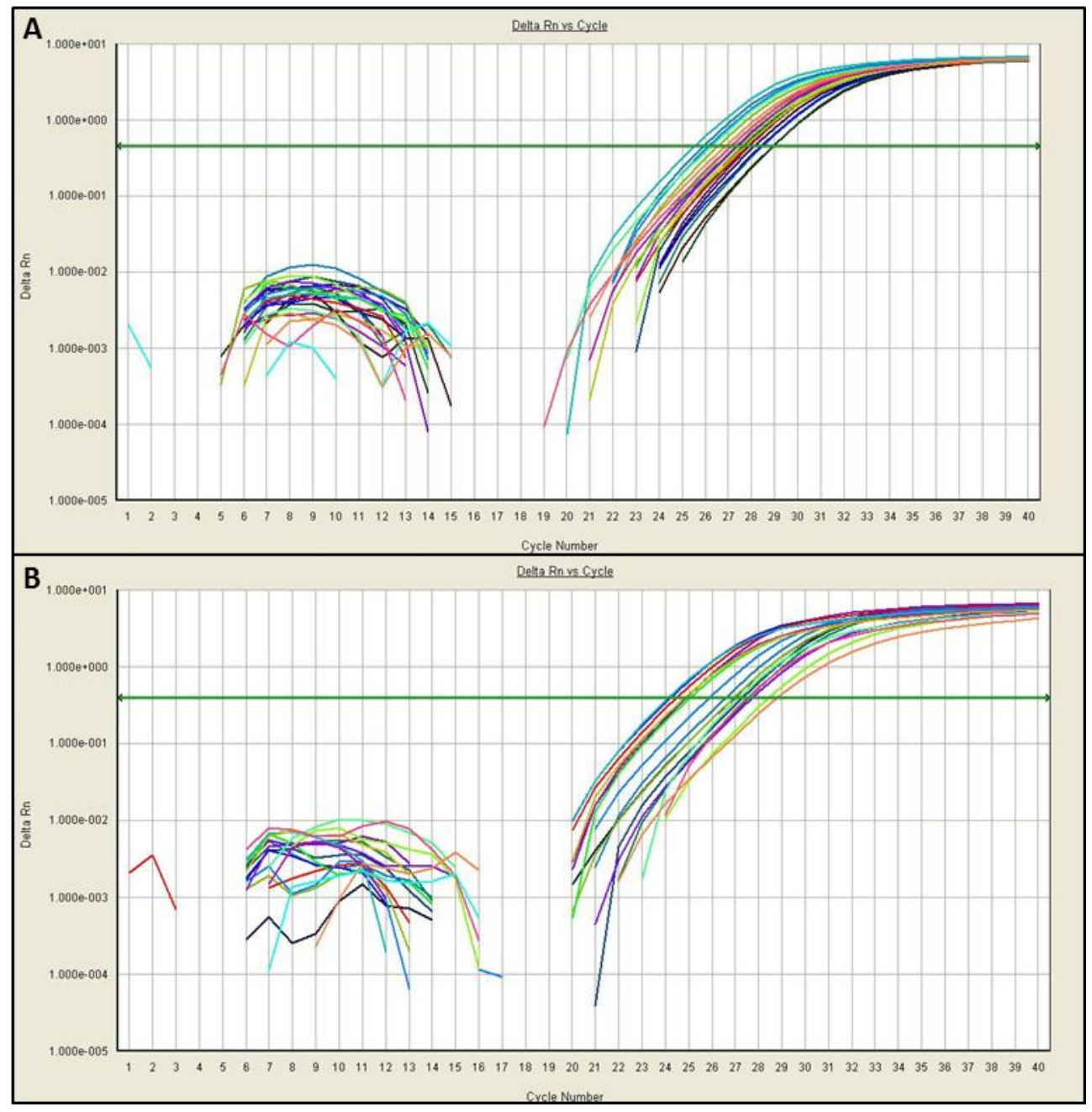

Figure 38: Amplification curves for plate 1 (A) and plate 2 (B) for RAGE. 


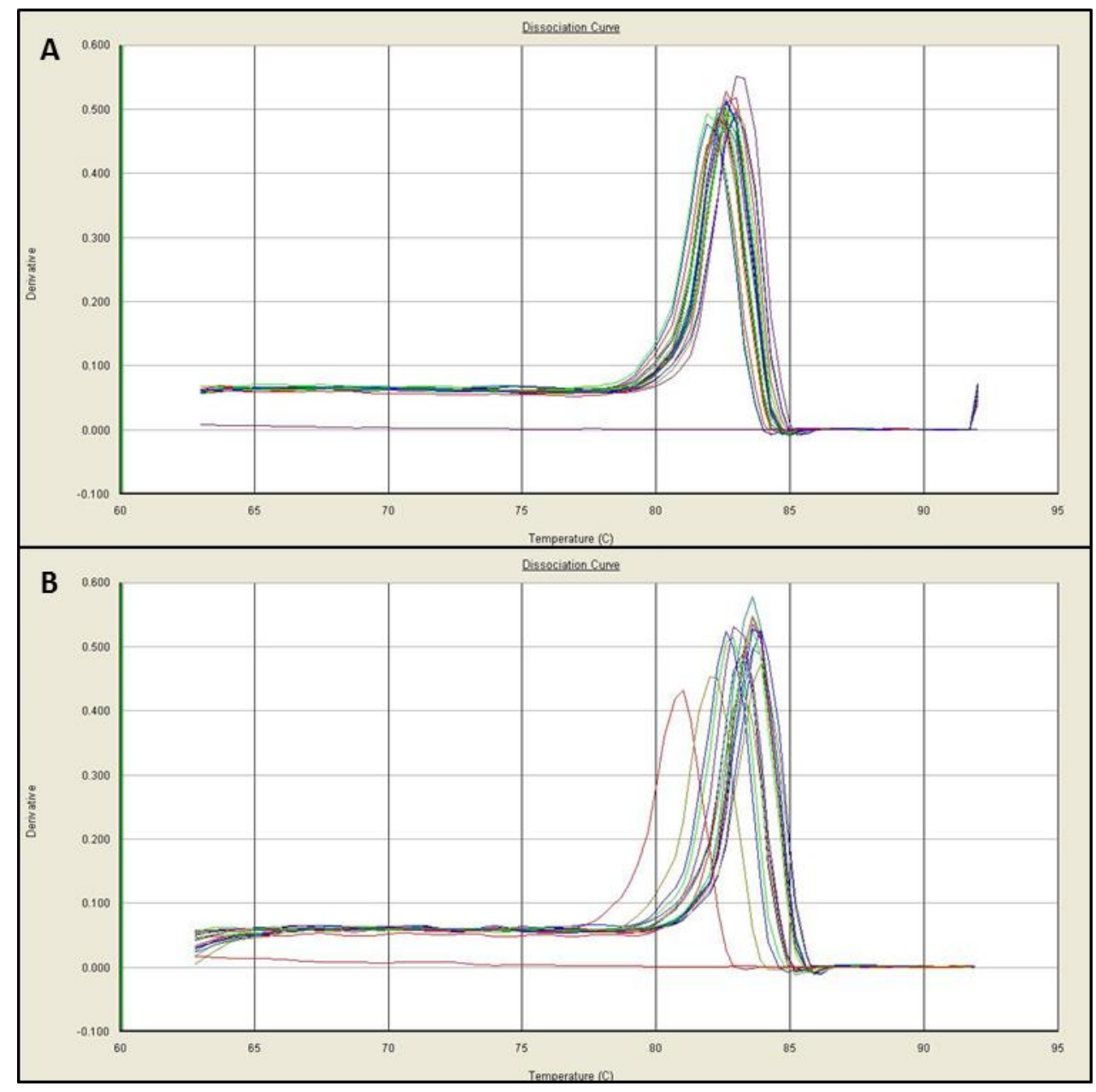

Figure 39: Dissociation curves for plate 1 (A) and plate 2 (B) for RAGE.

eNOS - The amplification curves, provided in Figure 40, showed that all samples had adequate PCR efficiency except for a single well associated with a BVM cultured with DHCAECs in elevated glucose media. The dissociation curves in Figure 41 revealed that all samples produced a single PCR product. 


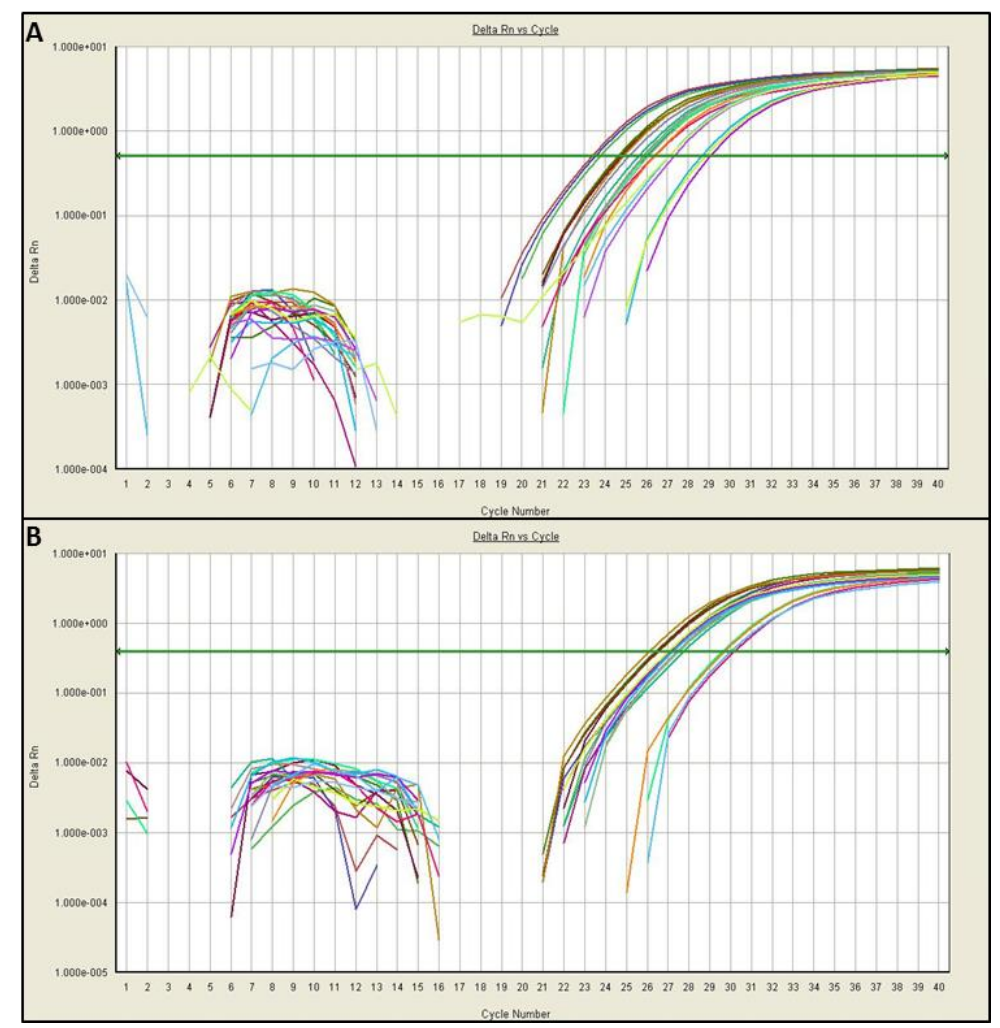

Figure 40: Amplification curves for plate 1 (A) and plate 2 (B) for eNOS.

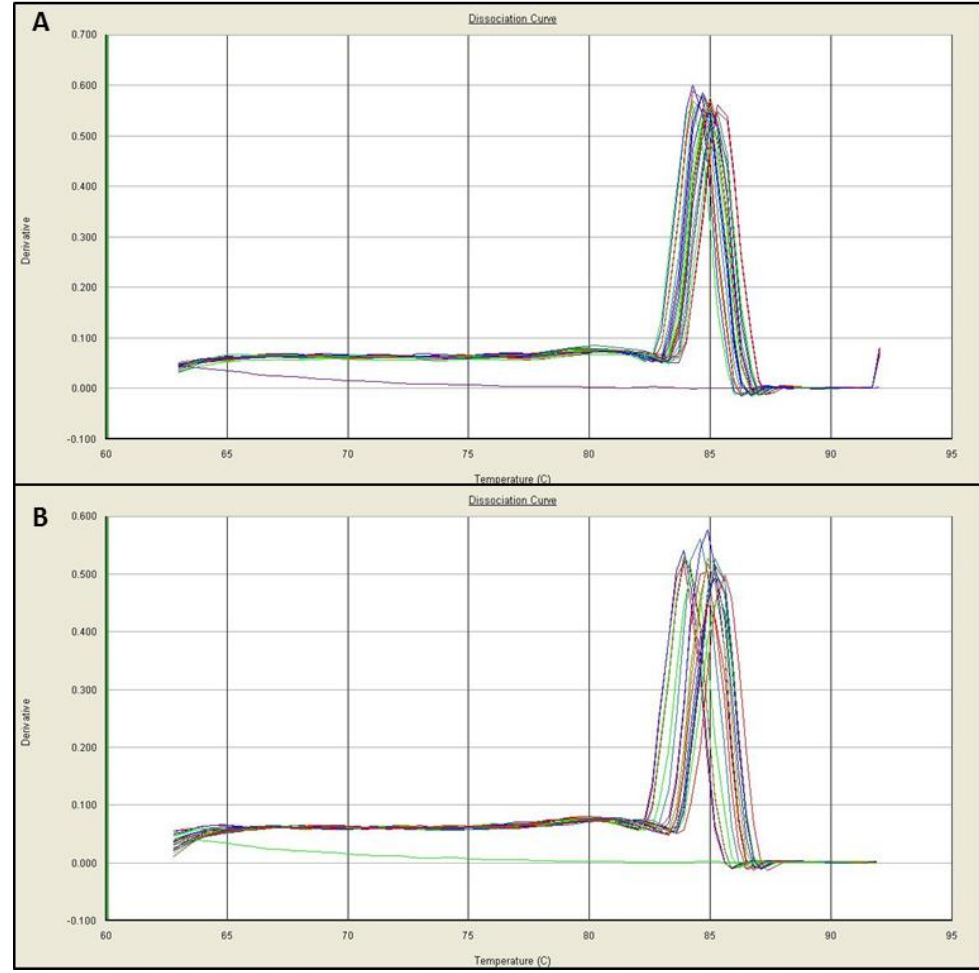

Figure 41: Dissociation curves for plate 1 (A) and plate 2 (B) for eNOS. 


\subsubsection{Quantitative Analysis}

PECAM - The statistical analysis of PECAM gene expression did not reveal any significant differences between the treatment groups, as seen in Figure 42; however there is a notable trend. PECAM expression was increased in DHCAECs compared to HUVECs regardless of glucose treatment. The glucose did not appear to have a large effect on HUVECs, but there was an increase in PECAM expression in DHCAECs cultivated in high glucose conditions compared to the control media.

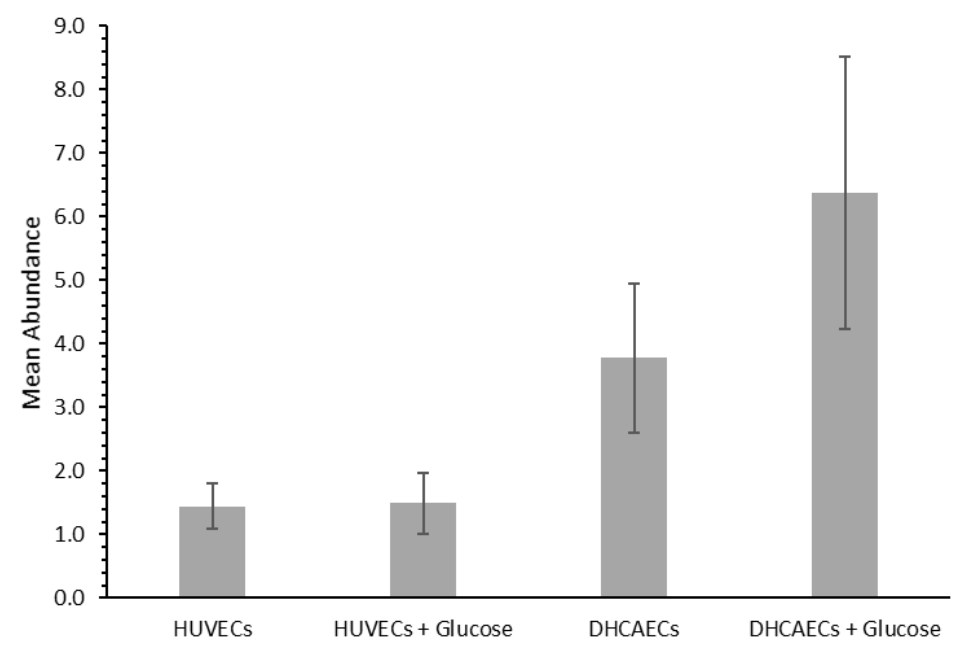

Figure 42: Relative abundance of PECAM for each treatment group.

$R A G E$ - The relative expression of $R A G E$ was significantly different between HUVECs that were cultured in control media compared to high glucose media as shown in Figure 43. There was also a significant difference in $R A G E$ expression between HUVECs and DHCAECs grown in control media, while RAGE expression in HUVECs and DHCAECs grown in high glucose media were relatively similar. Finally, there was also a significant difference between HUVECs in control media and DHCAECs in high glucose media. 


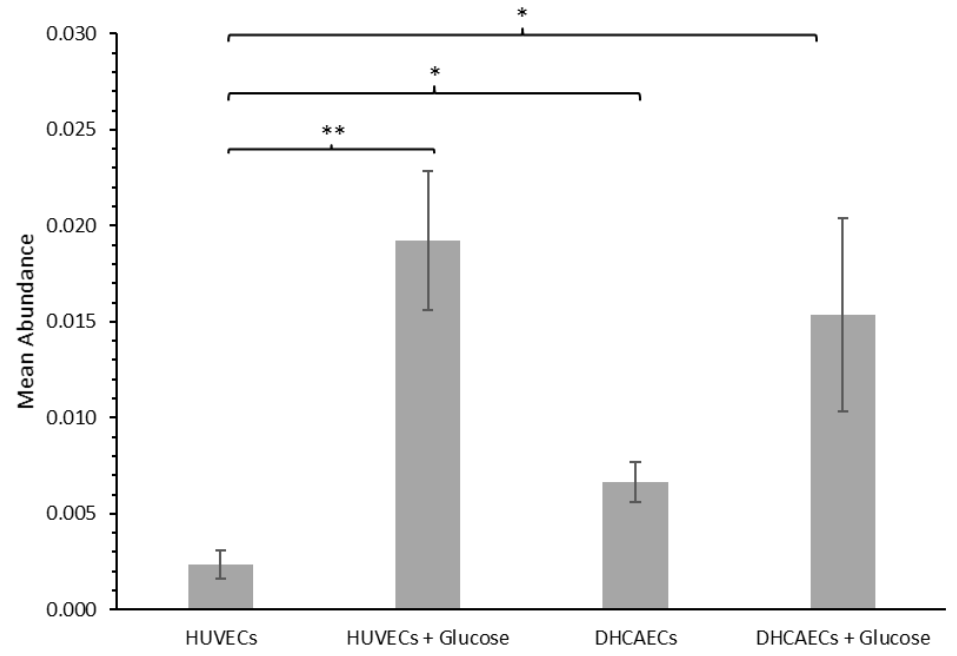

Figure 43: Relative abundance of $R A G E$ for each treatment group, where $* \mathrm{p}<0.05$ and $* * \mathrm{p}<0.01$

eNOS - eNOS expression was found to be significantly different between cell types cultured in either control media or high glucose media, as shown in Figure 44. Specifically, there was a significant increse in eNOS expression between HUVECs cultured in high glucose media and DHCAECs cultured in high glucose, as well as between media HUVECs cultured in control media and DHCAECs cultured in control media. There was also a significant difference in eNOS expression in DHCAECs cultured in control media versus high glucose media, with the high glucose condition showing lower $e N O S$ exporession. Finally, there was a significant difference between HUVECs in control media and DHCAECs in high glucose media, with the high glucose DHCAEC condition expressing higher $e N O S$. 


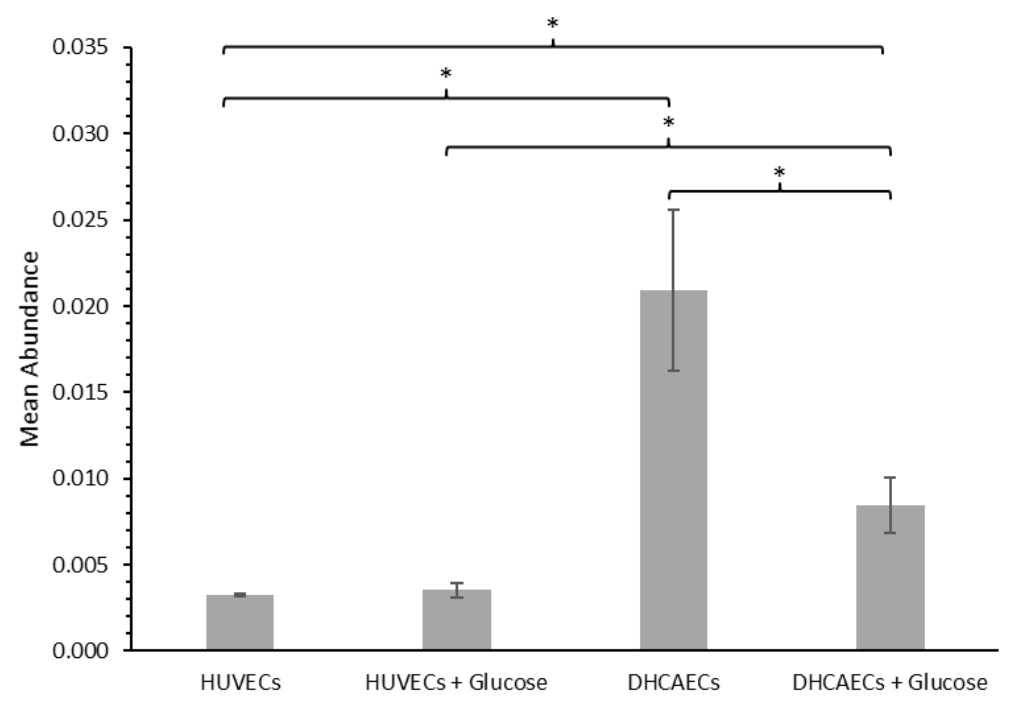

Figure 44: Relative abundance of $e N O S$ for each treatment group, where * $\mathrm{p}<0.05$.

\subsection{Discussion}

From the evaluation of the blood vessel mimics, it is evident that there are differences between using HUVECs and DHCAECs, as well as using media with added glucose to recreate the hyperglycemic condition diabetic blood vessels experience in vivo. From the immunofluorescence and SEM images, it was noted that cell deposition on and within the PLGA scaffolds did occur, although the cells were sparsely distributed with no areas of a cell "lining". The SEM images showed minimal cell deposition within the lumen of the scaffold, which may be due to poor handling of the specimens throughout the fixing and drying process. This could have been minimized by including more than one sample of BVM for each experimental group. Another possibility for poor cell coverage could have been not using enough cells for sodding each BVM, this could be mitigated by using more cells during the sodding of the BVMs. For the diabetic cells and the groups treated with high glucose media, there is the potential that disease processes associated diabetes 
and exposure of endothelial cells to elevated glucose concentrations could have affected cell functions, especially those associated with adhesion proteins [33, 41].

The immunofluorescence images show cell presence primarily in the lumen of the scaffold, which is consistent with previous analysis of BVMs produced in the Cal Poly Tissue Engineering lab. There was some migration of cells into the scaffold, but it was not substantial. During the course of taking the immunofluorescence images, it was noticed that the PLGA scaffold produces some background fluorescence when samples were observed under the widefield fluorescence microscope. An unrelated concurrent study being performed in the Tissue Engineering lab had similar results when imaging the same stains using confocal fluorescence microscopy. Unfortunately, a solution for this was not found during this study; therefore, the immunofluorescence images used in this study should be interpreted with the knowledge that the results may not accurately represent the amount of PECAM present. This supports the conclusion drawn in the preliminary study that immunofluorescence may not be as useful in studies that only utilize one cell type.

Scanning electron microscopy also revealed very sparse endothelial cell coverage in the BVMs for all experimental groups. It appeared that the cell coverage for the HUVECs and DHCAECs grown in control media were slightly more confluent than the cell coverage for the HUVECs and DHCAECs grown in high glucose media. This may be explained by an observation made in the preliminary study, that the cells grown in high glucose media tended to be less confluent in cell flasks when grown for the same period of time. This may also be indicative of apoptosis caused by ROS overproduction by endothelial cells exposed to elevated glucose conditions [57]. Although this is not 
ideal for endothelial confluency, it is consistent with endothelial cell responses to high glucose conditions in diabetes.

From the gene expression analysis of the BVMs, it appeared that cell type and media glucose concentration are both important factors to consider in developing a diabetic BVM model. Although there were no significant differences in PECAM expression, an upward trend was evident from HUVECs to DHCAECs, as well as from control media to high glucose media. This finding is consistent with the processes discussed in chapter 1 that found PECAM expression in HUVECs was not altered when cultured in high glucose conditions compared to normal glucose conditions [41].

For cells cultured in high glucose media, there was a significant increase in $R A G E$ expression compared to cells cultured in the control media. This is a promising finding for simulating diabetic processes in the BVM. The production of advanced glycation end products (AGEs) occurs in endothelial cells exposed to an excess of glucose, and when AGEs bind to their receptor $(R A G E)$, there is an increase in the production of reactive oxygen species (ROS) [43]. The increase in ROS causes oxidative stress within the vessels, propagating mechanisms responsible for the development of endothelial dysfunction in diabetics. Although both cell types exhibited this increase in $R A G E$ expression, there was also a significant increase in $R A G E$ expression between the two cell types in control media, which suggests that DHCAECs behave differently than HUVECs in the $3 \mathrm{D}$ culture condition.

The $e N O S$ expression data showed a significant increase in expression for DHCAECs cultured in control media compared to HUVECs in control media, as well as DHCAECs cultured in control media compared to DHCAECs cultured in high glucose 
media. Although it was expected that $e N O S$ expression would increase in DHCAECs compared to HUVECs, it was surprising to see increased $e N O S$ expression in the DHCAECs in control media rather than in high glucose media. There are several possible reasons for this: (1) introduction of endothelial cells to 3D dynamic culture conditions and (2) the lack of other cell types in the model. It has been shown that endothelial cells exposed to shear stress, such as flowing media, have increased activation of PECAM, which controls an alternative signaling pathway that increases eNOS activity [63]. By adding the cells into 3D cultivation in BVMs along with peristaltic flow of cell media, there may have been multiple signaling pathways initiated - since the presence of PECAM is likely due to the increased expression shown above.

Also, this model only incorporates endothelial cells, but in vivo nitric oxide (NO), produced by $e N O S$ activity, interacts largely with vascular smooth muscle cells(VSMCs), causing vasodilation [26]. It has been shown that vascular smooth muscle cells (VSMC) not only respond to NO but can also produce it [64]. Stimulation of VSMC induces NO release from these cells by stimulating the expression of an inducible nitric oxide synthase (iNOS) isoform, thus creating a negative feedback mechanism by decreasing the expression of eNOS [64]. This shows the importance of VSMC in the regulation of eNOS protein expression in endothelial cells and the involvement of VSMC in the regulation of inflammation-related endothelial dysfunction [64]. In diabetic patients, there are signaling pathways involving inflammation, endothelial dysfunction, and VSMCs - which are not included in this study, but are all important in creating an in vitro environment that accurately represents a diabetic blood vessel. 
Overall, this BVM study showed that 3D culturing conditions of HUVECs and DHCAECs differs from 2D culturing conditions, as shown by the gene expression results reflecting diabetic processes. This larger study also included more replicates for each experimental group, and allowed for better statistical analysis.

\subsection{Conclusion}

The aim of this study was to improve upon the previous diabetic blood vessel mimic produced in the Cal Poly Tissue Engineering Lab by including the addition of a high glucose environment. There were also protocol changes that were implemented based on the findings in the preliminary study, specifically using a pre-made sterilized glucose solution to add to the cell media to create an elevated glucose environment, eliminating the middle range glucose concentration group, and incorporating the $3 \mathrm{D}$ culturing condition by completing a BVM set-up with the experimental groups. The BVMs were then evaluated using immunofluorescence, SEM and qPCR gene expression. The immunofluorescence and SEM analysis suggested that the implementation of high glucose cell media is feasible for endothelial cell deposition in the BVM. Gene expression data was consistent with previous studies for PECAM expression in both the DHCAEC and HUVEC groups, and both the high glucose and normal glucose groups, which confirms the ability of qPCR analysis to be applied to the BVM set up. The gene expression results for $R A G E$ and $e N O S$ are mostly consistent with prior studies that show differences in expression for high glucose conditions compared to normal glucose conditions, as well as for diabetic cells compared to healthy cells $[45,65,66]$. 
This is a promising foundation for applying gene expression analysis in BVM studies to determine if the pathophysiologic processes of endothelial dysfunction associated with diabetes are occurring in vitro. 


\section{Chapter 5: Discussion and Conclusion}

\subsection{Summary and Aims of the Thesis}

Diabetes and coronary artery disease are highly correlated $[4,7,8]$. With the hyperglycemia that accompanies diabetes, there are multiple mechanisms that are activated that contribute to the progression of vascular disease, namely endothelial dysfunction. The devices used to treat vascular disease have not been well-tested for efficacy in diabetic patients. Although preclinical diabetic models exist, such as streptozotocin-induced diabetic rats and mice, spontaneously mutated rats such as Zucker Diabetic Fatty Rats (ZDF), genetically modified mice models, diabetic Yucatan miniswine, and streptozotocin-induced diabetic domestic pigs, there are limitations to these models, and the medical device industry would benefit from earlier stage diabetic testing environments $[19,20,21]$. In the Cal Poly Tissue Engineering lab, blood vessel mimics are created to test intravascular devices in vitro. In order to facilitate testing of intravascular devices intended for use in diabetic patients, a diabetic BVM was previously created using diabetic human coronary artery endothelial cells (DHCAECs) and diabetic human coronary artery smooth muscle cells (DHCSMCs) [30, 67]. Although this model demonstrated the ability to apply diseased cell types to the BVM set up, there was a lack of analysis to demonstrate if the diabetic BVM accurately modeled diabetic disease processes.

The first aim of this thesis - to establish a protocol for qPCR analysis of BVMs cultivated with HUVECs and DHCAECs- was accomplished by performing a preliminary study of primer verification for qPCR. From this preliminary study, it was found that the selected genes of interest and the reference gene chosen had at least one 
accompanying DNA primer that produced efficient PCR amplification and a single, desired PCR product. From these findings, the optimal DNA primer - of the primers tested - was selected for each gene of interest and implemented in the subsequent studies. This study also established that PECAM, RAGE, and $e N O S$ are suitable genes of interest - due to their involvement in the pathophysiology of endothelial dysfunction in relation to diabetes and hyperglycemia - and were expressed in both HUVECs and DHCAECs, cultured in either normal glucose concentration media or high glucose concentration media. GAPDH was also determined to be an appropriate reference gene for qPCR data analysis because it was unaffected by differences in cell type or experimental treatment.

The second aim of this thesis was to improve upon the diabetic BVM by developing and implementing an in vitro hyperglycemic condition through the addition of glucose to cell culture media. This was investigated in a preliminary study using a normal glucose concentration, a mildly elevated glucose concentration of $10.5 \mathrm{mM}$, and a high glucose concentration of $25.5 \mathrm{mM}$ in cell media to culture HUVECs and DHCAECs in static culture conditions in cell flasks. From this study, it was determined that a concentration of $25.5 \mathrm{mM}$ of glucose elicits a measurable change in gene expression from endothelial cells without causing widespread apoptosis. This glucose concentration was then used to produce a "diabetic" environment for culturing BVMs.

Using the glucose concentration of $25.5 \mathrm{mM}$ established in the preliminary study, BVMs were cultured with either HUVECs, or DHCAECs and in either control, or high glucose cell media. Two, three, or four biological replicates - individual BVMs - were produced for each treatment group to increase the sample size for qPCR analysis. Through immunofluorescence and SEM imaging analysis, it was shown that all treatment 
groups resulted in at least a small amount of endothelial cell deposition throughout the lumen of the BVM. This demonstrated the practicality of culturing a BVM with DHCAECs in a hyperglycemic model.

From gene expression analysis, it was found that there were no significant differences in PECAM abundance in mRNA of DHCAECs compared to HUVECs, as well as in the groups cultured in a high glucose environment compared to those grown in control media. This was consistent with a previous study that showed that HUVECs cultured in high glucose conditions $(30.0 \mathrm{mM})$ in vitro did not have altered PECAM expression compared to HUVECs cultured in normal $(5.0 \mathrm{mM})$ glucose conditions [41]. Although there are limited studies on direct comparisons of diabetic and healthy human endothelial cells in vitro - especially regarding gene expression of PECAM - it is suggested that, due to higher incidence of atherosclerosis in diabetic patients, high glucose and insulin levels can promote increased inflammation in cultured endothelial cells in a PECAM-dependent matter [68]. This relationship between PECAM and inflammation in diabetic patients may explain the trend of increased PECAM abundance in the DHCAEC BVMs compared to the HUVEC BVMs. To obtain a better understanding of PECAM gene expression in BVMs, gene expression analysis should continue to be performed in future BVM studies.

There was a significant increase in $R A G E$ abundance in mRNA of DHCAECs cultured in control media compared to HUVECs cultured in control media, as well as in HUVECs treated with high glucose media compared to normal glucose media. These results are consistent with research that has shown that $R A G E$ expression increases in human aortic endothelial cells incubated in high glucose conditions compared to normal 
glucose conditions due to hyperglycemia-induced reactive oxygen species (ROS) production by the mitochondrial electron transport chain binding to NFKB to the $R A G E$ promoter [65]. Additionally, a previous study showed that $R A G E$ expression was increased in aortic endothelial cells of streptozotocin-induced diabetic mice as compared to non-diabetic mice, which is again consistent with the findings in this study of DHCAECs compared to HUVECs [65]. The expression of RAGE in DHCAECs as compared to HUVECs, and in cells cultured in high glucose culture conditions compared to normal glucose culture conditions was consistent with previous studies, indicating that this diabetic model has promise in accurately mimicking diabetic and hyperglycemic conditions in vivo. This also shows that both the diabetic condition and hyperglycemia are important in recreating pathophysiologic processes in vitro.

eNOS abundance in mRNA of DHCAECs in control media was significantly higher than all other treatment groups; DHCAECs of both glucose treatments were significantly higher than the corresponding HUVEC glucose treatment groups. There are conflicting studies showing both increased and decreased eNOS gene expression for human aortic endothelial cells cultured in high glucose conditions $(22.2 \mathrm{mM}-25 \mathrm{mM}$ concentrations) for a prolonged time (5-7 days) [45, 66]. Furthermore, it has been shown that mouse aortic endothelial cells from a diabetic population had reduced $e N O S$ gene expression compared to a control population [66]. The differences in $e N O S$ expression in prior studies and this study reveals the complex nature of the factors that affect $e N O S$ expression in endothelial dysfunction due to diabetes; however, this highlights the importance of including a high glucose culturing condition and a diabetic cell type to produce a representative physiologic environment in vitro for diabetic vascular 
conditions. To improve upon the diabetic BVM produced in this study, future studies should include VSMCs due to the important role of VSMCs in the exchange of nitric oxide with ECs that affects the expression of eNOS [64].

The gene expression findings, along with the observations from the immunofluorescence and SEM images, shows that the diabetic BVMs cultured in high glucose conditions are significantly different than the "healthy" BVMs that have been previously developed in the tissue engineering lab. There appears to be several characteristics of these diabetic BVMs cultured with a simulated hyperglycemic condition that indicate that disease processes of diabetic endothelial dysfunction are occurring in these models.

\subsection{Challenges and Limitations}

As previously discussed, the tissue engineered blood vessel mimic construct adequately models some, but not all aspects of blood vessels in vivo. In this study, there are several major features of biologic blood vessels that are lacking in BVMs that may affect the disease process of diabetes, one of which is the lack of cell types other than endothelial cells in the BVM. For instance, nitric oxide (NO) interacts with vascular smooth muscle cells and results in the alteration of signal transduction pathways involved in the progression of endothelial dysfunction - and most likely eNOS mRNA abundance [69]. Additionally, there were no immune cells present; therefore, the inflammation pathway is not activated in its entirety. Due to the involvement of PECAM with the inflammatory response, the lack of these cells may have affected the abundance of PECAM expression in mRNA in both endothelial cell types [68]. 
It should also be noted that glucose concentration was not actively monitored throughout the BVM set up. Although the cell media was changed frequently during the expansion of HUVECs and DHCAECs, once the BVMs were placed in the bioreactors and media was pumped through the BVM constructs there was no monitoring of glucose concentration. In these set-ups, cells actively consume glucose resulting in the decrease of glucose concentration over time, which may have altered the elevated glucose environment that was desired. Continuous monitoring and the addition of glucose solution to the BVM as necessary would improve the model developed in this study. Patients with diabetes have fluctuations in blood glucose levels throughout the day, therefore the continuous addition of glucose to maintain a steady glucose concentration is not necessary, but the addition of glucose after initial sodding of the cells could reflect these changes in blood glucose concentration seen in vivo.

Furthermore, the power of the statistical analysis was limited by the sample size of each study. The first study was limited to triplicate of each sample due to the lack of biological replicates; however, the final study that included biological replicates as well as technical triplicates was still limited to fewer data points due to the removal of samples for poor RNA quality at the time of isolation, as well as removal of qPCR samples if primer-dimer formation, inappropriate amplification or multiple qPCR products were observed on the amplification or dissociation curves. To increase the number of data points in future studies, more samples could be used for each experimental group. For those samples with poor RNA quality or did not meet the quality control standards for qPCR, this most likely due to improper handling of the samples and contamination, 
which can be mitigated through proper aseptic technique during preparation and storage of the samples.

Another limitation to this study was the sparse endothelial cell coverage in the lumen of the BVMs for all experimental groups elucidated by the scanning electron microscopy images. The lack of cells seen in the lumen of the BVMs may have affected gene expression data due to the scarcity of cells, which could cause RNA quality to be poor due to low concentrations during isolation. There are several possibilities as to why there were few cells in the lumen of the BVM: apoptosis caused by ROS overproduction by endothelial cells exposed to elevated glucose conditions, adhesion proteins altered by glucose conditions, or not enough cells used during seeding of the BVM $[57,70,71]$. Although this is not ideal for endothelial confluency, it is consistent with endothelial cell responses to high glucose conditions in diabetes and could be addressed in future studies by increasing the number of cells used for seeding of the BVM or including VSMCs seeded prior to endothelial cells due to the favorable cell-cell adhesion interactions over cell-scaffold interactions.

\subsection{Future Work}

From the limitations discussed, the diabetic BVM model can certainly be improved. The incorporation of smooth muscle cells has already been demonstrated in BVMs created in the tissue engineering lab for the healthy cell type; however, the addition of diabetic vascular smooth muscle cells in addition to the high glucose culturing condition would be a reasonable next step in the development of a more accurate diabetic BVM. 
As previously mentioned, the qPCR analysis should be repeated on future BVMs using the same genes of interest as this thesis because of the effects the interaction of smooth muscle cells and endothelial cells have on the gene expression of PECAM, RAGE, and eNOS. This also maintains consistency and allows the Cal Poly tissue engineering laboratory to accumulate more data on the BVMs being produced.

For the qPCR analysis of these cells, the number of genes selected for investigation was limited due to the size of the plates used for qPCR; however, moving forward with gene expression analysis, there are additional genes that could lead to a better understanding of the disease processes involved in diabetes and endothelial cells. One gene in particular is vascular cell adhesion molecule (VCAM), which plays a regulatory role in anchoring monocytes involved in the inflammatory reaction and contributes to the development atherosclerosis, particularly in its early phases $[8,40]$. This gene has been previously studied and shown to have increased expression in HUVECs in hyperglycemic conditions, as well as in alloxan-treated diabetic rabbits [40, 71]. These elevations are due to AGE-RAGE interactions in the development of endothelial dysfunction and as a consequence of cellular oxidant stress and the activation of transcription factor NFאB [40, 71].

Due to the lack of information garnered from the immunofluorescence images, it is suggested that live-dead immunofluorescence staining be performed in the BVMs immediately after take down, before fixing cells. This would allow for the observation of apoptotic or necrotic activity that may be associated with the diabetic condition. Alternatively, a gene that is associated with apoptotic activity, such as anti-apoptotic protein B-cell lymphoma 2 (Bcl-2) and pro-apoptotic protein Bcl-2-associated X protein 
(Bax), could be included in qPCR analysis [70]. These genes would be extremely useful for diabetic purposes specifically because it has been shown that there is a significant decrease of Bcl-2 and a simultaneous increase in Bax expression when HUVECs were cultured in a stable high glucose environment [70].

Finally, in addition to glucose, insulin should be investigated as a supplement to the cell media. For type 2 diabetes, the inability of cells to utilize insulin leads to the hyperglycemic condition. This causes an increase in insulin levels in the blood - or hyperinsulinemia. Along with hyperglycemia, hyperinsulinemia is known to cause endothelial dysfunction by increasing oxidant stress through increased endothelin-1, which induces $\mathrm{NAD}(\mathrm{P}) \mathrm{H}$ oxidase expression and increased generation of superoxide anions in human endothelial cells [72]. Insulin is also known to regulate the expression of eNOS gene which alters vascular tone, which may lead to endothelial dysfunction in diabetics [73]. Thus, it is suggested that adding insulin to replicate modest hyperinsulinemia, mimicking fasting hyperinsulinemia of insulin-resistant states - such as diabetes mellitus - will better represent the diabetic condition of blood vessels in vitro with respect to the pathophysiology of endothelial dysfunction [72].

\subsection{Conclusion}

Overall, it was found that qPCR analysis was feasible and applicable to tissue engineered blood vessels cultured with HUVECs and DHCAECs. This analysis method allows for more in-depth characterization of these BVMs, especially in distinguishing differences between the two cell types. 
It was also found that a high glucose environment - in addition to incorporating diabetic endothelial cells - in the production of BVMs leads to a BVM that has significantly different characteristics than the standard "healthy" BVMs previously created in the Cal Poly Tissue Engineering Lab. This was confirmed with the use of immunofluorescence, SEM imaging, and qPCR analysis. The qPCR analysis of these BVMs was somewhat consistent with the proposed hypotheses: PECAM, RAGE, and eNOS expression were increased in DHCAECs cultured in a high glucose environment, compared to the HUVECs cultured in a normal glucose environment. It was found that $R A G E$ and $e N O S$ expression was significantly increased between these treatment groups; however, PECAM expression was not significantly increased, but had an upward trend.

From these studies, it is suggested that high glucose media should be implemented in the production of diabetic BVMs with DHCAECs, as well as including vascular smooth muscle cells, for all future studies of the diabetic condition in conjunction with tissue engineered blood vessel mimics. qPCR gene expression analysis should also be implemented for all future studies of BVMs, as it allows for further characterization of the BVMs, and a quantitative analysis of cell function. 


\section{REFERENCES}

[1] Centers for Disease Control and Prevention, "National Diabetes Statistics Report: Estimates of Diabetes and its Burden in the United States, 2014," US Department of Health and Human Services, Atlanta, 2014.

[2] J. E. Shaw, R. A. Sicree and P. Z. Zimmet, "Global estimates of the prevalence of diabetes for 2010 and 2030," Diabetes Res Clin Pract, vol. 87, no. 1, pp. 4$14,2010$.

[3] American Diabetes Association, "Diagnosis and Classification of Diabetes Mellitus," Diabetes Care, vol. 27, no. suppl 1, pp. s5-s10, 2004.

[4] J. Forbes and M. Cooper, "Mechanisms of diabetic complications," Physiol Rev, vol. 93, no. 1, pp. 137-88, Jan 2013.

[5] A. Nouwen, G. Nefs, I. Caramlau, M. Connock, K. Winkley, C. E. Lloyd, M. Peyrot and F. Pouwer, "Prevalence of Depression in Individuals with Impaired Glucose Metabolism or Undiagnosed Diabetes: A systematic review and meta-analysis of the European Depression in Diabetes (EDID) Research Consortium," Diabetes Care, vol. 34, no. 3, pp. 752-762, March 2011.

[6] T. Cukierman, H. C. Gerstein and J. D. Williamson, "Cognitive decline and dementia in diabetes - systematic overview of prospective observational studies," Diabetologia, vol. 48, pp. 2460-2469, 2005.

[7] S. M. Haffner, S. Lehto, T. Ronnemaa, K. Pyorala and M. Laakso, "Mortality from Coronary Heart Disease in Subjects with Type 2 Diabetes and in Nondiabetic Subjects with and without Prior Myocardial Infarction," N Engl J Med, vol. 
339, pp. 229-234, 1998.

[8] H. A. Hadi and J. A. Suwaidi, "Endothelial dysfunction in diabetes mellitus," Vasc Health Risk Manag, vol. 3, no. 6, pp. 853-876, Dec 2007.

[9] F. Giacco and M. Brownlee, "Oxidative stress and diabetic complications," Circ Res, vol. 107, no. 9, pp. 1058-1070, Oct 2010.

[10] S. S. Chung, E. C. Ho, K. S. Lam and S. K. Chung, "Contribution of polyol pathway to diabetes-induced oxidative stress," J Am Soc Nephrol, vol. 14, no. 8 Suppl 3, pp. S233-S236, Aug 2003.

[11] H. Lodish, A. Berk, S. Zipursky and e. al., "Section 16.1 Oxidation of Glucose and Fatty Acids to CO2," in Molecular Cell Biology. , 4th ed., New York, W. H. Freeman, 2000.

[12] A. Goldin, J. A. Beckman, A. M. Schmidt and M. A. Creager, "Basic Science for Clinicians: Advanced Glycation End Products - Sparking the Development of Diabetic Vascular Injury," Circulation, vol. 114, pp. 597-605, 7 Aug 2006.

[13] K. Hess, N. Marx and M. Lehrke, "Cardiovascular disease and diabetes: the vulnerable patient," Eur Heart J Suppl, vol. 14, no. Suppl B, pp. B4-B13, 2012.

[14] D. M. Nathan, P. A. Cleary, C. Jye-Yu, M. S. Backlund, M. S. Genuth, J. M. Lachin, T. J. Orchard, P. Raskin and B. Zinman, "Intensive Diabetes Treatment and Cardiovascular Disease in Patients with Type 1 Diabetes," N Engl J Med, vol. 353 , no. 25 , pp. 2643-2653, Dec 2005.

[15] K. Uchiyama, H. Ino, K. Hayashi, K. Fujioka, S. Takabatake, J. Yokawa, M. 
Namura and e. al., "Impact of Severe Coronary Disease Associated or Not Associated with Diabetes Mellitus on Outcome of Interventional Treatment Using Stents: Results from HERZ (Heart Research Group of Kanazawa) Analyses," Journal of International Medical Research, vol. 39, pp. 549-557, 2011.

[16] R. Corpus, "Treatment of CAD in patients with diabetes - what do we really know?," Diab Vasc Dis Res, vol. 1, no. 1, p. 33, May 2004.

[17] V. A. Fonseca, "Management of Diabetes Mellitus and Insulin Resistance in Patients with Cardiovascular Disease," Am J Cariol, vol. 92, no. Suppl, pp. 50J-60J, 2003.

[18] G. De Luca, R. Sauro, M. Capasso, T. Lanzillo, F. Manganelli, G. Carbone, F. Lanni, M. R. Pagliuca, V. Palmieri, V. Serino, G. Rosato, H. Suryapranata and E. Di Lorenzo, "Impact of Diabetes on the Benefits from EverolimusEluting Stent as Compared to First-Generation Drug-Eluting Stent in Patients with ST Elevation Myocardial Infarction," Diab Vasc Dis Res, vol. 12, no. 5, pp. 306-314, Jul 2015.

[19] H. Song, P. W. Zandstra and M. Radisic, "Engineered heart tissue model of diabetic myocardium," Tissue Eng Part A, vol. 17, no. 13-14, pp. 1869-78, Jul 2011.

[20] C. Von Wilmowsky, P. Stockmann, P. Metzler, I. A. Harsch, K. Amann and K. A. Schlegel, "Establishment of a streptozotocin-induced diabetic domestic pig model and a systematic evaluation of pathological changes in the hard and soft tissue over a 12-month period," Clin Oral Implants Res, vol. 21, no. 7, 
pp. 709-717, Jul 2010.

[21] H. B. van der Worp, D. W. Howells, E. S. Sena, M. J. Porritt, S. Rewell, V. O'Collins and M. R. Macleod, "Can Animal Models of Disease Reliably Inform Human Studies?," PLoS Med, vol. 7, no. 3, p. e1000245, 2010.

[22] R. Lanza, R. Langer and J. Vacanti, Principles of Tissue Engineering, 3rd ed., Burlington, MA: Elsevier, 2007.

[23] N. L'Heureux, N. Dusserre, A. Marini, S. Garrido, L. de la Fuente and T. McAllister, "Techonolgy Insight: The Evolution of tissue-engineered vascular grafts-from research to clinical practice," Nat Clin Pract Cardiovasc Med, vol. 4, no. 7, pp. 389-95, Jul 2007.

[24] K. O. Cardinal, G. T. Bonnema, H. Hofer, J. K. Barton and S. K. Williams, "TissueEngineered Vascular Grafts as In Vitro Blood Vessel Mimics for the Evaluation of Endothelialization of Intravascular Devices," Tissue Eng, vol. 12, no. 12, pp. 3431-8, Dec 2006.

[25] S. Herting, A. DiBartolomeo, T. Pipes, S. Kunz, K. Temnyk, J. Truty, S. Ur and K. O. Cardinal, "Human Umbilical Versus Coronary Cell Sources for TissueEngineered Blood Vessel Mimics," Applied In Vitro Toxicology, vol. 2, no. 3, pp. 175-182, Sept 2016.

[26] E. N. Marieb and K. N. Hoehn, Human Anatomy \& Physiology, 9th ed., Glenview, IL: Pearson Education Inc., 2009.

[27] A. Hasan, A. Memic, N. Annabi, M. Hossain, A. Paul, M. R. Dokmeci, F. Dehghani and A. Khademhosseini, "Electrospun scaffolds for tissue engineering of 
vascular grafts," Acta Biomaterialia, vol. 10, no. 1, pp. 11-25, 2013.

[28] T. R. Pena, "Preparation and Characterization of Electrospun Poly(D,L-lactide-coglycolide) Scaffolds for Vascular Tissue Engineering and the Advancement of an In Vitro Blood Vessel Mimic," Digital Commons @ Cal Poly, San Luis Obispo, 2009.

[29] S. Ur, "Characterization of Blood Vessel Mimics by Gene Expression and the Implementation of Novel Cell Types," pp. 1-47, 23 Sept 2013.

[30] V. Mediratta, "Implementation and Assessment of Hyperglycemic Conditions for the Creation of a Diabetic Blood Vessel Mimic," Digital Commons @ Cal Poly, San Luis Obispo, 2011.

[31] Sigma Aldrich, "Glucose in Cell Culture," [Online]. Available: http://www.sigmaaldrich.com/life-science/cell-culture/learning-center/mediaexpert/glucose.html\#Primary_Functions. [Accessed September 2015].

[32] J.-M. Fritschy and W. Hartig, "Immunofluorescence," eLS, Apr 2001.

[33] H. M. DeLisser, H. S. Baldwin and S. M. Albelda, "Platelet Endothelial Cell Adhesion Molecule 1 (PECAM-1/CD31): A Multifunctional Vascular Cell Adhesion Molecule," Trends in Cardiovascular Medicine, vol. 7, no. 6, pp. 203-210, Aug 1997.

[34] D. E. Jackson, "The unfolding tale of PECAM-1," FEBS Letters, vol. 540, no. 1-3, pp. 7-14, Mar 2003.

[35] A. C. Jimenez-Vergara, V. Guiza-Arguello, S. Becerra-Bayona, D. J. Munoz-Pinto, R. E. McMahon, A. Morales, L. Cubero-Ponce and M. S. Hahn, "Approach 
for Fabricating Tissue Engineered Vascular Grafts with Stabel Endothelialization," Annals of Biomedical Engineering, vol. 38, no. 9, pp. 2885-2895, Sept 2010.

[36] W. He, Z. Ma, W. E. Teo, Y. X. Dong, P. A. Robless, T. C. Lim and S. Tamakrishna, "Tubular nanofiber scaffolds for tissue engineered smalldiameter vascular grafts," Jounal of Biomedical Materials Research Part A, vol. 90A, no. 1, pp. 205-216, Jun 2009.

[37] S. H. Ku and C. B. Park, "Human endothelial cell growth on mussel-inspired nanofiber scaffod for vascular tissue engineering," Biomaterials, vol. 31, no. 36, pp. 9431-9437, Dec 2010.

[38] F. R. Pu, R. L. Williams, T. K. Markkula and J. A. Hunt, "Expression of leukocyteendothelial cell adhesion molecules on monocyte adhesion to human endothelial cells on plasma treated PET and PTFE in vitro," Biomaterials, vol. 23, no. 24, pp. 4705-4718, Dec 2002.

[39] H. D. VanGuilder, K. E. Vrana and W. M. Freeman, "Twenty-five years of quantitative PCR for gene expression analysis," BioTechniques, vol. 44, no. 5, pp. 619-626, Apr 2008.

[40] D. Onat, D. Brillon, P. C. Colombo and A. M. Schmidt, "Human Vascular Endothelial Cells: A Model System for Studying Vascular Inflammation in Diabetes and Atherosclerosis," Curr Diab Rep, vol. 11, no. 3, pp. 193-202, Jun 2011.

[41] S. M. Baumgartner-Parzer, L. Wagner, M. Pettermann, A. Gessl and W. Waldhausl, 
"Modulation by high glucose of adhesion molecule expression in cultured endothelial cells," Diabetologia, vol. 38, no. 11, pp. 1367-70, Nov 1995.

[42] L. Feng, C. Matsumoto, A. Schwartz, A. M. Schmidt, D. M. Stern and J. PileSpellman, "Chronic Vascular Inflammation in Patients with Type 2 Diabetes," Diabetes Care, vol. 28, no. 2, pp. 379-384, Feb 2005.

[43] M.-P. Wautier, O. Chappey, S. Corda, D. M. Stern, A. M. Schmidt and J.-L. Wautier, "Activation of NADPH oxidase by AGE links oxidant stress to altered gene expression via RAGE," Am J Physiol Endocrinol Metab, vol. 280, no. 5, pp. E685-E694, May 2001.

[44] U. Hink, H. Li, H. Mollnau, M. Oelze, M. Hartmann, M. Skatchkov, F. Thaiss, R. A. Stahl, A. Warnholtz, T. Meinertz, K. Griendling, D. G. Harrison, U. Forstermann and T. Munzel, "Mechanisms Underlying Endothelial Dysfunction in Diabetes Mellitus," Circulartion Research: Journal of the American Heart Association, vol. 88, no. 2, pp. e14-e22, Feb 2001.

[45] F. Cosentino, K. Hishikawa, Z. S. Katusic and T. F. Luscher, "High Glucose Increases Nitric Oxide Synthase Expression and Superoxide Anion Generation in Human Aortic Endothelial Cells," Circulation, vol. 96, pp. 2528, Jul 1997.

[46] National Center for Biotechnology Information, "Polymerase Chain Reaction (PCR)," U.S. National Library of Medicine, 26 September 2014. [Online]. Available: https://www.ncbi.nlm.nih.gov/probe/docs/techpcr/. [Accessed January 2016]. 
[47] J. Bartlett and D. Stirling, "A Short History of the Polymerase Chain Reaction," PCR Protocols, vol. 226, no. 2, pp. 3-6, 2003.

[48] R. S. Matson, Microarray Methods and Protocols, 1st ed., Boca Raton, FL: CRC Press, 2009, p. 216.

[49] S. Taylor, M. Wakem, G. Dijkman, M. Alsarraj and M. Nguyen, "A practical approach to RT-qPCR--Publishing data that conform to the MIQE guidelines," Methods, vol. 50, no. 4, pp. S1-S5, 2010.

[50] B. Zyzynska-Granica and K. Koziak, "Identification of Suitable Reference Genes for Real-Time PCR Analysis of Statin-Treated Human Umbilical Vein Endothelial Cells," PLoS ONE, vol. 7, no. 12, p. e51547, Dec 2012.

[51] B. Kozera and M. Rapacz, "Reference genes in real-time PCR," Journal of Applied Genetics, vol. 54, no. 4, pp. 391-406, Nov 2013.

[52] O. Thellin, W. Zorzi, B. Lakaye, B. De Borman, B. Coumans, G. Hennen, T. Grisar, A. Igout and E. Heinen, "Housekeeping genes as internal standards: use and limits," J Biotechnol, vol. 75, no. 2-3, pp. 291-5, Oct 1999.

[53] M. W. Pfaffl, A. Tichopad, C. Prgomet and T. P. Neuvians, "Determination of stable housekeeping genes, differentially regulated target genes and sample integrity: BestKeeper - Excel-based tool using pair-wise correlations," Biotechnology Letters, vol. 26, no. 6, pp. 509-515, Mar 2004.

[54] S. Bakhashab, S. Lary, F. Amed, H.-J. Schulten, A. Bashir, F. W. Ahmed, A. L. AlMalki, H. S. Jamal, M. A. Gari and J. U. Weaver, "Reference Genes for Expression Studies in Hypoxia and Hyperglycemia Models in Human 
Umbilical Vein Endothelial Cells," G3: Genes, Genomes, Genetics, vol. 4, no. 11, pp. 2159-2165, Nov 2014.

[55] B. Thornton and C. Basu, "Real-time PCR (qPCR) primer design using free online software," Biochemistry and Molecular Biology Education, vol. 39, no. 2, pp. 145-154, March 2011.

[56] K. M. Ririe, R. P. Rasmussen and C. T. Wittwer, "Product Differentiation by Analysis of DNA Melting Curves during the Polymerase Chain Reaction," Analytical Biochemistry, vol. 245, no. 2, pp. 154-160, Feb 1997.

[57] L. Piconi, L. Quagliaro, R. Assaloni, R. Da Ros, A. Maier, G. Zuodar and A. Ceriello, "Constant and intermittent high glucose enhances endothelial cell apoptosis through mitochondrial superoxide overproduction," Diabetes Metabolism Research and Reviews, vol. 22, no. 3, pp. 198-203, Feb 2006.

[58] G. Viberti, "A Glycemic Threshold for Diabetic Complications?," New England Journal of Medicine, vol. 332, pp. 1293-1294, May 1995.

[59] J. H. Fuller, M. J. Shipley, G. Rose, R. J. Jarrett and H. Keen, "Mortality from coronary heart disease and stroke in relation to degree of glycaemia: the Whitehall study," Br Med J (Clin Res Ed), vol. 287, pp. 867-870, Sept 1983.

[60] R. Edmonson, J. J. Broglie, A. F. Adcock and L. Yang, "Three-Dimensional Cell Culture Systems and Their Applications in Drug Discovery and Cell-Based Biosensors," Assay Drug Dev Technol, vol. 12, no. 4, pp. 207-218, May 2014.

[61] K. O. Cardinal and S. K. Williams, "Assessment of Intimal Response to a Protein- 
Modified Stent in a Tissue-Engineered Blood Vessel Mimic," Tissue

Engineering: Part A, vol. 15, no. 12, pp. 3869-3876, 2009.

[62] M. Gibbons and S. Ur, "Development and Characterization of PLGA and ePTFE Blood Vessel Mimics Using Gene Expression Analysis," Digital Commons @ Cal Poly, San Luis Obispo, 2012.

[63] I. Fleming, B. Fisslthaler, M. Dixit and R. Busse, "Role of PECAM-1 in the shearstress-induced activation of Akt and the endothelial nitric oxide synthase (eNOS) in endothelial cells," Journal of Cell Science, vol. 118, pp. 4103$4111,2005$.

[64] T. De Frutos, L. S. De Miguel, M. Garcia-Duran, F. Gonzalez-Fernandez, J. A. Rodriguez-Feo, M. Monton, J. Guerra, J. Farre, S. Casado and A. LopezFarre, "NO from smooth muscle cells decreases NOS expression in endothelial cells: role of TNF-a," American Journal of Physiology-Heart and Circulatory Physiology, vol. 277, no. 4 Part 2, pp. H1317-1325, Nov 1999.

[65] D. Yao and M. Brownlee, "Hyperglycemia-Induced Reactive Oxygen Species Increase Expression of the Receptor for Advanced Glycation End Products (RAGE) and RAGE Ligands," Diabetes, vol. 59, no. 1, pp. 249-255, Jan 2010.

[66] S. Srinivasan, M. E. Hatley, D. T. Bolick, L. A. Palmer, D. Edelstein, M. Brownlee and C. C. Hedrick, "Hyperglycaemia-induced superoxide production decreases eNOS expression via AP-1 activation in aortic endothelial cells," Diabetologia, vol. 47, no. 10, pp. 1727-1734, Oct 2004. 
[67] K. Temnyk, S. Kunz and K. O. Cardinal, "Development of a Tissue Engineered "Diabetic" Blood Vessel Mimic for Intravascular Device Testing," Cal Poly, San Luis Obispo, 2016.

[68] A. Woodfin, M.-B. Voisin and S. Nourshargh, "PECAM-1: A Multi-functional Molecule in Inflammation and Vascular Biology," Arteriosclerosis, Thrombosis, and Vascular Biology, vol. 27, no. 12, pp. 2514-2523, Nov 2007.

[69] C. E. Tabit, W. B. Chung, N. M. Hamburg and J. A. Vita, "Endothelial dysfunction in diabetes mellitus: Molecular mechanisms and clinical implications," Reviews in Endocrine and Metabolic Disorders, vol. 11, no. 1, pp. 61-74, Feb 2010.

[70] A. Risso, F. Mercuri, L. Quagliaro, G. Damante and A. Ceriello, "Intermittent high glucose enhances apoptosis in endothelial cells in culture," American Journal of Physiology - Endocrinology and Metabolism, vol. 281, no. 5, pp. E924E930, Nov 2001.

[71] A. M. Schmidt, O. Hori, J. X. Chen, J. F. Li, J. Crandall, J. Zhang, R. Cao, S. D. Yan, J. Brett and D. Stern, "Advanced Glycation Endproducts Interacting with their Endothelial Receptor Induce Expression of Vascular Cell Adhesion Molecule-1 (VCAM-1) in Cultured Human Endothelial Cells and in Mice: A potential mechanism for the accerlated vasculopathy of diabetes," Journal of Clinical Investigation, vol. 96, no. 3, pp. 1395-1403, Sep 1995.

[72] G. Arcaro, A. Cretti, S. Balzano, A. Lechi, M. Muggeo, E. Bonora and R. C. 
Bonadonna, "Insulin Causes Endothelial Dysfunction in Humans," Circulation, vol. 105, pp. 576-582, Feb 2002.

[73] K. Kubozi, Z. Y. Jiang, N. Takahara, S. W. Ha, M. Igarashi, T. Yamauchi, E. P. Feener, T. P. Herbert, C. J. Rhodes and G. L. King, "Regulation of Endothelial Constitutive Nitric Oxide Synthase Gene Expression in Endothelial Cells and In Vivo: a specific vascular action of insulin," Circulation, vol. 101, no. 6, pp. 676-681, Feb 2000.

[74] R. Edmonson, J. J. Broglie, A. F. Adcock and L. Yang, "Three-Dimensional Cell Culture Systems and their Applications in Drug Discovery and Cell-Based Biosensors," Assay Drug Dev Technol, vol. 12, no. 4, pp. 207-218, 2014.

[75] F. M. Ho, W. W. Lin, B. C. Chen, C. M. Chao, C.-R. Yang, L. Y. Lin, C. C. Lai, S. H. Liu and C. S. Liau, "High glucose-induced apoptosis in human vascular endothelial cells is mediated through NF-kB and c-Jun NH2-terminal kinase pathway and prevented by PI3K/Akt/eNOS pathway," Cellular Signaling, vol. 18, no. 3, pp. 391-399, March 2006. 


\section{APPENDICES}

\section{APPENDIX A: Immunofluorescence Staining Protocol for PECAM}

Materials:

- Samples

- Pap pen

- $\quad$ PBS (phosphate buffered saline)

- Saponin

- $\quad$ FBS (fetal bovine serum)

- Primary antibody - mouse anti-CD31 (PECAM)

- Waste beakers

\section{Staining Protocol}

1. Label slides and treatments

2. Prepare about $50 \mathrm{ml}$ of PBS

3. Using the pap pen, draw a thick circle around the sample and let dry for 5 minutes, or until dry

4. Gently apply PBS using a micropipette and wash (2-3 x 5 min)

5. Permeabilize cells using saponin (1\%) for 10 minutes

6. Gently apply and wash in PBS (1 x 5 min)

7. Make $10 \%$ serum with PBS

8. Block for 60 minutes at room temperature

9. Make primary antibody solution (1:500 blocking solution)
- Secondary antibody - AlexaFluor 488 goat anti-mouse

- Tin Foil

- $\quad$ BBI (bisbenzimide)

- Mounting media

- Cover slips

- Pipettes and tips 
10. Remove blocking and incubate in primary antibody overnight ( 8-10 hours) in the fridge

11. Wash in PBS (3 x $5 \mathrm{~min})$

12. Make secondary antibody solution $(5 \mu \mathrm{g} / \mathrm{ml})$ in blocking solution

13. Incubate in secondary antibody for 45-60 minutes at room temperature
a. WRAP IN FOIL

14. Wash in PBS (3X 5 min)

a. Don't forget foil

15. BBI (1:400) for 5 minutes

16. Wash in PBS for 5 min

17. Mount or prepare for observation

18. Fluorescence: PECAM shows up in $488(3,2)$ and BBI in $400(1,1)$. 


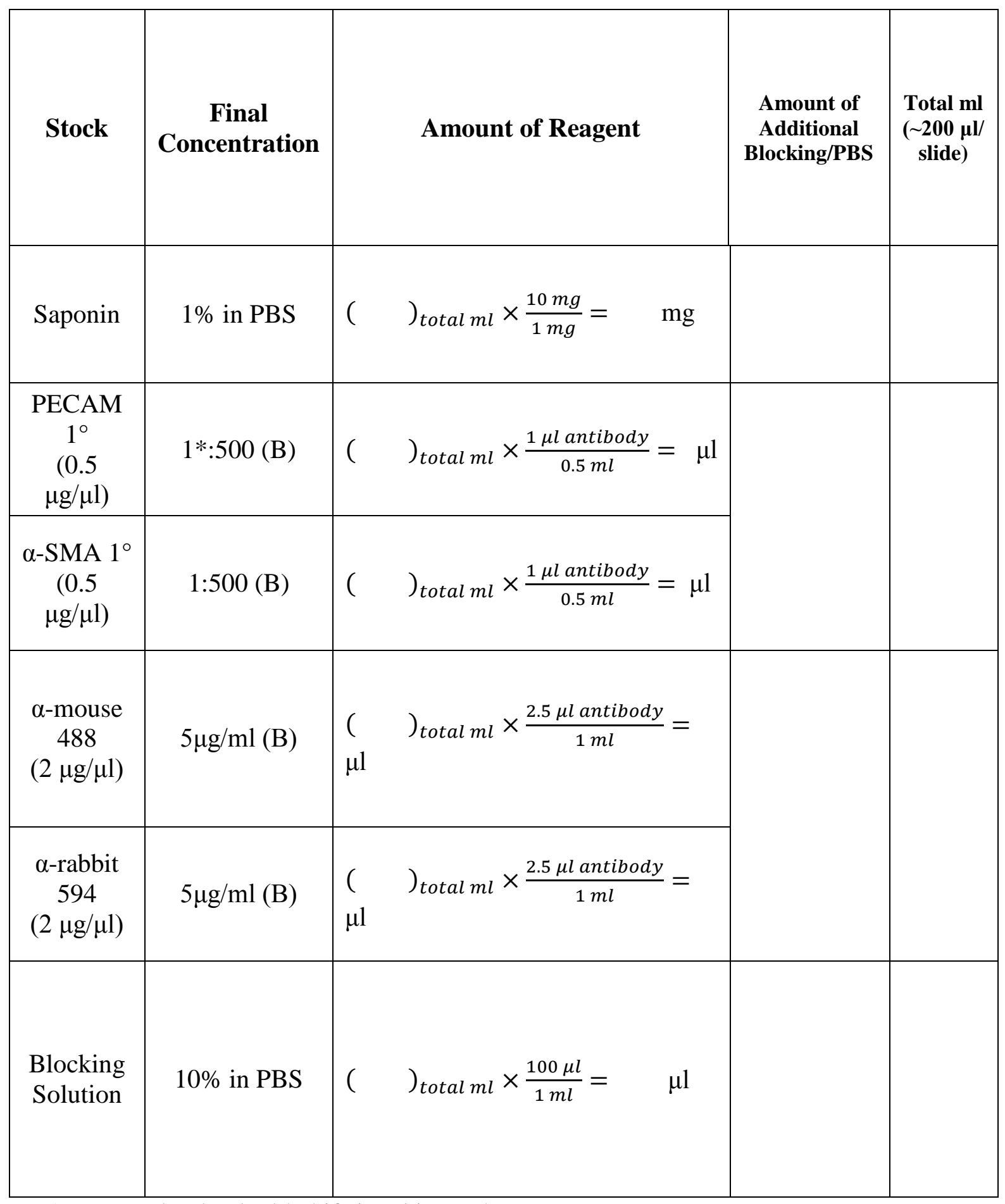

*May need to be doubled if signal is weak 


\section{APPENDIX B: Protocol for RNA Isolation from Cultured Cells and BVM Samples (SOP7100)}

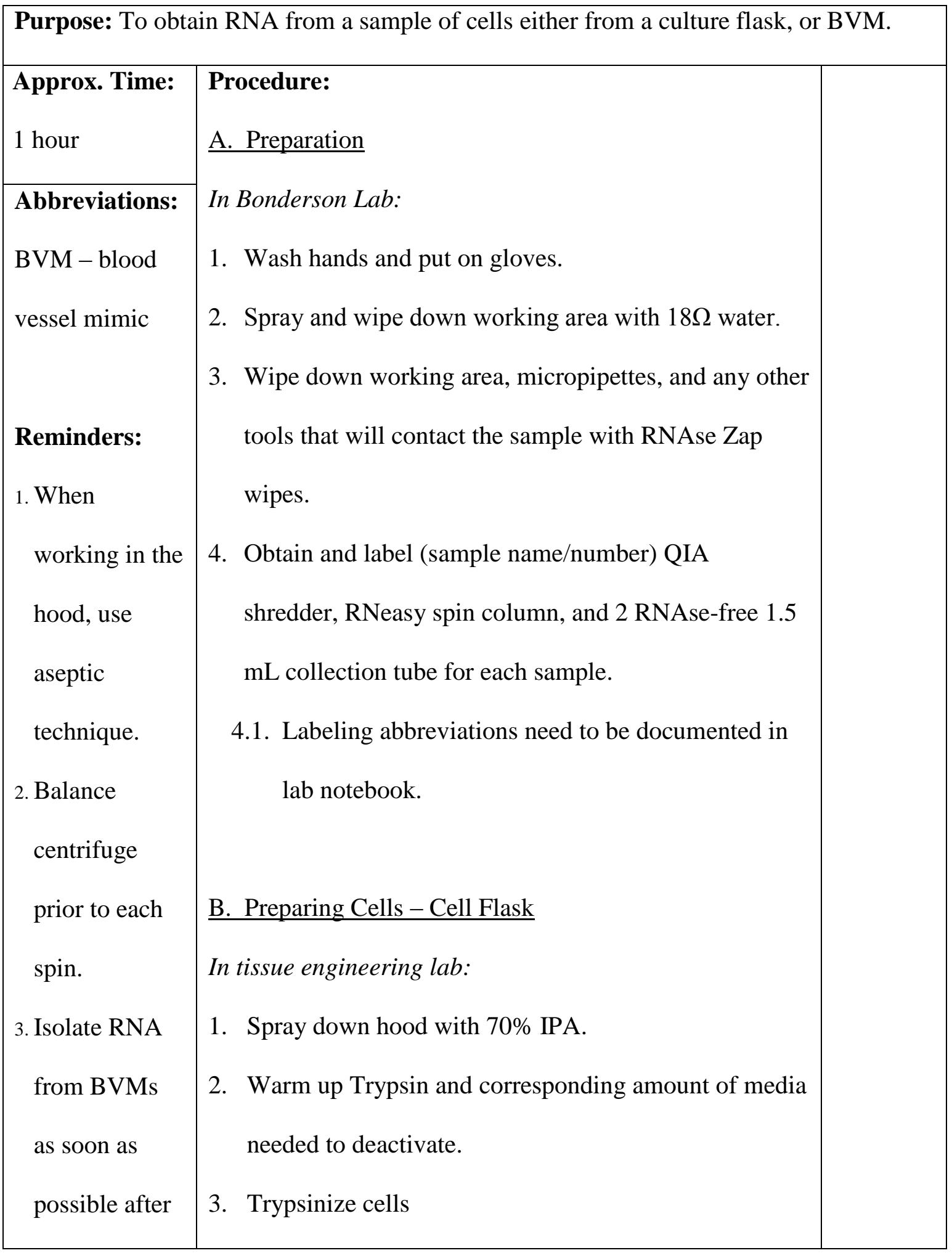




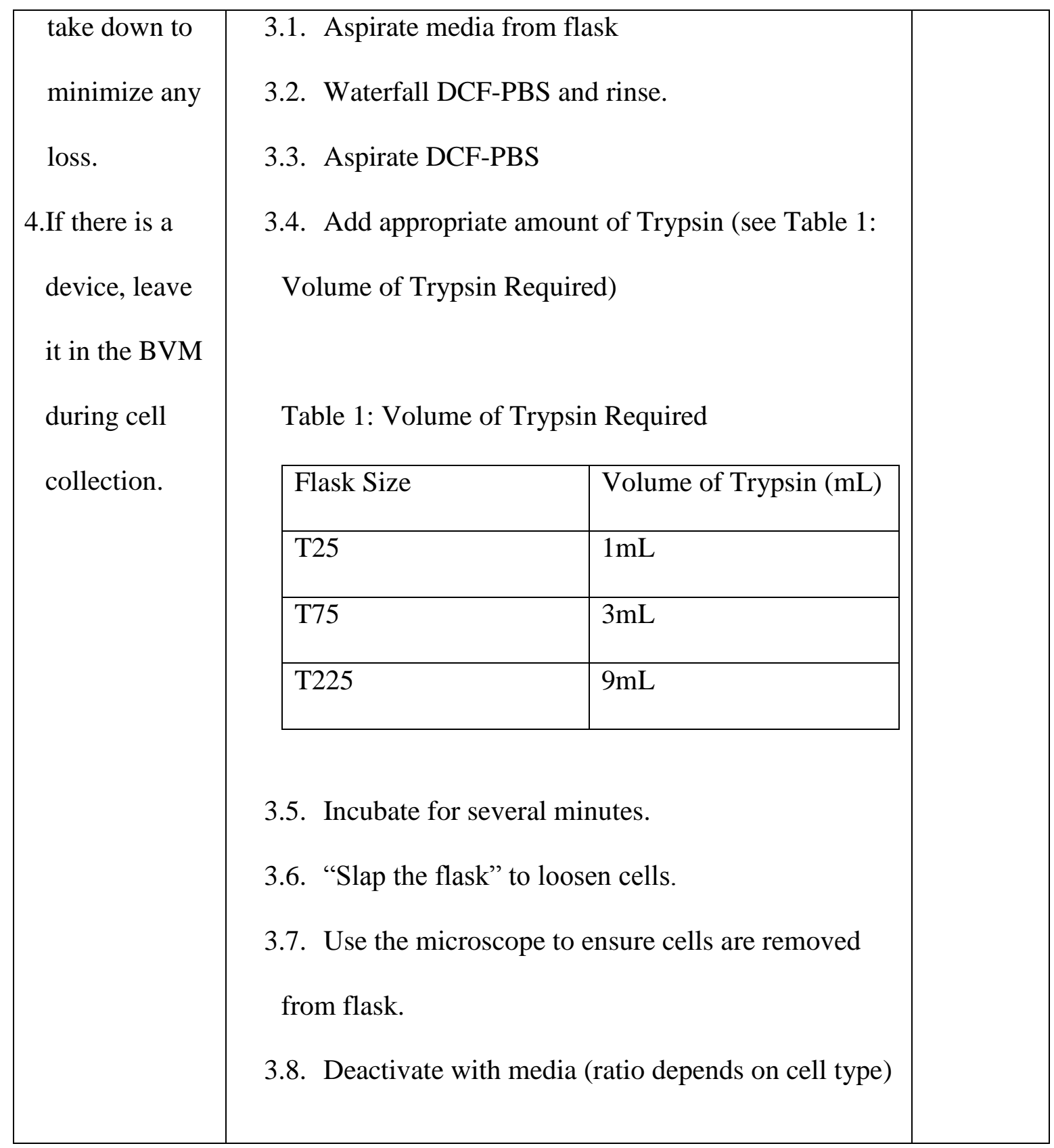




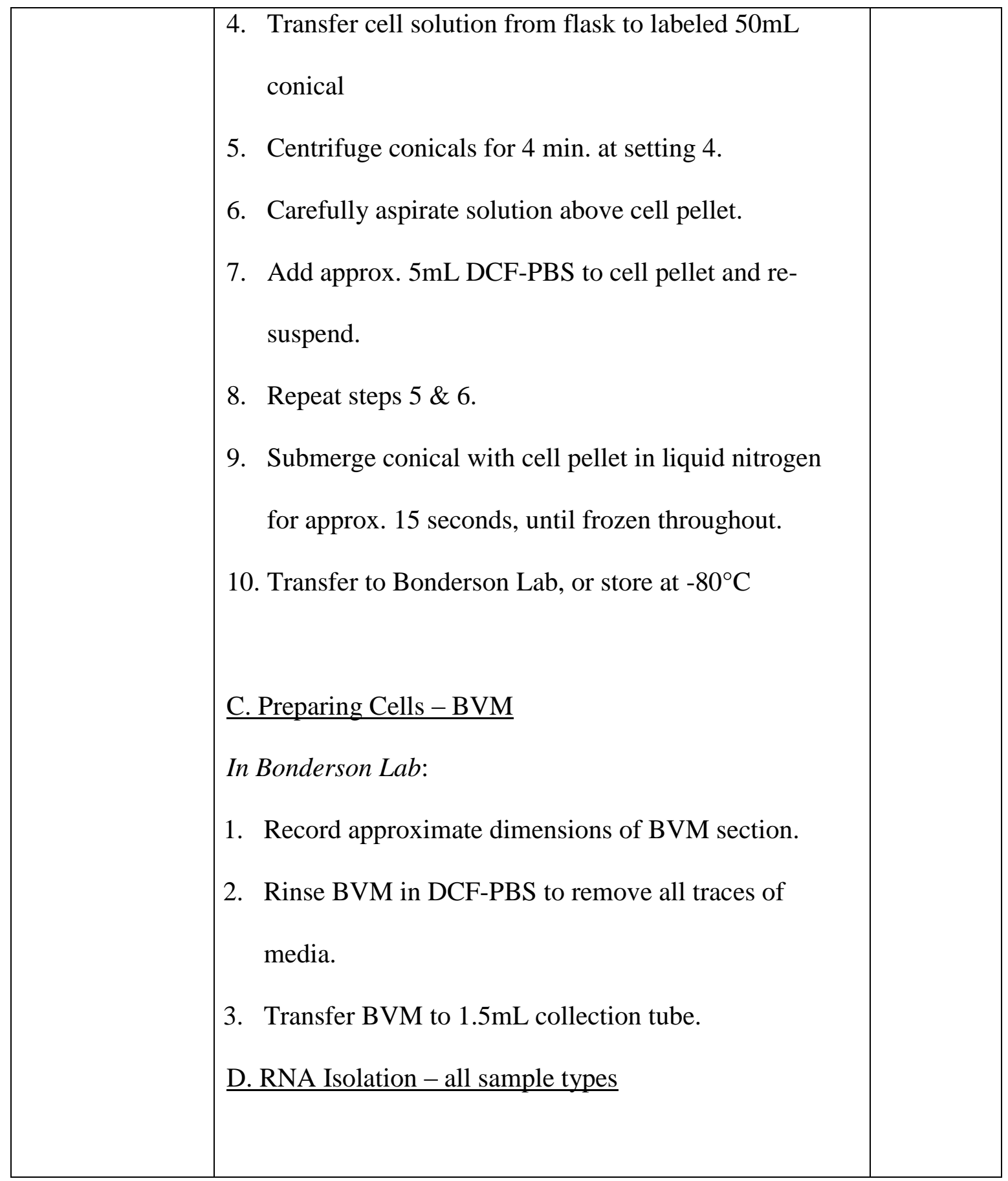




\begin{tabular}{|c|c|}
\hline \multirow{2}{*}{$\begin{array}{l}\text { Materials: } \\
\text { 1. Trypsin \& cell }\end{array}$} & In Bonderson Lab: \\
\hline & 1. Add $600 \mathrm{uL}$ of Buffer RLT to sample (see Table 2 ). \\
\hline media (for cell & 1.1. Pipette up and down to mix. \\
\hline flask & 1.2. Vortex tube for 2 minutes. \\
\hline isolation) & *Note: For BVMs, add half the volume of Buffer RLT \\
\hline 2. Sterile pipet & and perform step 1 twice. \\
\hline tips & \\
\hline 3. RNEasy Mini & 2. Transfer all cell lysate to QIA shredder spin column. \\
\hline Kit (Qiagen) & 2.1. Centrifuge at $16.1 \times 10^{4} \mathrm{rcf}$ for 2 minutes. \\
\hline - RNeasy & 3. Add $600 \mathrm{uL}$ of $70 \%$ ethanol to flow through from QIA \\
\hline spin & shredder spin column. \\
\hline columns & 3.1. Pipette up and down to mix. \\
\hline - QIA & 4. Transfer $600 \mathrm{uL}$ of flow through solution to RNeasy \\
\hline shredder & spin column. \\
\hline spin & 4.1. Centrifuge at $16.1 \times 10^{4} \mathrm{rcf}$ for 30 seconds. \\
\hline columns & 4.2. Discard flow through in waste. \\
\hline$-\quad 1.5 \mathrm{~mL}$ & 4.3. Repeat step 4 for remaining flow through from \\
\hline \&/or & QIA shredder spin column. \\
\hline $2.0 \mathrm{~mL}$ & 5. Add 700uL RW 1 washing buffer to RNeasy spin \\
\hline collection & column \\
\hline tubes & 5.1. Centrifuge at $16.1 \times 10^{4} \mathrm{rcf}$ for 30 seconds. \\
\hline - $\quad$ Buffer & 5.2. Discard flow through in waste. \\
\hline RLT & 6. Add 500uL Buffer RPE to RNeasy spin column \\
\hline
\end{tabular}




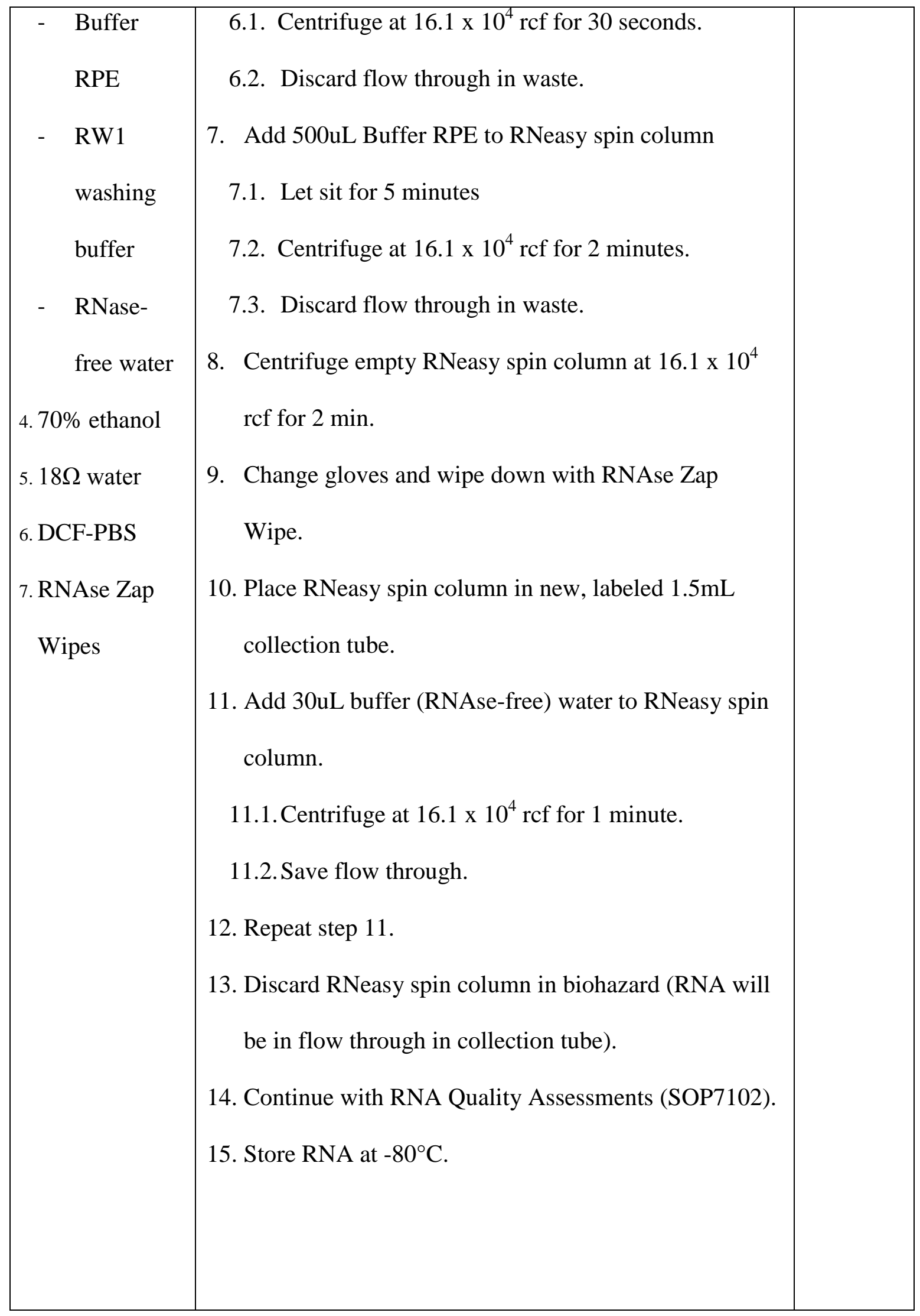




\begin{tabular}{|l|l|}
\hline $\begin{array}{l}\text { E. Clean Up } \\
\text { 1. Discard remaining waste. } \\
\text { 1.1.Spin columns, collection tubes, and pipette tips go in } \\
\text { biohazard. } \\
\text { 2. Return supplies and reagents to proper location. } \\
\text { 3. Clean working area with } 70 \% \text { IPA. }\end{array} \mid$ & $\mid$
\end{tabular} \mid

\begin{tabular}{|l|l|l|}
\hline \multicolumn{2}{|l|}{ Revision History } \\
\hline A & Michael Gibbons & 120608 \\
& Sarah Ur & \\
\hline B & Tristin McGuffick & 1501222 \\
\hline C & Shelby Kunz & 160601 \\
\hline
\end{tabular}




\section{APPENDIX C: Protocol for RNA Assessment (SOP7102)}

\begin{tabular}{|c|c|}
\hline asses & e quality and determine concentration of isolated $\mathrm{R}$ \\
\hline $\begin{array}{l}\text { Approx. Time: } \\
10 \text { minutes/sample }\end{array}$ & $\begin{array}{l}\text { Procedure: } \\
\text { In Bonderson lab: } \\
\text { A. Spectrophotometry - Nanodrop } 2000\end{array}$ \\
\hline Abbreviations: & 1. Open NanoDrop 2000 program on laptop \\
\hline EtBr - Ethidium & 2. Select Nucleic Acid \\
\hline bromide & 3. Perform wavelength verification \\
\hline TAE - Trisacetate & 3.1. Remove Kim Wipe and lower arm \\
\hline EDTA & 4. Select RNA as sample type \\
\hline Reminders: & 5. Rinse stage with RNAse-free water \\
\hline 1. Remove gloves & 6. Blank spectrophotometer \\
\hline when using & 6.1. Add 1uL RNAse-free water to the stage \\
\hline NanoDrop 2000 & 6.2. Lower arm and select "blank" \\
\hline laptop. & 7. Dry stage gently with Kim wipe \\
\hline 2. Mark orientation & 8. Name sample (using normal naming convention) \\
\hline of samples in & 9. Add $1 \mathrm{uL}$ of sample RNA to stage \\
\hline electrophoresis & 10. Lower arm and click "measure" \\
\hline tray. & 10.1. Record concentration, 260/280, and 260/230 \\
\hline
\end{tabular}




\begin{tabular}{|c|c|}
\hline Materials: & values. \\
\hline 1. DNAse-free & 10.2. Repeat measurement twice more (total 3 \\
\hline pipet tips & values for each sample) \\
\hline 2. $1 \mathrm{uL}$ pipet & 11. Gently dab stage dry after use. \\
\hline 3. Agarose & 11.1.Leave Kim wipe under arm when finished. \\
\hline 4. TAE & \\
\hline 5. $\mathrm{EtBr}$ & In Bonderson or Peterson lab: \\
\hline 6. Flask \& & B. Gel Electrophoresis \\
\hline graduated & 1. Measure $50 \mathrm{~mL}$ TAE \\
\hline cylinder & 2. Weigh $0.5 \mathrm{~g}$ Agarose in weigh boat \\
\hline 7. RNAse-free & 3. Add Agarose to TAE and microwave in 30 second \\
\hline water & intervals until Agarose has dissolved \\
\hline 8. Kim Wipes & 3.1. Swirl between intervals \\
\hline 9. Loading dye & 3.2. Watch carefully to avoid boil over \\
\hline 10. DNA ladder & 4. Add $2 \mathrm{uL}$ of EtBr to TAE \& Agarose solution \\
\hline 11.Parafilm & 5. Let TAE \& Agarose solution cool \\
\hline & 5.1. Until you can hold your palm to the flask for \\
\hline & $10 \mathrm{sec}$. \\
\hline Equipment: & 6. Tape sides of electrophoresis tray to form mold \\
\hline 1. Electrophoresis & 7. Pour $\mathrm{TAE} / \mathrm{EtBr} \&$ Agarose solution into mold \\
\hline tray & 7.1. Place comb into mold \\
\hline 2. Gel comb & 7.2. Wait for TAE solution to polymerize \\
\hline 3. Scale & 7.3. Remove comb \\
\hline
\end{tabular}




\begin{tabular}{|c|c|}
\hline $\begin{array}{l}\text { 4. UV machine \& } \\
\text { camera }\end{array}$ & $\begin{array}{l}\text { 8. Mix sample and loading dye on parafilm or in } \\
\text { tubes. } \\
\text { 8.1. } 2-5 \mathrm{uL} \text { of sample }+2 \mathrm{uL} \text { loading dye } \\
\text { 8.2. } 2 \mathrm{uL} \text { of DNA ladder }+2 \mathrm{uL} \text { loading dye } \\
\text { 9. Pipet samples into gel wells } \\
\text { 10. Fill electrophoresis tray with TAE. } \\
\text { 10.1. Add } 1 \mathrm{uL} \text { of EtBr to positive (red) pool in tray. } \\
\text { 11. Run at } 100 \mathrm{~V} \text { for } 1 \text { hour, checking periodically } \\
\text { 12. Turn off power. } \\
\text { 13. Remove gel from tray. } \\
\text { 14. Place gel in UV imaging gel dock. } \\
\text { 15. Turn on camera and gel dock. } \\
\text { 16. Obtain image using filter \# } 3 \text { on gel dock. }\end{array}$ \\
\hline
\end{tabular}

\begin{tabular}{|l|l|l|}
\hline \multicolumn{2}{|l|}{ Revision History } \\
\hline A & Michael Gibbons & 120608 \\
& Sarah Ur & \\
\hline B & Tristin McGuffick & 140523 \\
\hline C & Shelby Kunz & 160601 \\
\hline
\end{tabular}




\section{APPENDIX D: Protocol for Reverse Transcription (SOP7103)}

\begin{tabular}{|c|c|}
\hline Purpose: To obt & cDNA via reverse transcription of isolate mRNA. \\
\hline Approx. Time: & Procedure: \\
\hline 1 hour (for & $\underline{\text { A. Preparation }}$ \\
\hline approx. 10 & 1. Using average concentration obtained in RNA \\
\hline samples) & assessment, calculate volume of RNA needed. \\
\hline Abbreviations: & 1.1. Determine amount of RNA (ng) for duration of \\
\hline $\mathrm{RT}$ - reverse & experiment. \\
\hline transcriptase & 1.2. $200 \mathrm{ng}$ to $1000 \mathrm{ng}$ are needed to run $\mathrm{qPCR}$ \\
\hline & 1.3. $(\mathrm{x}$ ng of RNA $) *(1 \mathrm{~mL} /[\mathrm{RNA} n g / \mathrm{nL}])=$ Volume \\
\hline Reminders: & RNA (uL) \\
\hline 1. Keep working & 2. Calculate amount of nuclease free water needed. \\
\hline area and tools & 2.1. $15 \mathrm{uL}-$ Volume RNA $=$ Volume $\mathrm{H}_{2} \mathrm{O}(\mathrm{uL})$ \\
\hline RNAse free & 3. Wipe down working area, micropipettes, and any other \\
\hline (wipe down & tools with RNAse Zap Wipes. \\
\hline tools & \\
\hline continually). & B. iScript Transcription \\
\hline 2. Store iScript & 1. Prepare master mix \\
\hline cDNA & 1.1. $4 \mathrm{uL}$ of $5 \mathrm{X}$ iScript Reaction mix per sample \\
\hline synthesis kit at & 1.2. $1 \mathrm{uL}$ of RT per reaction \\
\hline$-20^{\circ} \mathrm{C}$. & $\begin{array}{l}\text { 1.3. Include additional } 10 \% \text { of each reagent for pipetting } \\
\text { error }\end{array}$ \\
\hline
\end{tabular}




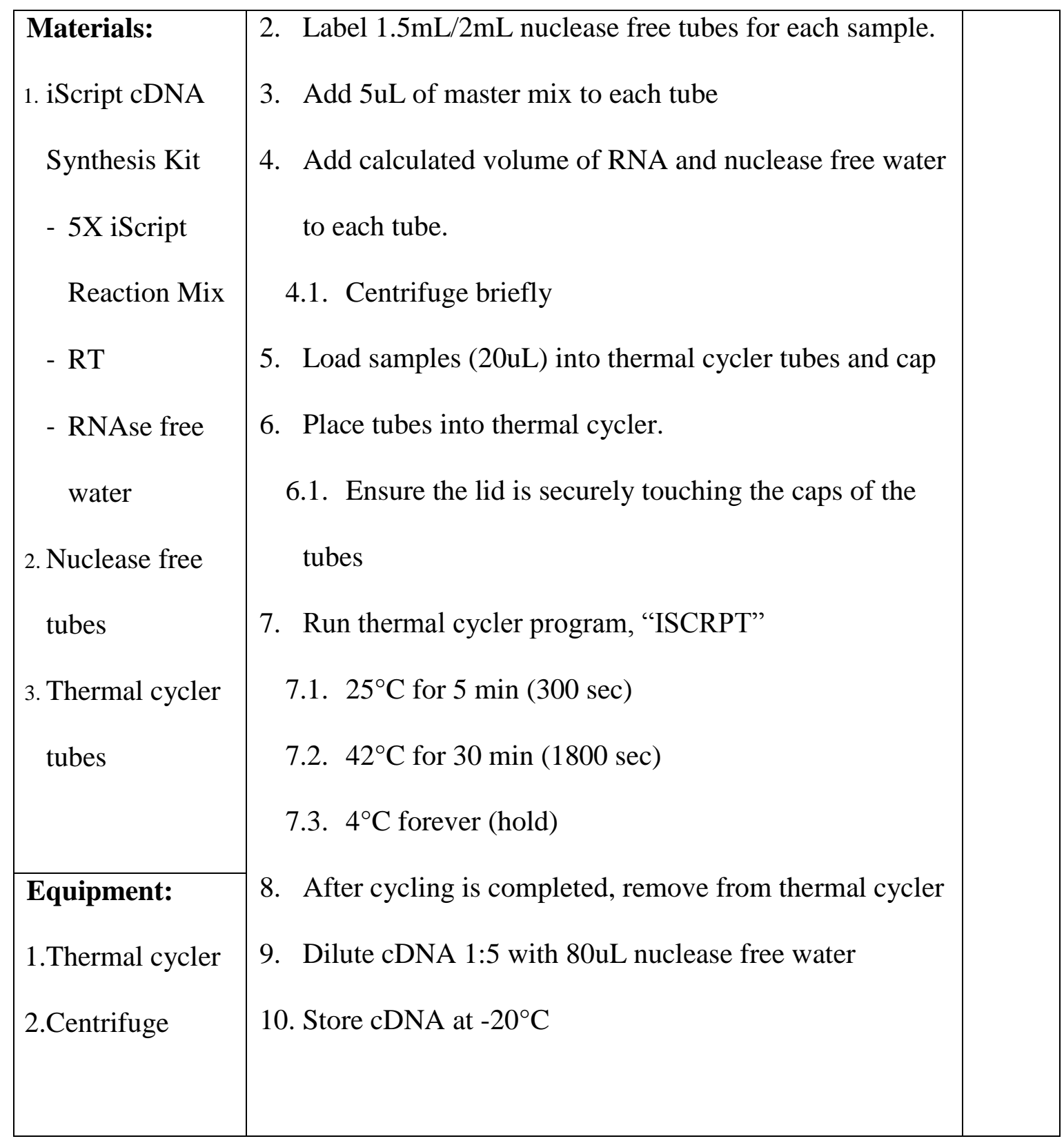

\begin{tabular}{|l|l|l|}
\hline \multicolumn{2}{|l|}{ Revision History } \\
\hline A & $\begin{array}{l}\text { Michael Gibbons } \\
\text { Sarah Ur }\end{array}$ & 120608 \\
\hline B & Tristin McGuffick & 140605 \\
\hline C & Shelby Kunz & 160601 \\
\hline
\end{tabular}




\section{APPENDIX E: Protocol for qPCR (SOP7105)}

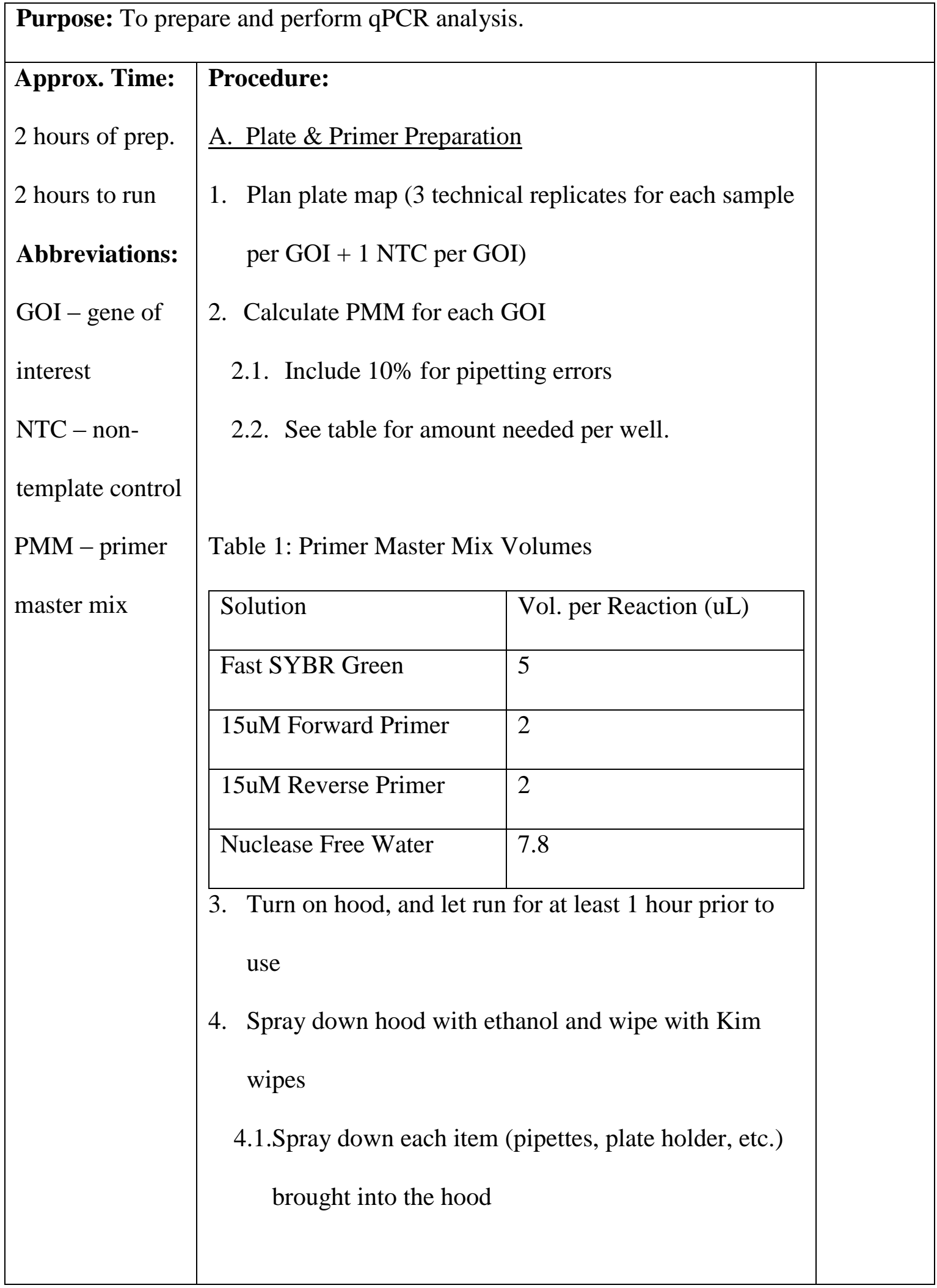




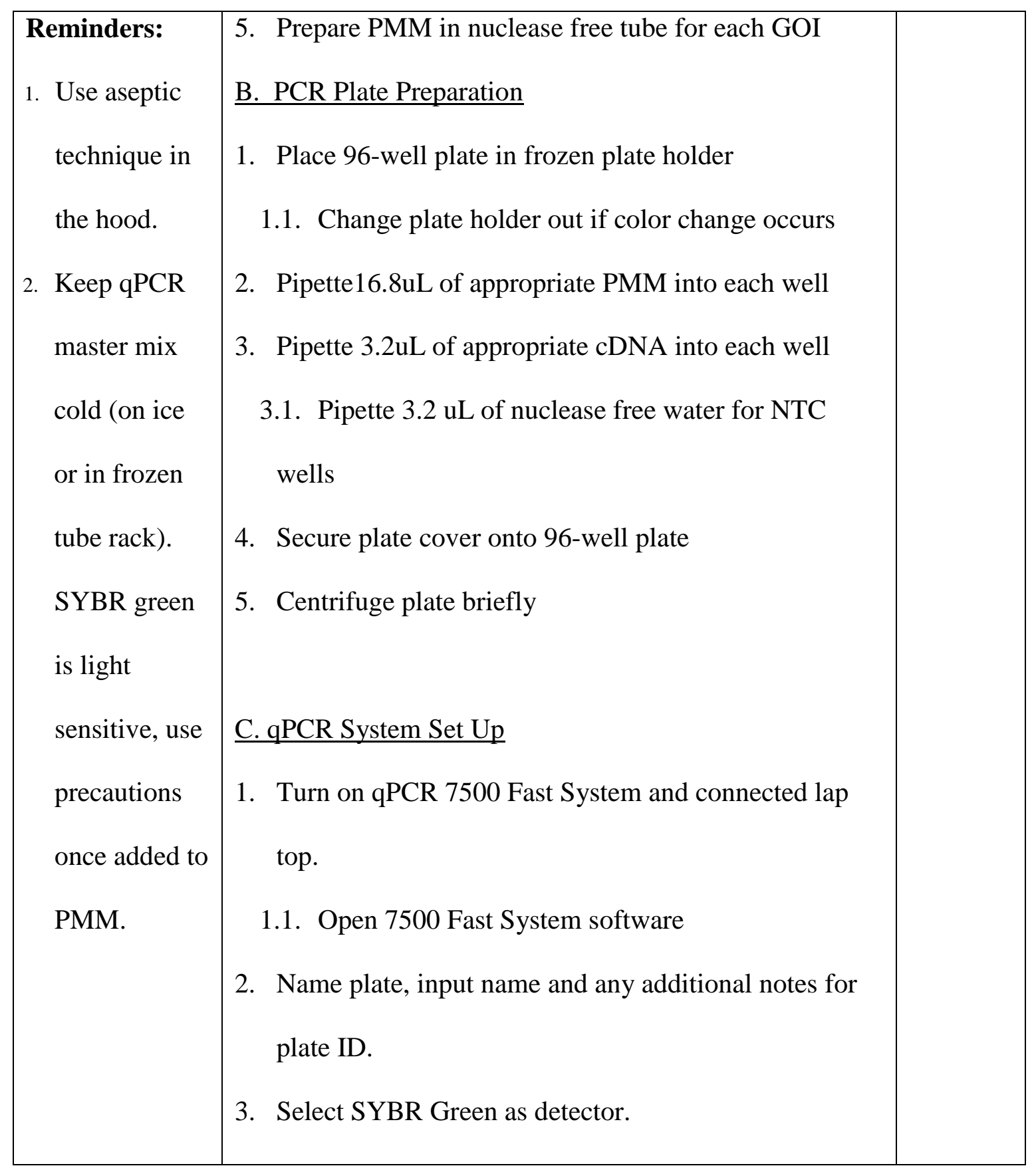




\begin{tabular}{|c|c|c|c|}
\hline Materials: & \multicolumn{3}{|c|}{ 3.1. Use ROX as passive reference } \\
\hline $\begin{array}{l}\text { 1. Fast SYBR } \\
\text { Green }\end{array}$ & $\begin{array}{l}\text { 4. Input sam } \\
\text { data analy }\end{array}$ & and GOI & well (will help with \\
\hline 2. Fast 96-well & \multicolumn{3}{|c|}{ 4.1. Select action for each well: Standard (for samples), } \\
\hline qPCR plate & \multicolumn{3}{|c|}{ NTC, or none (for empty wells) } \\
\hline 3. Plate cover & \multicolumn{3}{|c|}{ 5. Place plate in qPCR 7500 Fast System } \\
\hline 4. $15 \mathrm{uM}$ & \multicolumn{3}{|c|}{ 6. Use standard run times as listed in table 2.} \\
\hline primers & \multicolumn{3}{|c|}{ 6.1. Include a dissociation cycle to the run. } \\
\hline 5. Nuclease free & & & \\
\hline water & \multicolumn{3}{|c|}{ Table 2: Thermal Cycling Times for qPCR 7500 Fast } \\
\hline 6. Frozen PCR & \multicolumn{3}{|l|}{ System } \\
\hline plate holder & Temperature & Duration & Cycle \\
\hline 7. Ethanol & $95.0^{\circ} \mathrm{C}$ & 20 secs & HOLD \\
\hline 8. Kim wipes & $95.0^{\circ} \mathrm{C}$ & 3 secs & HOLD \\
\hline 9. Micropipettes & $60.0^{\circ} \mathrm{C}$ & 30 secs & 40 \\
\hline Nuclease free & \multicolumn{3}{|c|}{ Dissociation Cycle (Standard Settings) } \\
\hline pipette tips & & & \\
\hline
\end{tabular}




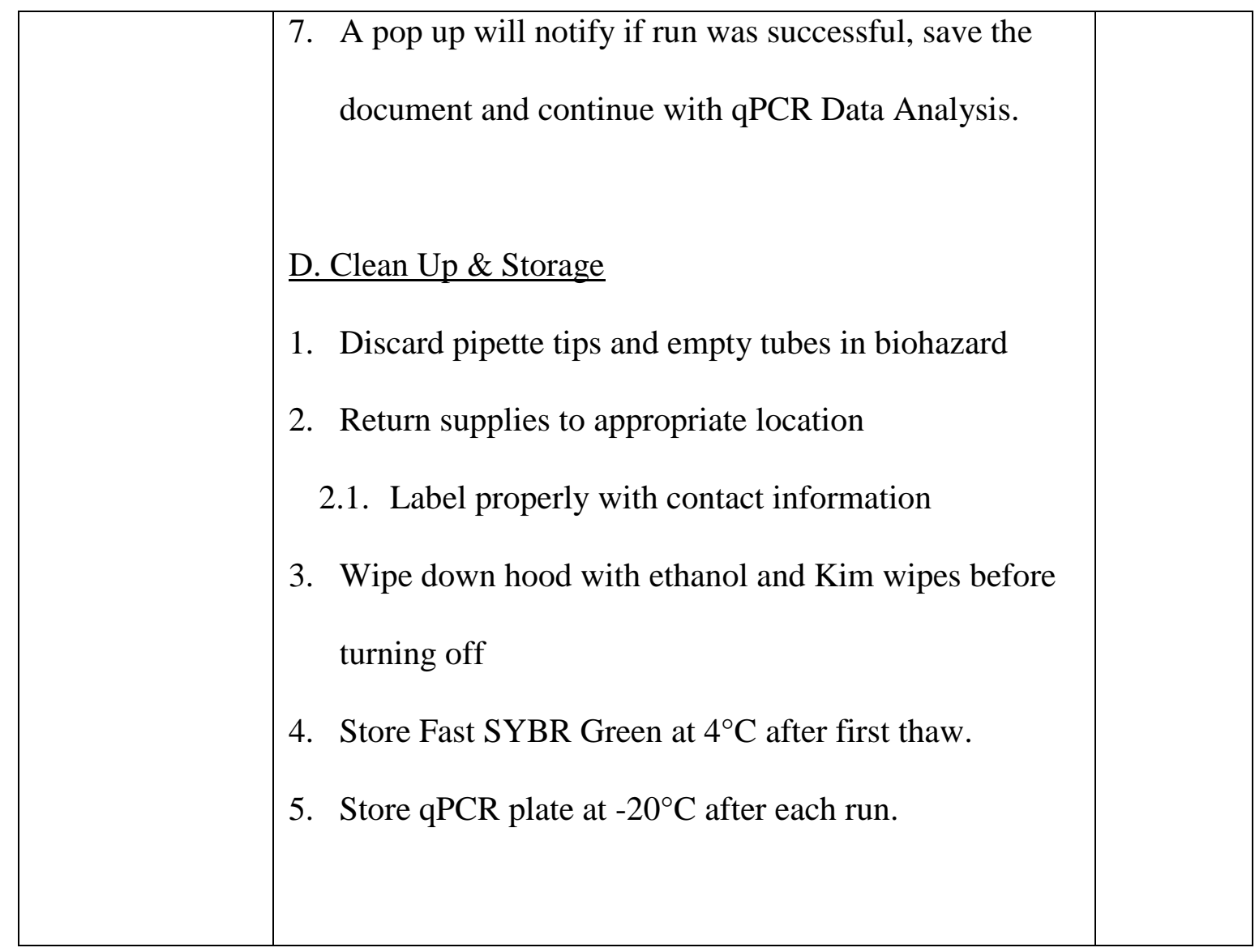

\begin{tabular}{|l|l|l|}
\hline \multicolumn{2}{|l|}{ Revision History } \\
\hline A & Michael Gibbons & 120608 \\
& Sarah Ur & \\
\hline B & Tristin McGuffick & 140605 \\
\hline C & Shelby Kunz & 160601 \\
\hline
\end{tabular}




\section{APPENDIX F: Protocol for qPCR Data Analysis (SOP7106)}

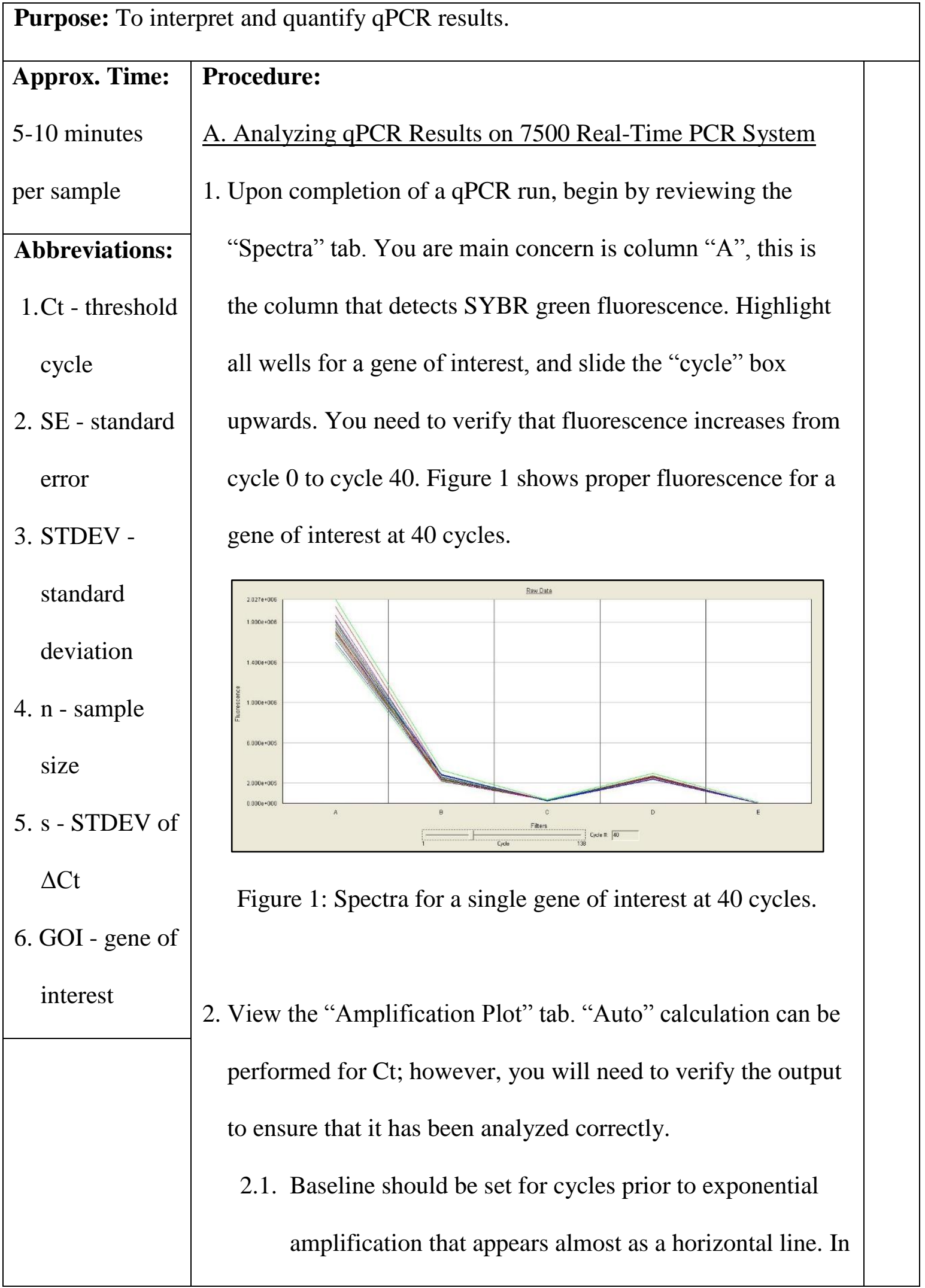




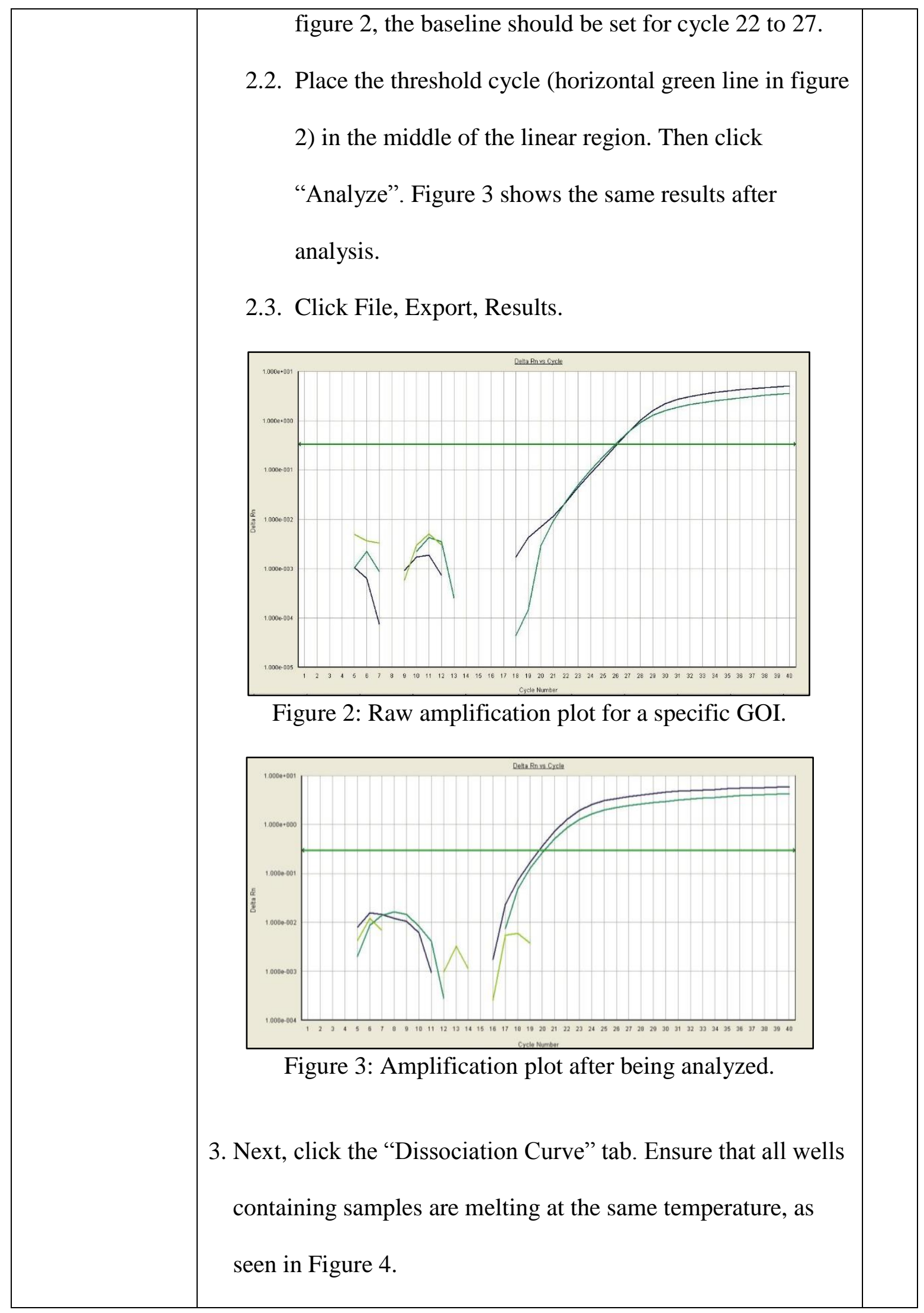




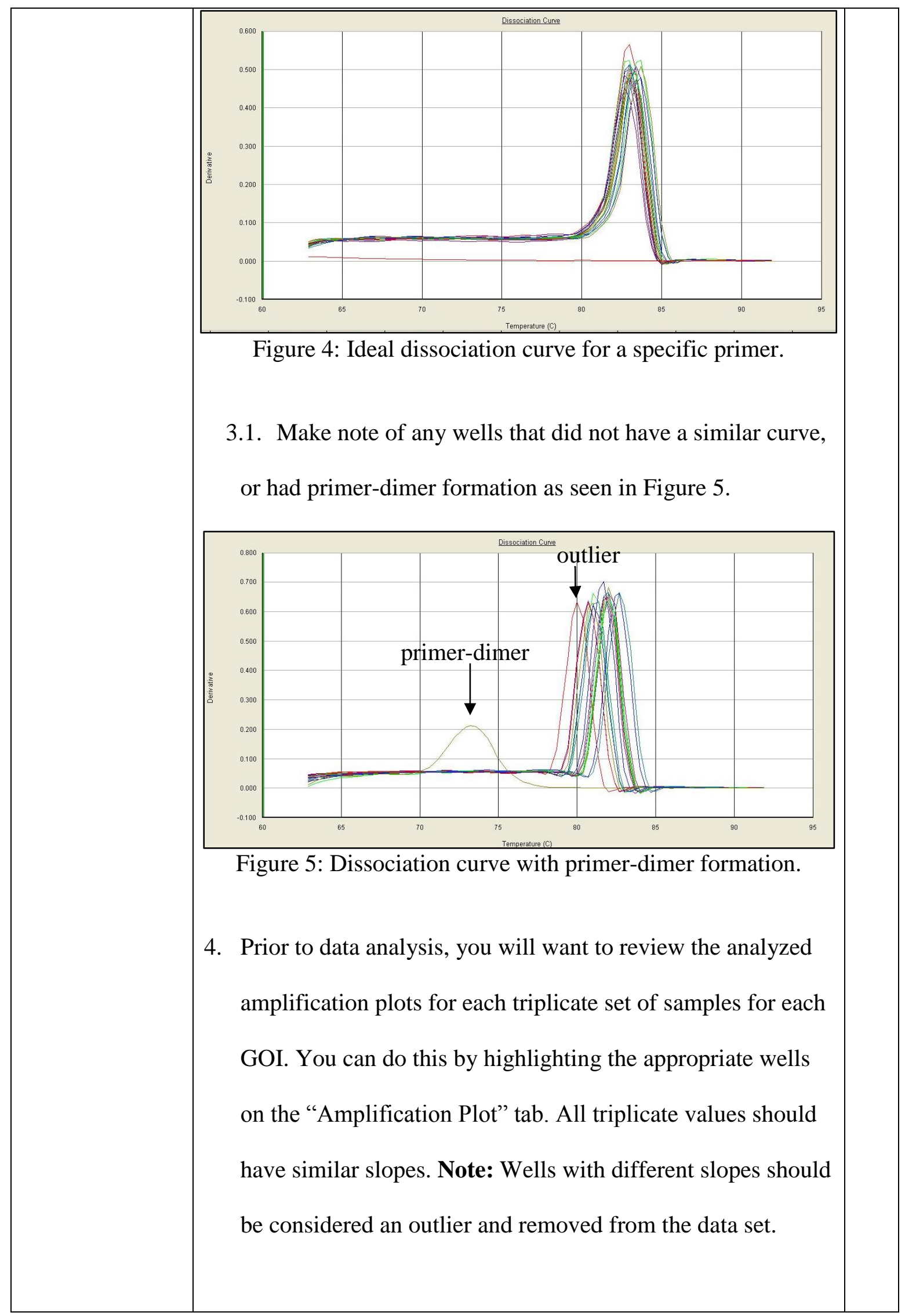


5. Lastly, you will need to review the dissociation curve for outliers and primer-dimer formation, and remove data as necessary.

\section{B. Reference Gene Validation}

1. Average triplicate $\mathrm{Ct}$ values for each sample for the reference gene.

2. Plot average $\mathrm{Ct}$ values for each sample group on a bar chart.

2.1. Calculate SE using the following equation for the error bars:

$$
S E=\frac{S T D E V}{\sqrt{n}}
$$

3. Perform a one-way ANOVA with a 95\% confidence interval to ensure there is no significant difference between samples for the reference gene.

3.1. If $\mathrm{p}<0.05$ the reference gene cannot be used for the samples.

\section{Relative Expression Analysis}

1. Average triplicate $\mathrm{Ct}$ values for each sample/gene of interest set.

2. Normalize samples to the appropriate reference gene using the following equation:

$$
\Delta C t=C t_{\text {sample }, G O I}-C t_{\text {sample, reference }}
$$

3. To obtain $\Delta \Delta \mathrm{Ct}$ data, an experimental and control group must 


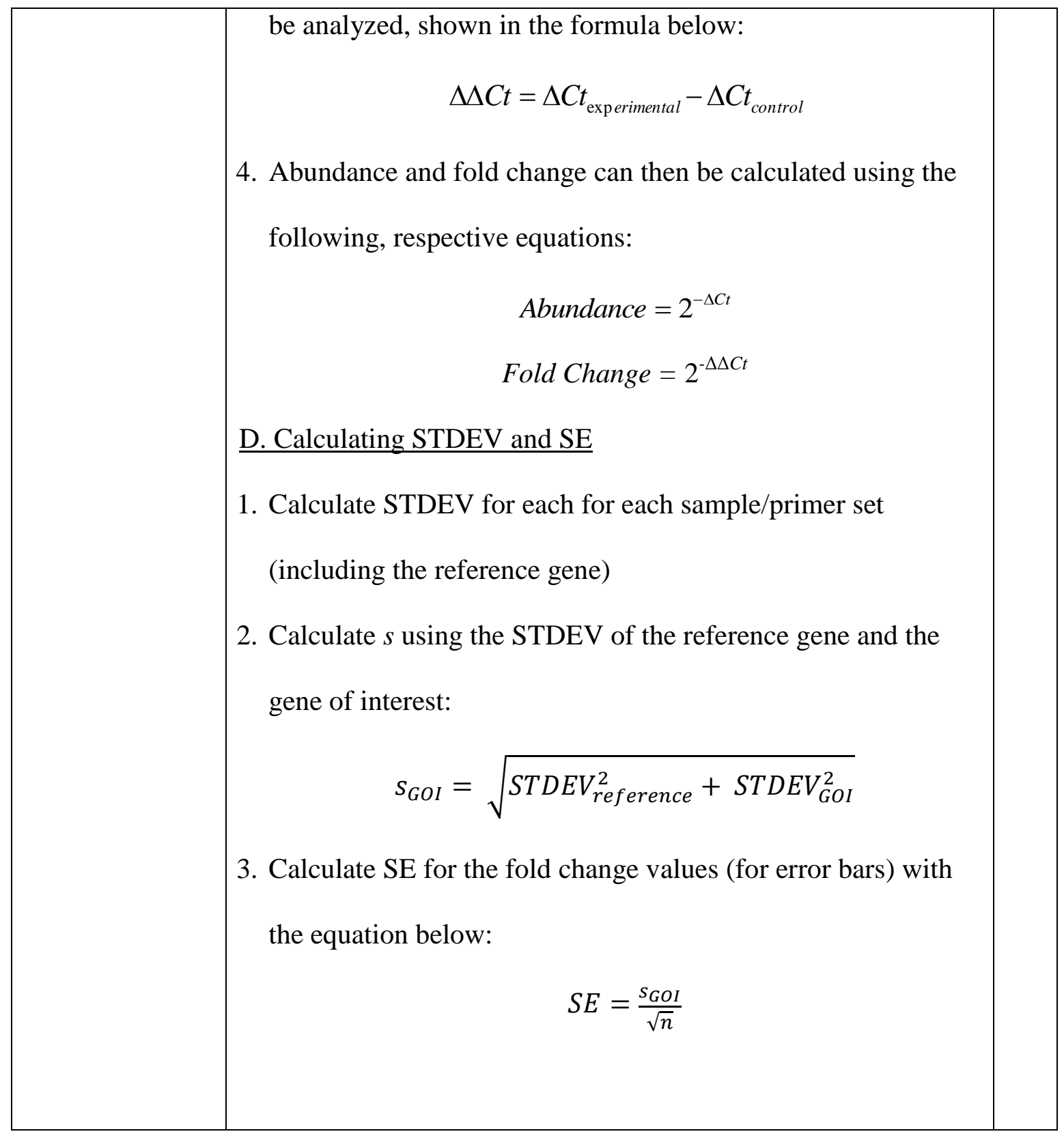

\begin{tabular}{|l|l|l|}
\hline \multicolumn{2}{|l|}{ Revision History } \\
\hline A & $\begin{array}{l}\text { Michael Gibbons } \\
\text { Sarah Ur }\end{array}$ & 120608 \\
\hline B & Tristin McGuffick & 140301 \\
\hline C & Shelby Kunz & 160601 \\
\hline
\end{tabular}




\section{APPENDIX G: Protocol for Primer Design (SOP7104)}

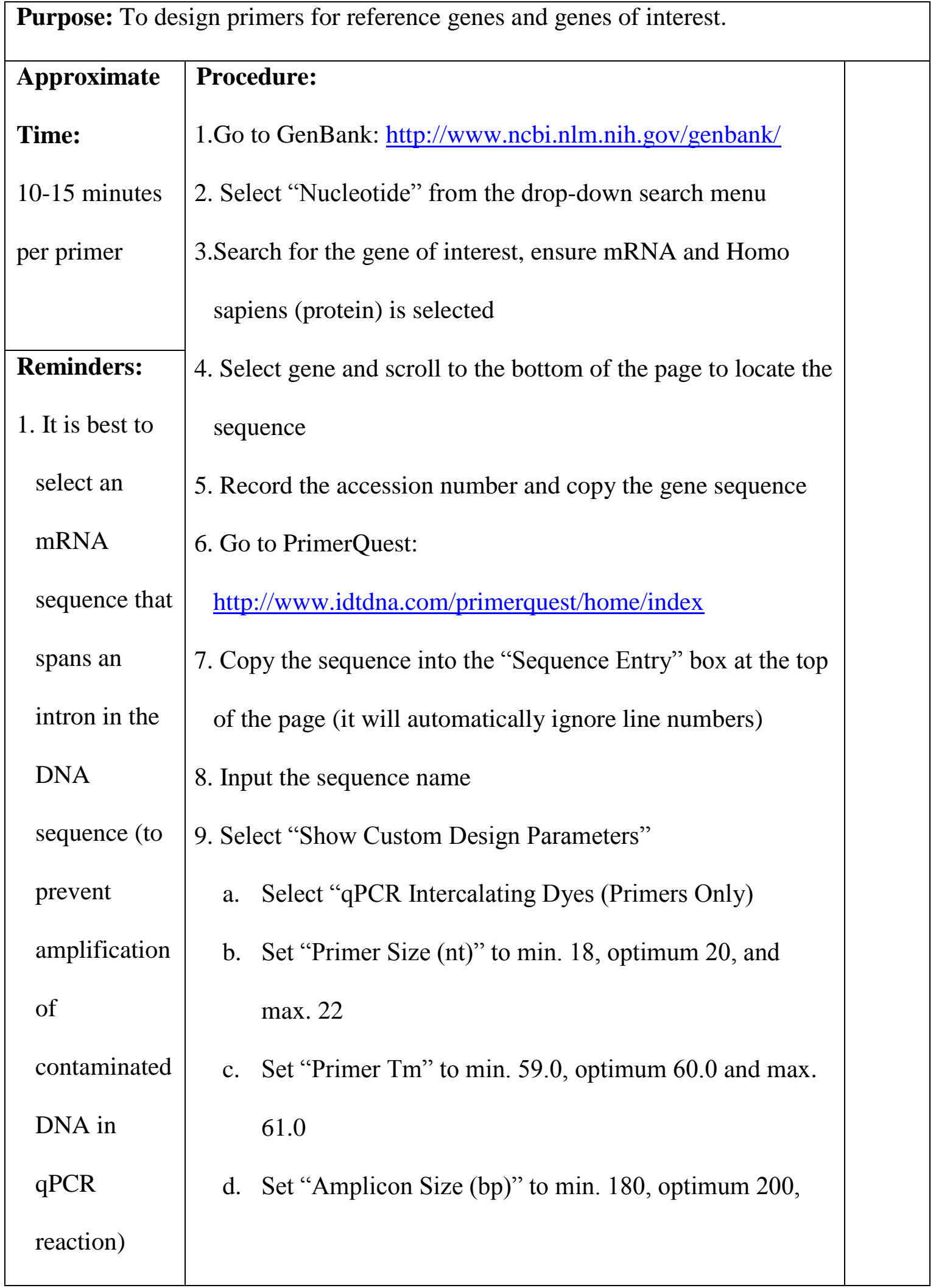




\begin{tabular}{|c|c|}
\hline 2. The first & and max. 220 \\
\hline primer set is & e. Leave all other parameters as is \\
\hline not always & f. Click "Get Assays," PrimerQuest will output 5 sets of \\
\hline the best, & forward and reverse primers that match input criteria \\
\hline complete all & g. Select "View Assay Details" for the desired primer set. \\
\hline primer & This will open a new page with the forward and reverse \\
\hline selection & primers. Copy these sequences. \\
\hline steps for & 10. Go to NetPrimer (leaving the IDT page open): \\
\hline multiple & http://www.premierbiosoft.com/NetPrimer/AnalyzePrimer \\
\hline primers for &.$j \mathrm{sp}$ \\
\hline each gene & 11. Input copied forward and reverse primers, name of \\
\hline prior to & sequence and an optional description, and click "analyze" \\
\hline making & 12. Analyze "Rating" - Ideal rating is around $95 \%$ and higher \\
\hline selections to & for each primer, however not necessary if not possible for \\
\hline order. & selected gene \\
\hline 3.High & 13. Once a primer set with optimal ratings has been \\
\hline homology & determined, go to Primer-BLAST: \\
\hline with & http://www.ncbi.nlm.nih.gov/tools/primer-blast \\
\hline different & 14. Enter the selected forward and reverse primers into \\
\hline variants of & "Primer Parameters" box \\
\hline same gene is & 15. Check for homology with non-target genes, if found select \\
\hline desirable & $\begin{array}{l}\text { different primer set from PrimerQuest and repeat for each } \\
\text { gene }\end{array}$ \\
\hline
\end{tabular}




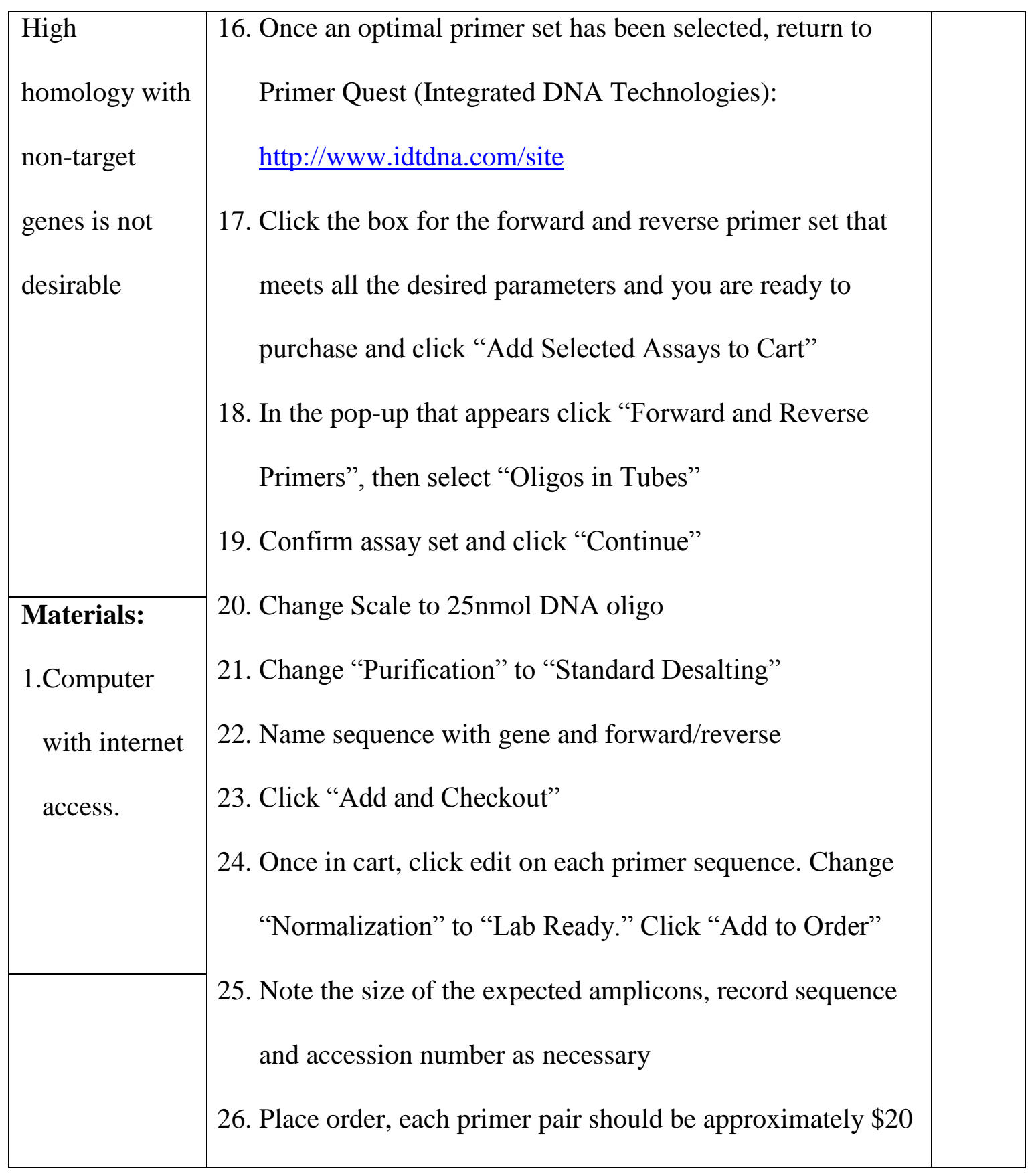

\begin{tabular}{|l|l|l|}
\hline \multicolumn{2}{|l|}{ Revision History } \\
\hline A & $\begin{array}{l}\text { Michael Gibbons } \\
\text { Sarah Ur }\end{array}$ & 140605 \\
\hline B & Tristin McGuffick & 150301 \\
\hline C & Shelby Kunz & 160601 \\
\hline
\end{tabular}




\section{APPENDIX H: Primer Sets Selected for Verification and Subsequent Amplification}

and Dissociation Curves

Table 8: Primer sets and characteristics for primer verification study.

\begin{tabular}{llccl}
\hline \multicolumn{1}{c}{ Primer ID } & Direction & Sequence (5' to 3') & $\begin{array}{c}\text { Length } \\
\text { (bp) }\end{array}$ & Homologs \\
\hline GAPDH 1 & Forward & CGACCACTTTGTCAAGCTCA & 20 & Self: 4 \\
(NM_001289745.1) & Reverse & AGGGGAGATTCAGTGTGGTG & 20 & Other: 10 \\
GAPDH 2 & Forward & GTATGACAACAGCCTCAAGAT & 21 & Self: 4 \\
(NM_001289745.1) & Reverse & TAGAGGCAGGGATGATGTT & 19 & Other: 0 \\
GAPDH 3 & Forward & GTCGGAGTCAACGGATTTG & 19 & Self: 3 \\
(NM_001289745.1) & Reverse & TCCATTGATGACAAGCTTCC & 20 & Other: 0 \\
BACT 1 & Forward & CCACCATGTACCCTGCATT & 20 & Self: 0 \\
(NM_001101.3) & Reverse & CGGACTCGTCATACTCCTGC & 20 & Other: 12 \\
BACT 2 & Forward & TGAGATTGGCATGGCTTTAT & 20 & Self: 1 \\
(NM_001101.3) & Reverse & CTTCCTGTAACAACGCATCT & 20 & Other: 19 \\
ENOS 1 & Forward & GTCCTGTGTATGGATGAGTATG & 22 & Self: 2 \\
(NM_000603.4) & Reverse & GCTGTTGAAGCGGATCTT & 18 & Other: 0 \\
ENOS 2 & Forward & TCCCTCGTGTGAAGAACT & 18 & Self: 8 \\
(NM_000603.4) & Reverse & GAGCTGTAGTGGTTGATG & 21 & Other: 4 \\
PECAM 1 & Forward & GCGGTGGGAAGATTACAAGA & 20 & Self: 1 \\
(NM_000442.4) & Reverse & TTTGGTGAGACCCACTTTCC & 20 & Other: 0 \\
PECAM 2 & Forward & CCAGCCCAGGATTTCTTATG & 20 & Self: 7 \\
(NM_000442.4) & Reverse & TCTGCAACACACTGGTATTC & 20 & Other: 0 \\
RAGE 1 & Forward & AGGAGACCAAGTCCAACTAC & 20 & Self: 10 \\
(NM_001136.4) & Reverse & CCTTCACAGATACTCCCTTCT & 21 & Other: 5 \\
RAGE 2 & Forward & AGTCCGTGTCTACCAGATT & 19 & Self: 10 \\
(NM_001136.4) & Reverse & GGTCTGTTCCTTCACAGATAC & 21 & Other: 1 \\
RAGE 3 & Forward & AGCGTGCAGAACTGAATC & 18 & Self: 8 \\
(NM_001136.4) & Reverse & GGCTCTGGTTGTAGAAGAAA & 20 & Other: 1 \\
\hline & & & &
\end{tabular}




\section{APPENDIX I: Preliminary Study RNA Assessment and Calculations}

Table 9: RNA isolation data from BVM study.

\begin{tabular}{|c|c|c|c|}
\hline Sample & A260/280 & $\begin{array}{l}\text { RNA Concentration } \\
(\mathrm{ng} / \mu \mathrm{L})\end{array}$ & RNA Volume $(\mu \mathrm{L})$ \\
\hline DHCAEC BVM 1 - & / & 0.4 & \\
\hline \multirow[t]{2}{*}{ Normal Glucose Media } & 1.57 & 1.5 & \\
\hline & 1.79 & 1.7 & Insufficient RNA \\
\hline DHCAEC BVM 2 - & 1.67 & 14.1 & \\
\hline \multirow[t]{2}{*}{ Normal Glucose Media } & 1.71 & 11.7 & \\
\hline & 1.29 & 20.2 & 13.04 \\
\hline DHCAEC BVM 3 - & 2.03 & 17.1 & \\
\hline \multirow{2}{*}{ Normal Glucose Media } & 1.67 & 28.7 & \\
\hline & 1.87 & 17.6 & 9.46 \\
\hline DHCAEC BVM 4 - & 1.97 & 31.1 & \\
\hline \multirow[t]{2}{*}{ High Glucose Media } & 1.96 & 31.3 & \\
\hline & 1.93 & 32.3 & 6.34 \\
\hline DHCAEC BVM 5 - & 1.13 & 69.4 & \\
\hline \multirow[t]{2}{*}{ High Glucose Media } & 1.52 & 10.3 & \\
\hline & 1.56 & 6.5 & 12.22 \\
\hline DHCAEC BVM 6 - & 2.22 & 13.8 & \\
\hline \multirow{2}{*}{ High Glucose Media } & 1.75 & 21.0 & \\
\hline & 1.65 & 22.9 & 10.39 \\
\hline HUVEC BVM 1 - & 2.26 & 16.0 & \\
\hline \multirow[t]{2}{*}{ Normal Glucose Media } & 1.95 & 22.8 & \\
\hline & 2.06 & 15.9 & 10.97 \\
\hline HUVEC BVM 2 - & 2.12 & 43.3 & \\
\hline \multirow[t]{2}{*}{ Normal Glucose Media } & 2.07 & 46.8 & \\
\hline & 2.06 & 46.2 & 4.40 \\
\hline HUVEC BVM 3 - & 2.16 & 22.9 & \\
\hline \multirow[t]{2}{*}{ High Glucose Media } & 2.16 & 21.6 & \\
\hline & 2.05 & 21.6 & 9.08 \\
\hline HUVEC BVM 4 - & 2.44 & 18.3 & \\
\hline \multirow[t]{2}{*}{ High Glucose Media } & 2.15 & 20.0 & \\
\hline & 2.29 & 19.6 & 10.36 \\
\hline HUVEC BVM 5 - & 1.53 & 20.9 & \\
\hline \multirow[t]{2}{*}{ High Glucose Media } & l & l & \\
\hline & l & l & 9.57 \\
\hline HUVEC BVM 6 - & 2.21 & 20.6 & \\
\hline \multirow{2}{*}{ High Glucose Media } & 2.13 & 20.6 & \\
\hline & 2.29 & 19.9 & 9.82 \\
\hline
\end{tabular}




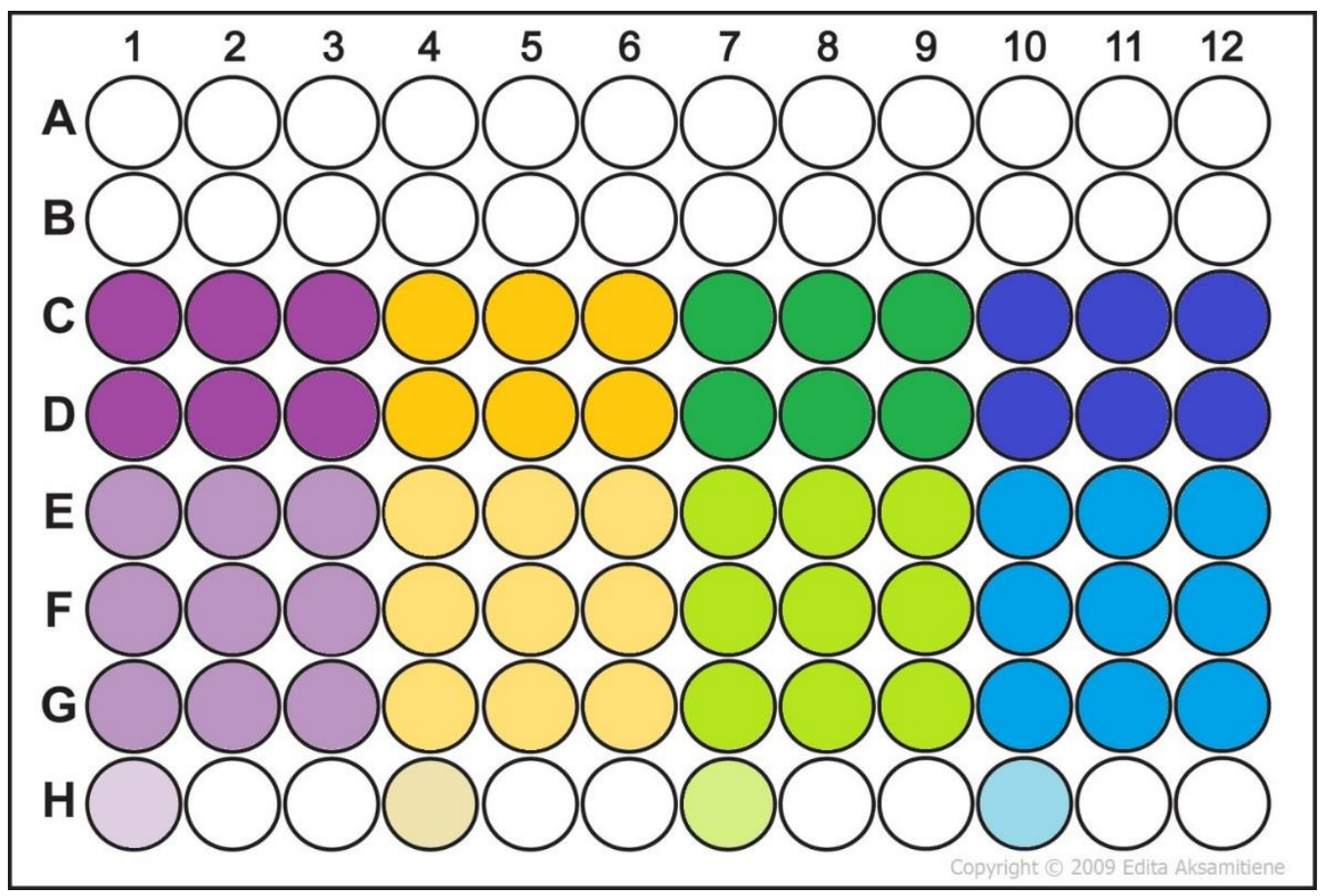

Figure 45: First plate scheme for experiment in chapter 4, where each color represents a gene: GAPDH, PECAM, RAGE, and $e N O S$; rows $\mathrm{C}$ and $\mathrm{D}$ are RNA samples isolated from BVMs composed of DHCAECs and cultured in normal glucose media; rows E-G are RNA samples isolated from BVMs composed of DHCAECs and cultured in high glucose media; and row $\mathrm{H}$ are non-template controls. 


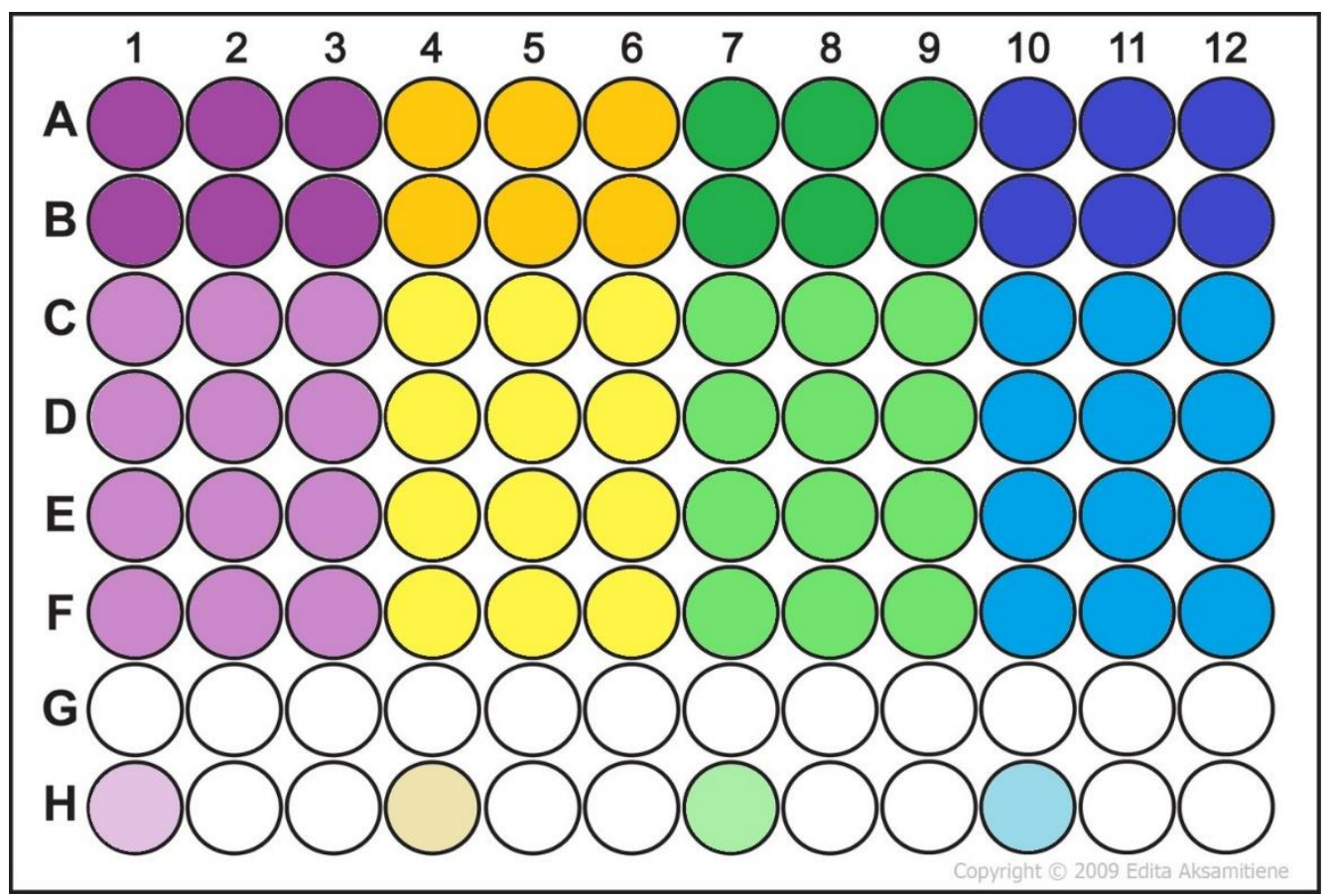

Figure 46: Second plate scheme for experiment in chapter 4, where each color represents a gene: GAPDH, PECAM, RAGE, and $e N O S$; rows A and $\mathrm{B}$ are RNA samples from BVMs composed of HUVECs and cultured in normal glucose media; rows $\mathrm{C}-\mathrm{F}$ are RNA samples from BVMs composed of HUVECs and cultured in high glucose media; and row are non-template controls. 


\section{APPENDIX K: Data and Minitab Statistical Analysis from Preliminary Study}

Comparing Glucose Concentrations

PECAM

\section{Two-Sample T-Test and CI}

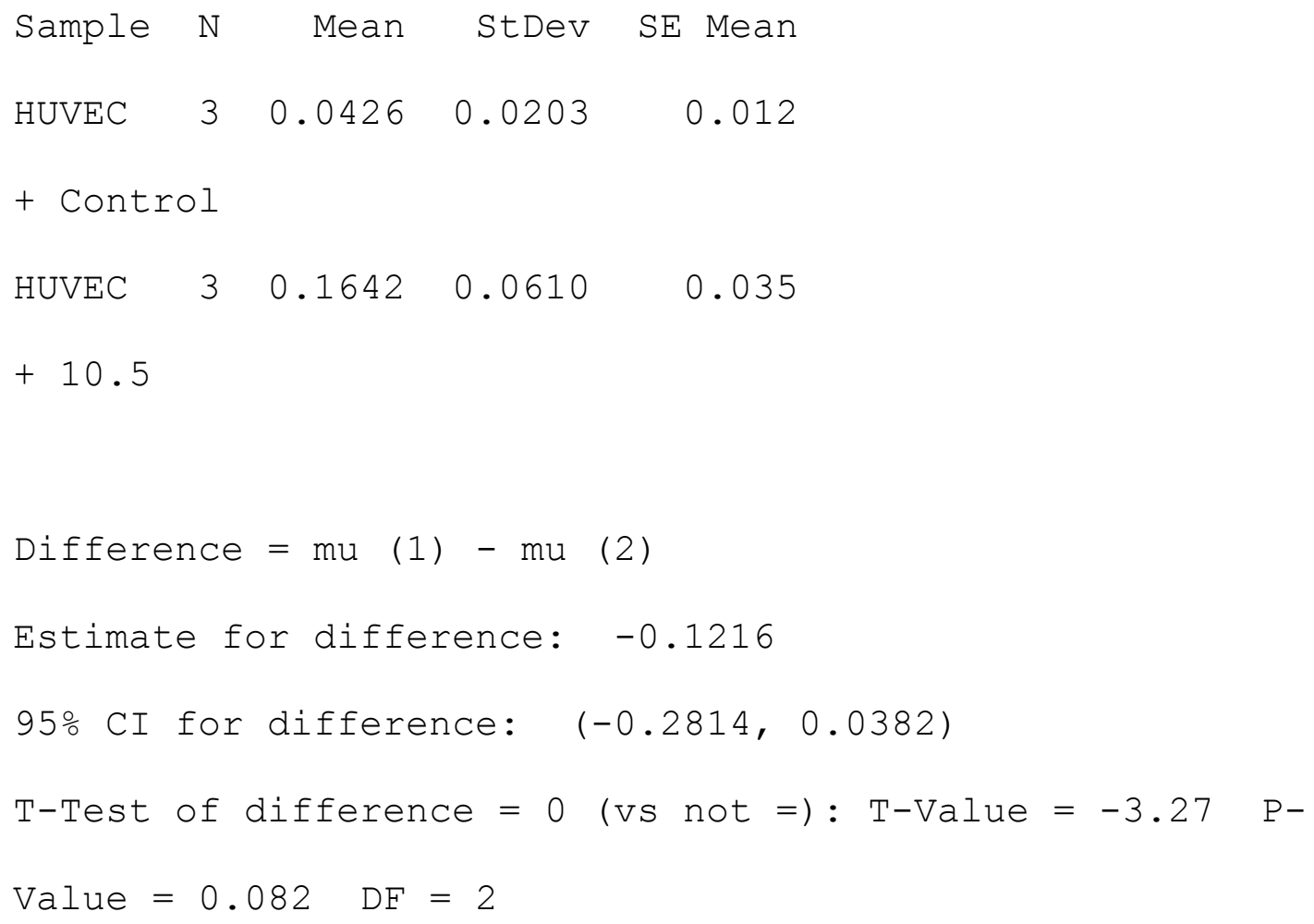

\section{Two-Sample T-Test and CI}

$\begin{array}{lllll}\text { Sample } & N & \text { Mean } & \text { StDev } & \text { SE Mean } \\ \text { HUVEC } & 3 & 0.171 & 0.114 & 0.066 \\ +\quad 25.5 & & & & \end{array}$




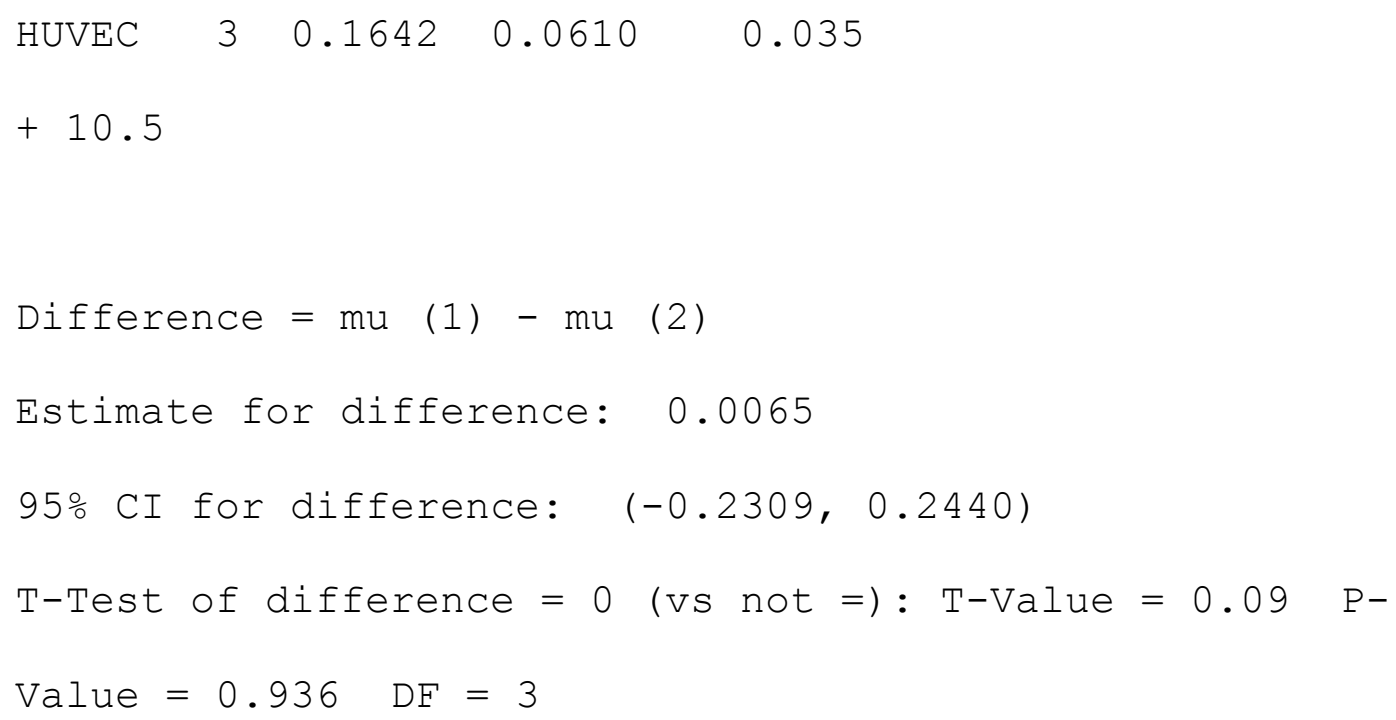

\section{Two-Sample T-Test and CI}

$\begin{array}{lrrrr}\text { Sample N } & \text { Mean } & \text { StDev } & \text { SE Mean } \\ \text { HUVEC } & 3 & 0.171 & 0.114 & 0.066 \\ +25.5 & & & & \\ \text { HUVEC 3 } & 0.0426 & 0.0203 & 0.012 \\ \text { + Control } & & & \end{array}$

Difference $=\operatorname{mu}(1)-\operatorname{mu}(2)$

Estimate for difference: 0.1281

95\% CI for difference: (-0.1593, 0.4155)

T-Test of difference $=0$ (vs not $=)$ : T-Value $=1.92 \mathrm{P}-$ Value $=0.195 \quad \mathrm{DF}=2$

\section{Two-Sample T-Test and CI}




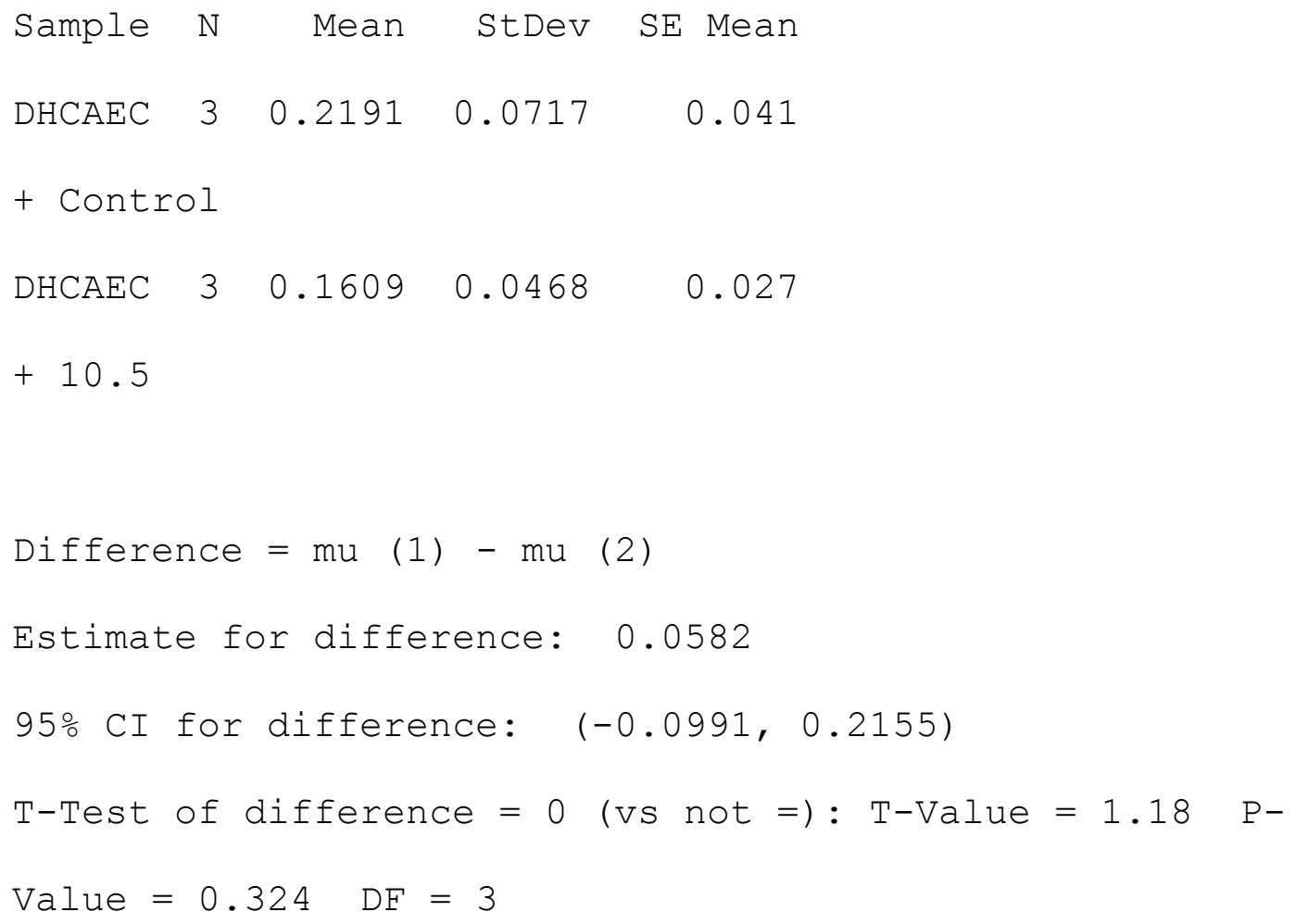

\section{Two-Sample T-Test and CI}

$\begin{array}{lrrrr}\text { Sample N } & \text { Mean } & \text { StDev } & \text { SE Mean } \\ \text { DHCAEC 3 } & 0.2191 & 0.0717 & 0.041 \\ + \text { Control } & & & \\ \text { DHCAEC 3 } & 0.319 & 0.279 & 0.16 \\ +25.5 & & & & \end{array}$

Difference $=\mathrm{mu}(1)-\mathrm{mu}$ (2)

Estimate for difference: $\quad-0.100$

95 CI for difference: (-0.815, 0.614) 
T-Test of difference $=0$ (vs not $=$ ) : T-Value $=-0.60 \mathrm{P}-$ Value $=0.607 \quad \mathrm{DF}=2$

\section{Two-Sample T-Test and CI}

Sample N Mean StDev SE Mean

$\begin{array}{llll}\text { DHCAEC } 3 & 0.1609 & 0.0468 & 0.027\end{array}$

$+10.5$

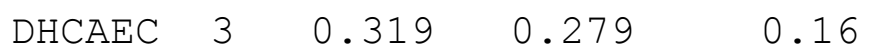

$+25.5$

Difference $=$ mu (1) $-\mathrm{mu}$ (2)

Estimate for difference: $\quad-0.159$

95\% CI for difference: $(-0.860,0.543)$

T-Test of difference $=0$ (vs not $=$ ) : T-Value $=-0.97$ P-

Value $=0.433 \quad \mathrm{DF}=2$

\section{$R A G E$}

\section{Two-Sample T-Test and CI}

$\begin{array}{rrrrr}\text { Sample } & \text { N } & \text { Mean } & \text { StDev } & \text { SE Mean } \\ \mathrm{H}+\mathrm{C} & 3 & 0.000725 & 0.000306 & 0.00018 \\ \mathrm{H}+10.5 & 3 & 0.00266 & 0.00112 & 0.00065\end{array}$




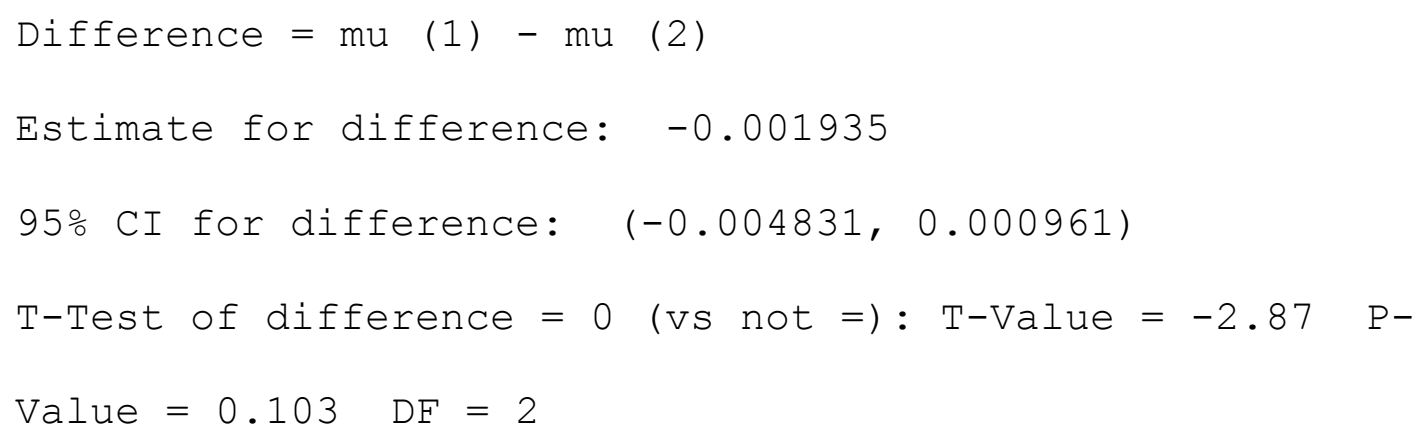

\section{Two-Sample T-Test and CI}

$\begin{array}{rrrrr}\text { Sample } & \text { N } & \text { Mean } & \text { StDev } & \text { SE Mean } \\ \mathrm{H}+\mathrm{C} & 3 & 0.000725 & 0.000306 & 0.00018 \\ \mathrm{H}+25.5 & 3 & 0.00190 & 0.00183 & 0.0011\end{array}$

Difference $=$ mu (1) - mu (2)

Estimate for difference: $\quad-0.00117$

95 CI for difference: (-0.00578, 0.00343)

T-Test of difference $=0$ (vs not $=$ ) : T-Value $=-1.10$ PValue $=0.387 \quad \mathrm{DF}=2$

\section{Two-Sample T-Test and CI}

$\begin{array}{rrrrr}\text { Sample } & \text { N } & \text { Mean } & \text { StDev } & \text { SE Mean } \\ \text { H+10.5 } & 3 & 0.00266 & 0.00112 & 0.00065 \\ \text { H+25.5 } & 3 & 0.00190 & 0.00183 & 0.0011\end{array}$




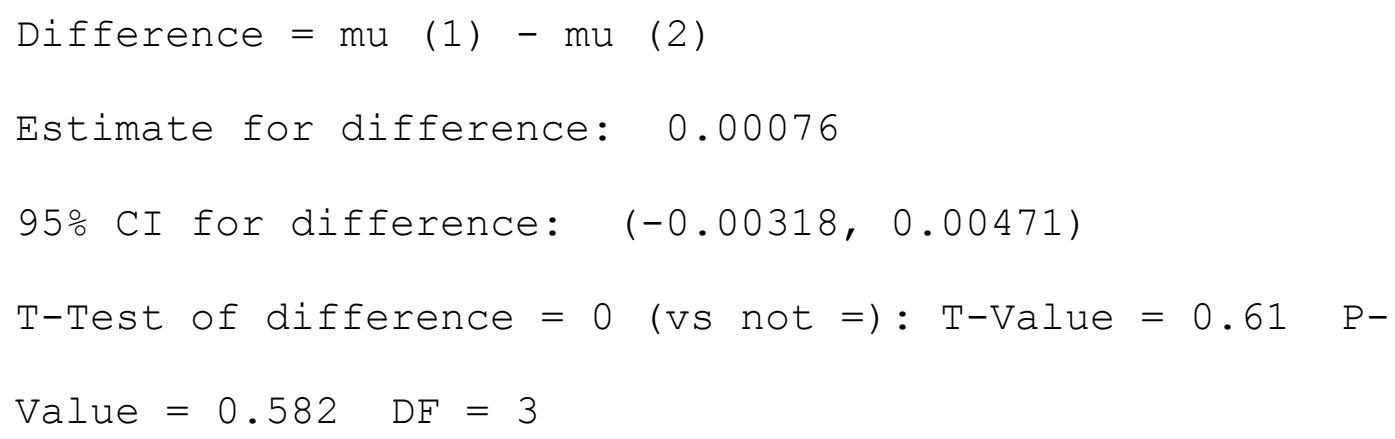

\section{Two-Sample T-Test and CI}

$\begin{array}{rrrrr}\text { Sample } & \mathrm{N} & \text { Mean } & \text { StDev } & \text { SE Mean } \\ \text { D + C } & 3 & 0.0002740 & 0.0000960 & 0.000055 \\ \text { D+10.5 } & 3 & 0.000909 & 0.000319 & 0.00018\end{array}$

Difference $=$ mu (1) - mu (2)

Estimate for difference: $\quad-0.000635$

95\% CI for difference: (-0.001462, 0.000192$)$

T-Test of difference $=0$ (vs not $=$ ) : T-Value $=-3.30 \mathrm{P}-$ Value $=0.081 \quad \mathrm{DF}=2$

\section{Two-Sample T-Test and CI}

$\begin{array}{rrrrr}\text { Sample } & \text { N } & \text { Mean } & \text { StDev } & \text { SE Mean } \\ \text { D }+ \text { C } & 3 & 0.0002740 & 0.0000960 & 0.000055 \\ \text { D+25.5 } & 3 & 0.000753 & 0.000209 & 0.00012\end{array}$




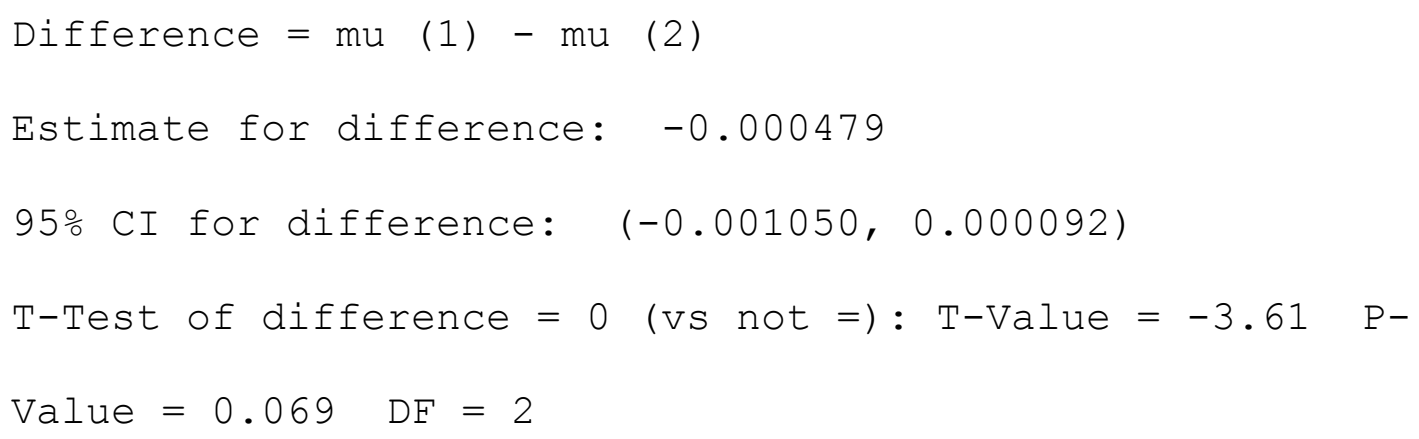

\section{Two-Sample T-Test and CI}

$\begin{array}{rrrrrr}\text { Sample } & \text { N } & \text { Mean } & \text { StDev } & \text { SE Mean } \\ \text { D+10.5 } & 3 & 0.000909 & 0.000319 & 0.00018 \\ \text { D+25.5 } & 3 & 0.000753 & 0.000209 & 0.00012\end{array}$

Difference $=\operatorname{mu}(1)-\operatorname{mu}(2)$

Estimate for difference: 0.000155

95\% CI for difference: (-0.000545, 0.000856)

T-Test of difference $=0($ vs not $=)$ : T-Value $=0.71 \mathrm{P}-$ Value $=0.531 \quad \mathrm{DF}=3$

eNOS

\section{Two-Sample T-Test and CI}

$\begin{array}{rrrrr}\text { Sample } & \text { N } & \text { Mean } & \text { StDev } & \text { SE Mean } \\ \text { H }+ \text { C } & 3 & 0.000698 & 0.000486 & 0.00028 \\ \text { H+10.5 } & 3 & 0.01349 & 0.00206 & 0.0012\end{array}$




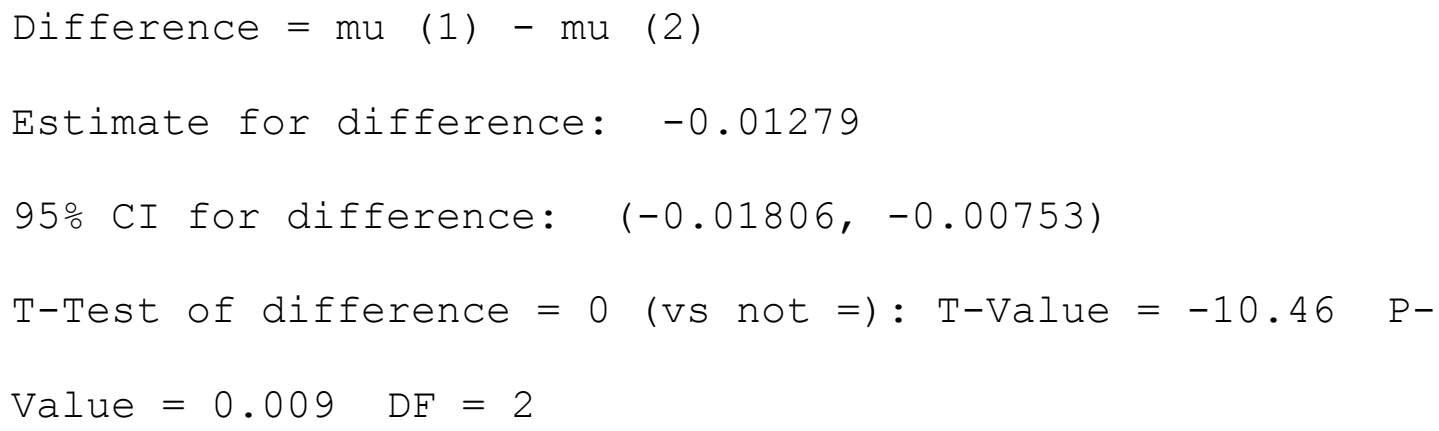

\section{Two-Sample T-Test and CI}

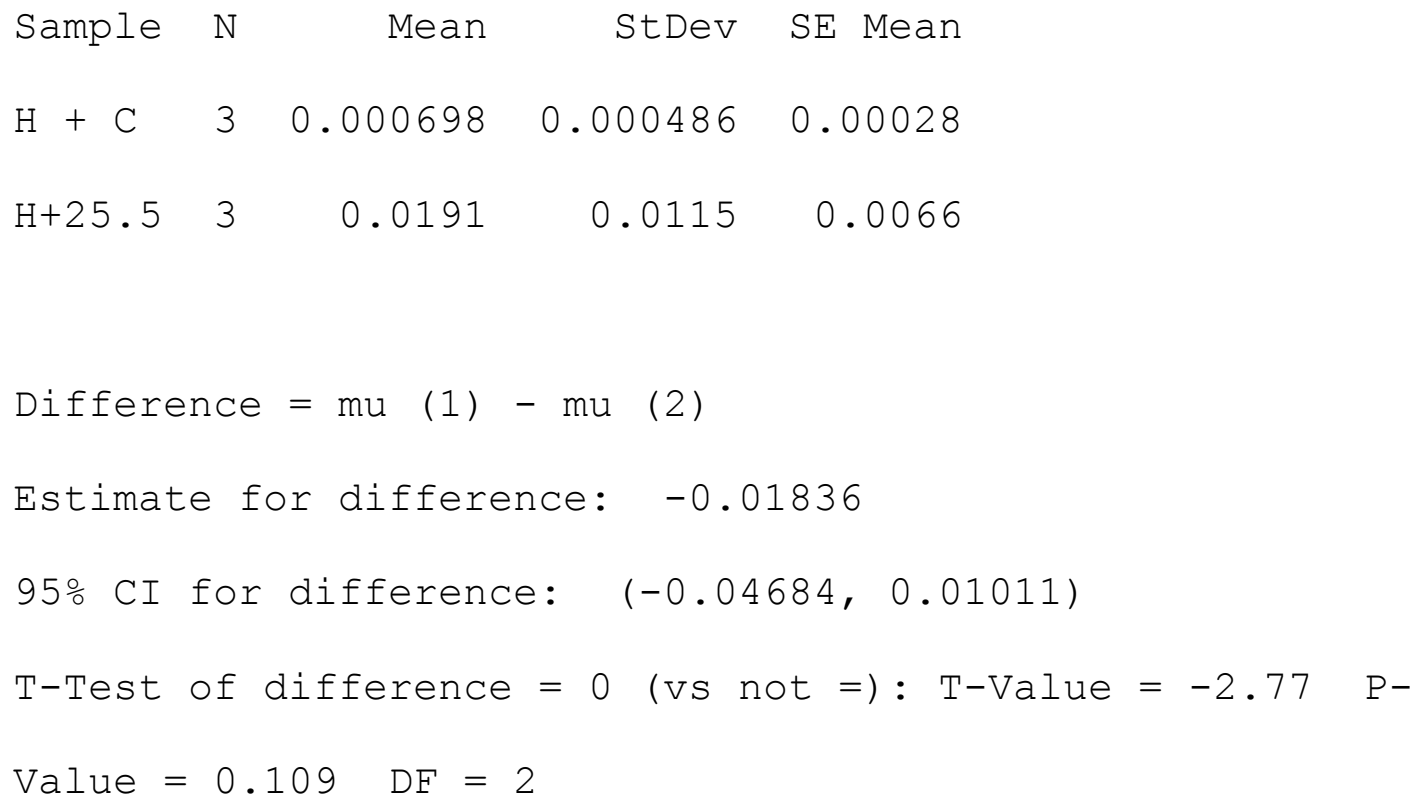

\section{Two-Sample T-Test and CI}

$\begin{array}{rrrrr}\text { Sample N } & \text { Mean } & \text { StDev } & \text { SE Mean } \\ \text { H+10.5 } & 3 & 0.01349 & 0.00206 & 0.0012 \\ \text { H+25.50 } & 3 & 0.0191 & 0.0115 & 0.0066\end{array}$




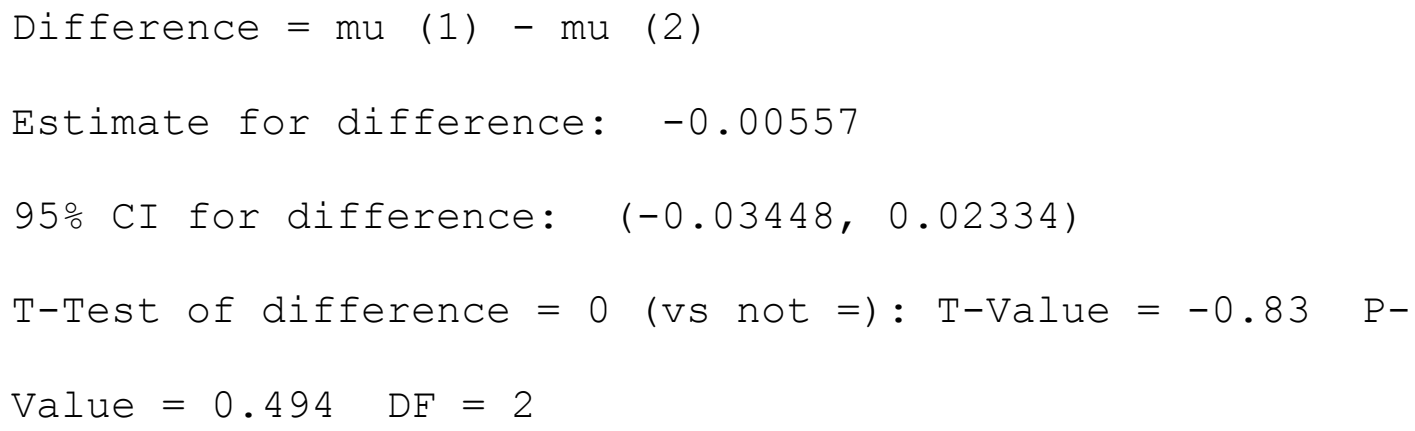

\section{Two-Sample T-Test and CI}

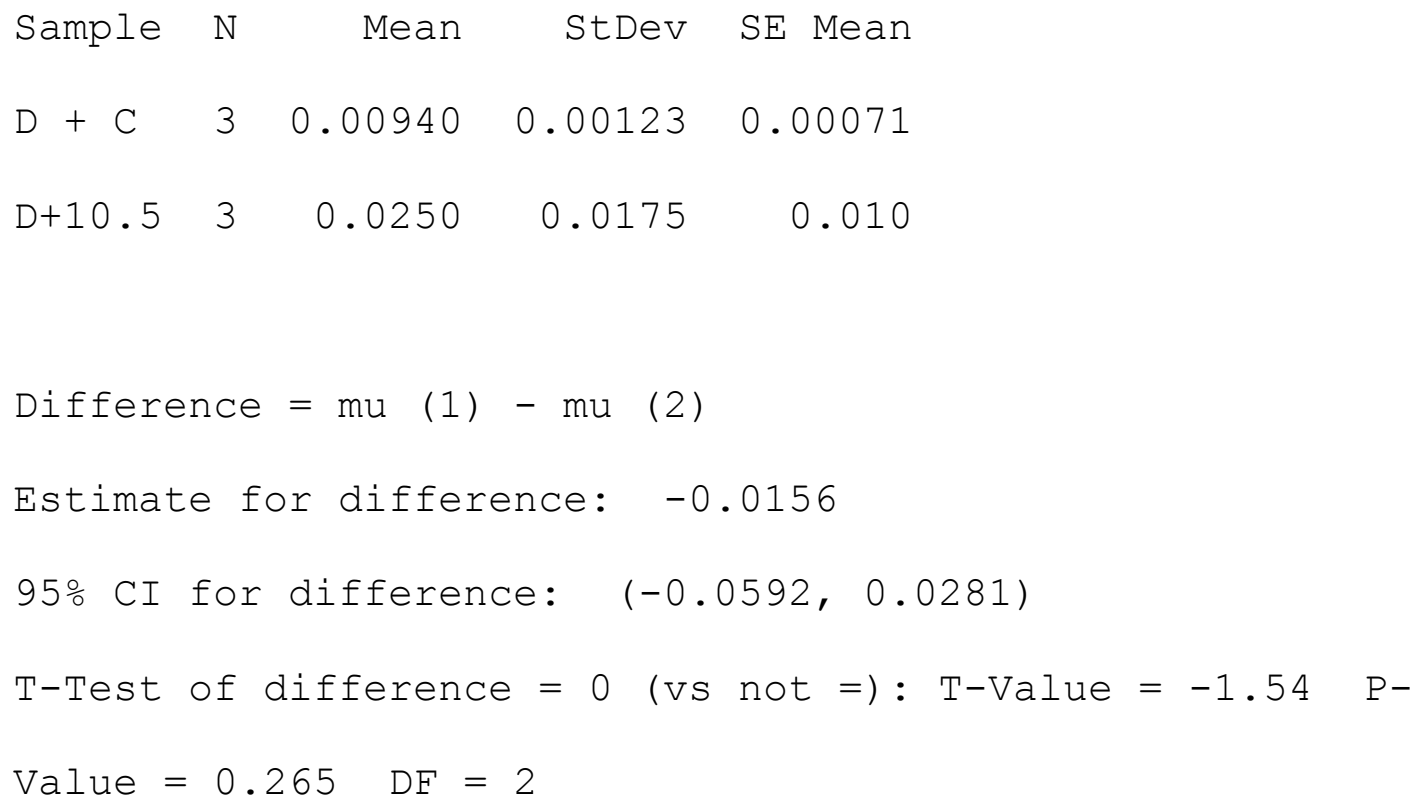

\section{Two-Sample T-Test and CI}

$\begin{array}{rrrrr}\text { Sample } & \text { N } & \text { Mean } & \text { StDev } & \text { SE Mean } \\ \text { D + C } & 3 & 0.00940 & 0.00123 & 0.00071 \\ \text { D+25.5 } & 3 & 0.007278 & 0.000571 & 0.00033\end{array}$




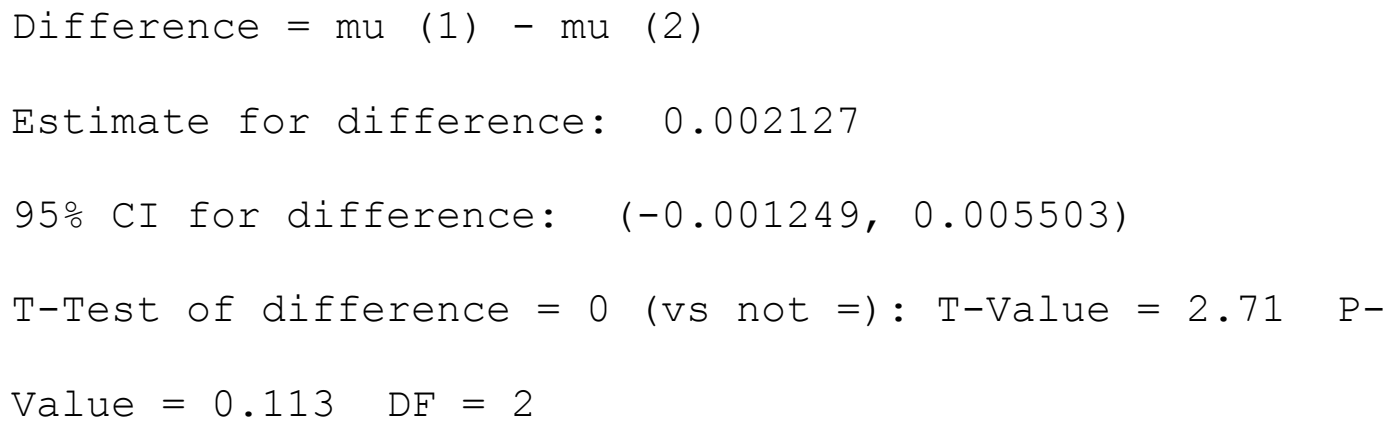

\section{Two-Sample T-Test and CI}

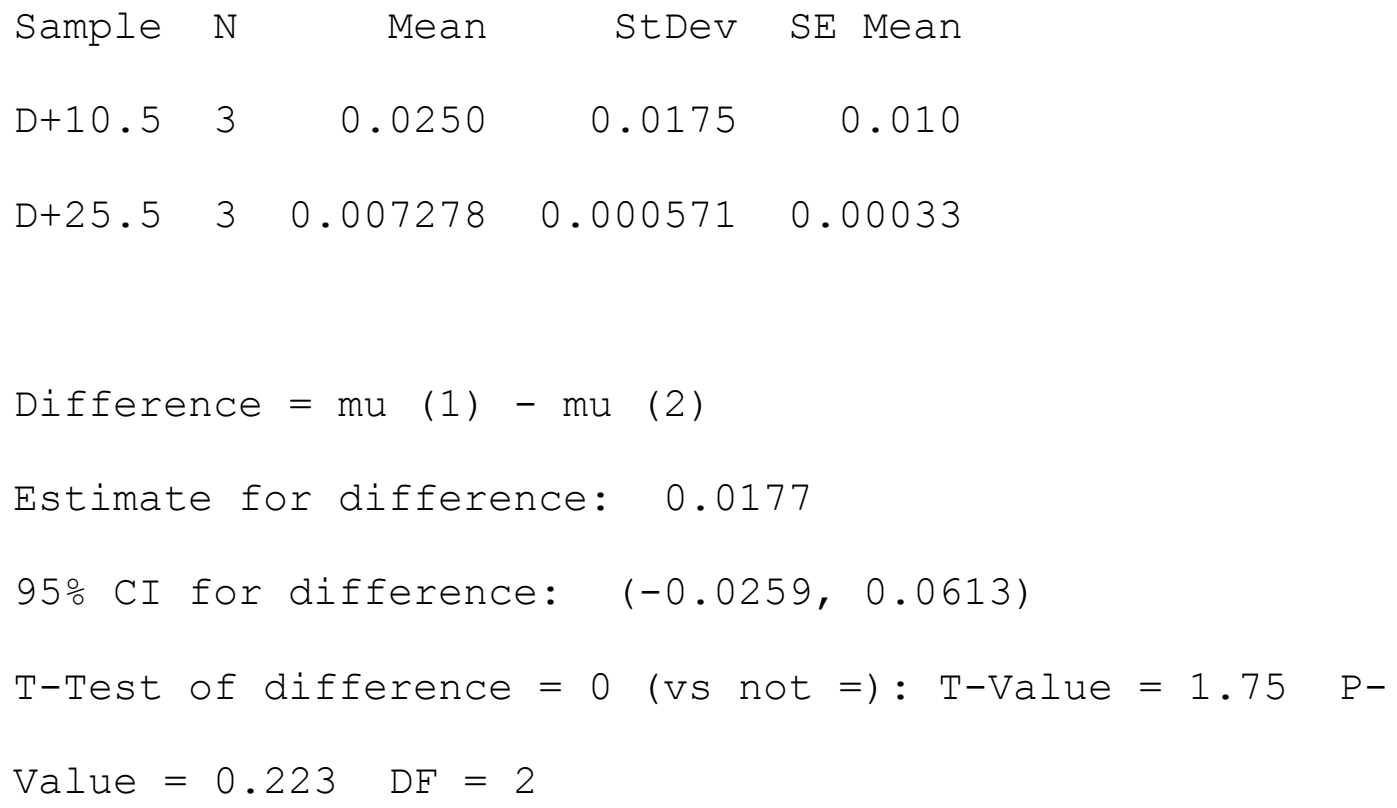




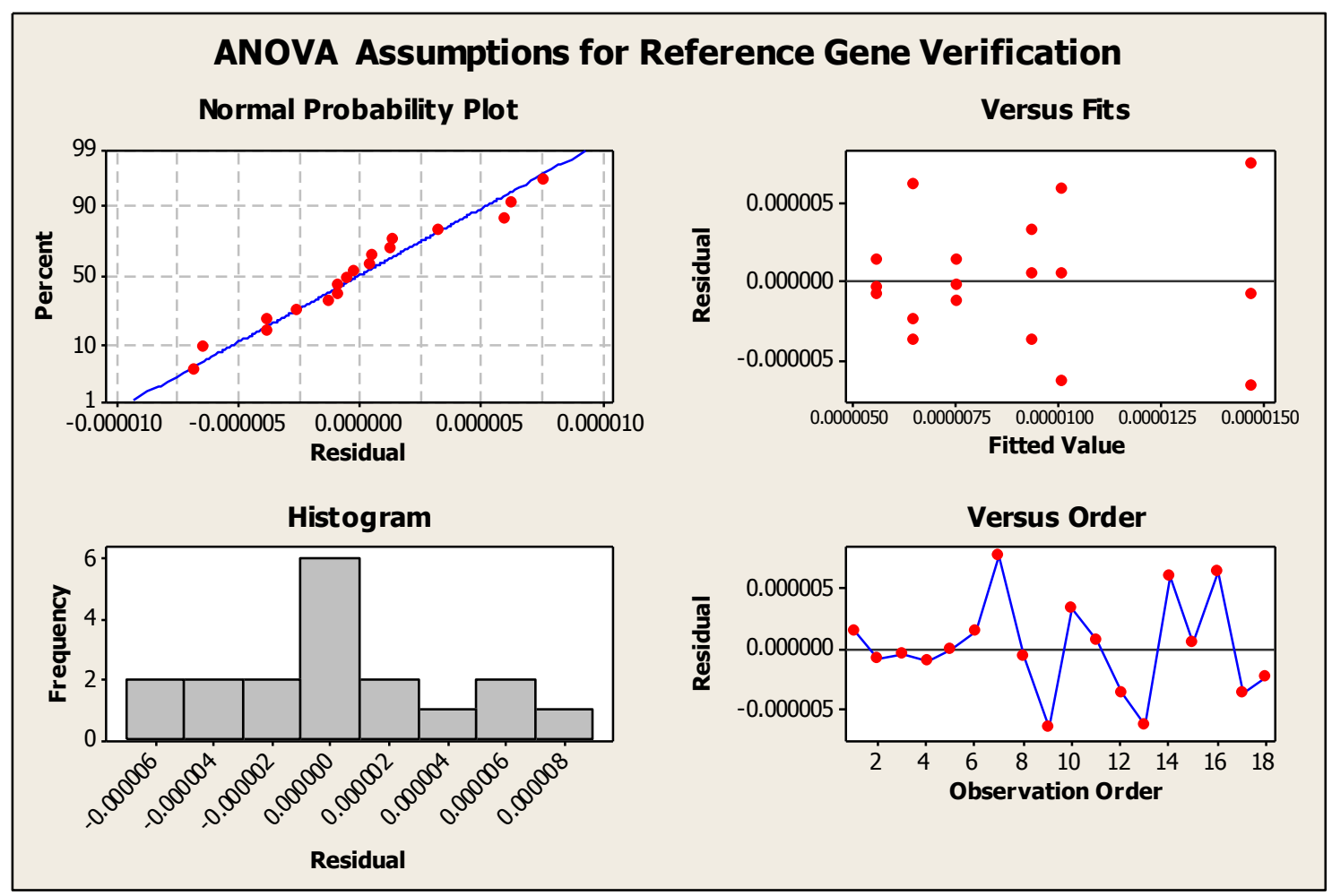

